\title{
MINERAL PRODUCTION IN IOWA FOR THE YEARS 1933-1938
}

by

H. GARLAND HERSHEY 
. 


\section{CONTENTS}

PAGE

FORE,WORD

INTRODUCTION

ACKNOWLEDGMENTS -

General STATEMENT

CEMENT -

CLAY -

COAL _.

GYPSUM

LIMESTONE _.

SAND AND GRAVEL_- 



\section{MINERAL PRODUCTION IN IOWA FOR THE YEARS 1933-1938}

\section{Foreword}

This report on the mineral production of Iowa from 1933 to 1938 continues the series of statistical summaries published by the lowa Geological Survey. It is the first such report since the statistics for 1932 were published and has been somewhat delayed because of various changes in personnel and alterations in policy of the Survey to meet increasing public demands for service and information.

The primary function of these reports is to provide in convenient form an accurate record of economic developments and trends in the mineral industries of Iowa for the information and use of the producers and the public.

The form of previous reports is followed as closely as possible in order to facilitate comparison with earlier statistics and, as in the past, the figures on the last year reported (1932) are repeated for comparative purposes. It was found necessary, however, to make certain changes in the presentation of data, which are discussed under their proper headings.

\section{Introduction}

All minerals produced in Iowa during the period 1933-1938 were of the nonmetallic type. Of these, coal was the most valuable. It had an average annual value of approximately 8.6 millions of dollars and was followed by cement which averaged about 7.1 millions of dollars for 1936-1938. These two maintained their respective ranks throughout the period of this report. Limestone, gypsum, clay products, and sand and gravel, the other leading mineral products, changed ranking positions almost yearly.

From a national viewpoint gypsum is the most important Iowa mineral produced. In 1935 approximately 14 per cent of the total production of the United States was from Iowa, which again placed her third in rank by production and value, superceded only by New 
York and Michigan. Normally these three leading states yield slightly more than 50 per cent of the total domestic output.

In addition to the minerals already mentioned, Iowa produced small amounts of sandstone, peat, and stone listed as miscellaneous. There were few producers of any one of these and, to avoid revealing confidental figures, no individual statistics are presented for them.

No data are available on wool rock utilized by the one plant in the state which began operations in 1937. It is known, however, that only a small amount of raw material from Iowa is utilized.

Lists of operators in each industry except coal are included under the discussion of individual minerals. These lists are the most recent available and include operators producing in 1939, although some nonproducers are also included. They do not include all operators producing during the period of this report. No up-to-date list of coal producers was available when this report went to press.

\section{Acknowledgments}

Statistics on the production of most minerals are collected by the United States Bureau of Mines cooperating with the various state geological surveys. Each year the Bureau of Mines publishes the results of their canvasses in a Minerals Yearbook. Most of the data appearing in the report on mineral production in Iowa from 1933 to 1938 were obtained through the cooperative agreement between the Bureau of Mines and the Iowa Geological Survey and from the Minerals Yearbooks covering the period.

The canvass for clay products is made by the United States Bureau of Census and statistics for this industry appearing in the following report were furnished by the Bureau of Census.

The collection of production statistics of the bituminous-coal industry previously conducted by the Bureau of Mines was relinquished on July 1, 1937 to the National Bituminous Coal Commission which has contributed the chapter on bituminous coal to the subsequent Minerals Yearbooks. Statistics on coal for this report for 1936-1938 are taken entirely from the Minerals Yearbooks.

In addition to acknowledging the cooperation of the Federal agencies it is a pleasure to express appreciation to the various mineral producers, particularly in Iowa, for their reports which make the following statistics available.

Tabulation of production figures for this period - begun by $\mathrm{Dr}$. 
A. C. Tester in 1934 - was taken over in 1937 by the writer who is responsible for the statements to follow. The tables presenting sand and gravel data by counties were prepared by Mr. Walter C. Schuldt, similar tables on limestone were prepared by Mr. Maxim M. Elias, and Miss Kathryn T. Neuzil assisted in checking all figures.

\section{General Statement}

The years 1933-1938 represent a particularly interesting periodi in the history of mineral production in that they include the latter part of the depression which began in 1929, the recovery period from 1934 to early 1937 and a so-called recession which was believed ended in the last half of 1938 .

The year 1934 was a turning point for mineral industries in Iowa. From 1929 until 1933 the total value of mineral products had decreased yearly 'and in 1933 was $\$ 15,154,652$, the lowest since 1905. In 1934 an upswing started which resulted in increases each succeeding year including 1937. The recession began too late in 1937 to affect greatly the mineral industries in that year, but its effect was felt in 1938 when there was a decline in total value. Iowa, however, did not suffer as greatly during this period as did the United States as a whole. Conditions improved during the last half of 1938 and at the end of the year the outlook was favorable.

The quantity and value of mineral products in Iowa from 1932 to 1938 are showh in Table I. It should be noted that in 1937 and 1938 a new method of reporting the value of gypsum was instituted which makes it appear that the value dropped sharply in 1937, when as a matter of fact the value of gypsum in Iowa increased in that year (see explanation under Gypsum). This should also be remembered when comparing total state values for 1937 and 1938 with those of earlier years.

Mineral production in the United States which had shown a decrease in value each year since 1929 began an upward trend in 1933. The total value increased yearly until 1937 when it was greater than for 1928 and only slightly less than 1929. Metallics were particularly strong during the 1933-37 period and increased in value each year while the nonmetallics showed an increase each year except for the fuels in 1933 and other nonmetals in 1934. In 1938 the value of both metallics and nonmetallics dropped off sharply because of the recession. In spite of declines in production during late 1937 and early 1938, 
TABLE I

Mineral Production in Iowa from 1932 to 1938

\begin{tabular}{|c|c|c|c|c|c|c|c|c|}
\hline & Cement & Clay $^{\mathrm{a}}$ & Coal & Gypsum & Limestone & $\begin{array}{c}\text { Sand and } \\
\text { gravel }\end{array}$ & Miscellaneous & Total value ${ }^{b}$ \\
\hline $\begin{array}{l}1932 \\
\text { Unit } \\
\text { Quantity } \\
\text { Value } \\
\text { Producers } \\
1933\end{array}$ & $\begin{array}{r}\text { bbls. } \\
4,373,642 \\
\$ 3,907,427 \\
5\end{array}$ & 805,799 & $\begin{array}{r}\text { tons } \\
3,862,435 \\
9,254,000 \\
212\end{array}$ & $\begin{array}{r}\text { tons } \\
178,087 \\
1,468,414 \\
7\end{array}$ & $\begin{array}{c}\text { tons } \\
1,591,240^{\mathrm{c}} \\
1,389,465 \\
-53\end{array}$ & $\begin{array}{r}5,230,562 \\
1,706,874 \\
87 \mathrm{e}\end{array}$ & $387,064 d$ & $18,522,625$ \\
\hline $\begin{array}{l}\text { Quantity } \\
\text { Value } \\
\text { Producers } \\
1934\end{array}$ & $\begin{array}{r}2,770,656 \\
\$ 3,651,921 \\
5\end{array}$ & 917,548 & $\begin{array}{r}3,194,983 \\
7,217,000 \\
242\end{array}$ & $\begin{array}{r}172,555 \\
1,357,407 \\
8\end{array}$ & $\begin{array}{r}1,050,190^{t} \\
920,532 \\
60\end{array}$ & $\begin{array}{r}4,343,781 \\
1,165,066 \\
60\end{array}$ & $900,203^{d}$ & $15,154,652$ \\
\hline $\begin{array}{l}\text { Quantity } \\
\text { Value } \\
\text { Producers }\end{array}$ & $\begin{array}{r}3,340,049 \\
\$ 5,094,922 \\
5\end{array}$ & $1,374,469$ & $\begin{array}{l}3,366,992 \\
7,862,000 \\
243 \mathrm{~g}\end{array}$ & $\begin{array}{r}180,271 \\
1,670,356 \\
8\end{array}$ & $\begin{array}{c}2,276,443^{\circ} \\
1,934,364 \\
101\end{array}$ & $\begin{array}{c}4,349,362^{\mathrm{c}} \\
1,394,000^{\mathrm{c}} \\
45\end{array}$ & $1,320,509^{d, ~} \mathbf{h}$ & $19,326,181$ \\
\hline $\begin{array}{l}1935 \\
\text { Quantity } \\
\text { Value } \\
\text { Producers } \\
1936\end{array}$ & $\begin{array}{r}3,203,301 \\
\$ 5,072,098 \\
5\end{array}$ & $2,039,568$ & $\begin{array}{r}3,650,163 \\
9,002,000 \\
263 \mathrm{~g}\end{array}$ & $\begin{array}{r}230,203 \\
2,215,770 \\
7\end{array}$ & $\begin{array}{r}1,840,080 \\
1,645,937 \\
105\end{array}$ & $\begin{array}{r}5,732,742 \\
1,756,851 \\
49\end{array}$ & $1,714,363^{\mathrm{d}, \mathrm{h}}$ & $21,709,817$ \\
\hline $\begin{array}{l}\text { Quantity } \\
\text { Value } \\
\text { Producers } \\
1937\end{array}$ & $\begin{array}{r}4,407,624 \\
\$ 6,908,225 \\
5\end{array}$ & $2,774,833$ & $\begin{array}{r}3,960,700^{1} \\
9,940,000^{1} \\
3618\end{array}$ & $\begin{array}{r}344,221 \mathrm{~J} \\
3,261,388 \mathrm{~J} \\
8\end{array}$ & $\begin{array}{r}4,003,550 \\
3,397,356 \\
130\end{array}$ & $\begin{array}{r}6,293,984 \\
2,048,282 \\
51\end{array}$ & $1,762,575^{d, ~ h ~}$ & $28,359,140$ \\
\hline $\begin{array}{l}\text { Quantity } \\
\text { Value } \\
\text { Producers } \\
1938\end{array}$ & $\begin{array}{r}4,598,453 \\
\$ 7,046,021 \\
5\end{array}$ & $3,301,548$ & $\begin{array}{c}3,637,0541 \\
9,529,000^{1, \mathrm{~m}} \\
340 \mathrm{~g}\end{array}$ & $\begin{array}{c}387,255^{\mathrm{k}} \\
533,162^{\mathrm{k}} \\
8\end{array}$ & $\begin{array}{r}4,294,310^{1} \\
4,276,891 \\
174\end{array}$ & $\begin{array}{r}6,397,154 \\
2,235,103 \\
107 \text { e }\end{array}$ & $2,163,370^{d, b}$ & $26,941,350^{n_{r}-p}$ \\
\hline $\begin{array}{l}\text { Quantity } \\
\text { Value } \\
\text { Producers }\end{array}$ & $\begin{array}{r}4,759,390 \\
\$ 7,327,048 \\
5\end{array}$ & $2,913,992$ & $\begin{array}{r}3,250,000^{\mathrm{m}} \\
8,340,000^{\mathrm{m}} \\
300^{\mathrm{m}}\end{array}$ & $\begin{array}{r}364,920 \\
495,856 \\
8\end{array}$ & $\begin{array}{c}3,369,7501 \\
3,782,480 \\
85\end{array}$ & $\begin{array}{r}6,994,286^{c} \\
2,299,732^{c} \\
107 \mathrm{e}\end{array}$ & & $25,159,058^{n, p}$ \\
\hline
\end{tabular}

b With duplications eliminated. Value of

d Includes ferro-alloys and pig iron, value not included in total value for state.

Includes noncommercial producers.

I Includes sandstone.

Includes peat.

Gypsum mined; value as sold (crude and calcined) comparable value for 1937 not ayailable

$k$ Gypsum mined; value of crude at mine as reported by producers. Comparable value for earlier years not available.

m Estimated.

in

Note change in method of reporting gypsum.
Total value for $1937-1938$ not comparable with earlier years. 
the mineral industries enjoyed better conditions than did business in general. Table II shows statistically the mineral production in the United States for the period of this report.

TABLE II

Mineral Production in the United States from 1932 to 1938

\begin{tabular}{|c|c|c|c|c|c|}
\hline \multirow[b]{2}{*}{ Year } & \multirow[b]{2}{*}{ Metallic } & \multicolumn{3}{|c|}{ Nonmetallic } & \multirow[b]{2}{*}{ Grand Total } \\
\hline & & Fuels ${ }^{1}$ & Other & Total & \\
\hline 1932 & $\$ 285,875,000$ & $\$ 1,743,400,000$ & $\$ 432,425,000$ & $\$ 2,175,825,000$ & $\$ 2,461,700,000$ \\
\hline 1933 & $417,065,000$ & $1,683,400,000$ & $454,635,000$ & $2,138,035,000$ & $2,555,100,000$ \\
\hline 1934 & $548,934,000$ & $2,233,300,000$ & $543,166,000$ & $2,776,466,000$ & $3,325,400,000$ \\
\hline 1935 & $733,130,000$ & $2,330,000,000$ & $586,870,000$ & $2,916,870,000$ & $3,650,000,000$ \\
\hline 1936 & $1,081,600,000$ & $2,759,200,000$ & $716,000,000$. & $3,475,200,000$ & $4,556,800,000$ \\
\hline 1937 & $1,468,200,000$ & $3,200,500,000$ & $744,900,000$ & $3,945,400,000$ & $5,413,600,000$ \\
\hline $1938^{2}$ & $891,800,000$ & $2,804,700,000$ & $657,500,000$ & $3,462,200,000$ & $4,354,000,000$ \\
\hline
\end{tabular}

\section{Cement}

The cement industry depends for markets directly upon construction. Concrete highway paving and building, the two principal outlets, consume more than half of the output of portland cement manufactured in an average year. The remainder is consumed by other forms of construction such as sidewalks, bridges, dams, sewage and waterworks, railway maintenance, miscellaneous jobs on farms, and river and harbor improvements.

In Iowa, cement followed the general pattern of recovery from the depression, although recovery was somewhat slower than for some other mineral products. It continued a downward trend through 1933 but in that year suggestions of the recovery to follow were in evidence. The average price per barrel at the plant increased more than 48 per cent in 1933, 15.9 additional per cent in 1934 and since then has maintained a fairly steady level. The peak price of $\$ 1.58$ was reached in 1935. Shipments, after a decrease in 1933 caused in part by advanced price, increased in quantity and value each year except 1935. In 1934 and 1936 the quantity of Iowa cement shipped, represented increases of 21 per cent and 38 per cent respectively over shipments of the previous year. Production, after 1933, increased each year with the largest quantity advances in 1935 (11 per cent), 1936 (16 per cent) and 1937 (15 per cent).

The condition of the industry in Iowa for the period 1935-1938 was better than for the country as a whole. A comparison of the statistics for Iowa (Table III) and for the United States (Table IV) shows that 
TABLE III

Production of Cement in Iowa from 1932 to 1938

\begin{tabular}{|c|c|c|c|c|c|c|c|c|c|c|}
\hline \multirow[b]{2}{*}{ Year } & \multicolumn{2}{|c|}{ Production } & \multicolumn{4}{|c|}{ Shipments } & \multicolumn{2}{|c|}{ Stock at Mills Dec. 31} & \multirow[b]{2}{*}{$\begin{array}{c}\text { Annual ca- } \\
\text { pacity }\end{array}$} & \multirow[b]{2}{*}{$\begin{array}{l}\text { Plants } \\
\text { active }\end{array}$} \\
\hline & Barrels & $\begin{array}{l}\text { Per cent } \\
\text { change } \\
\text { over previ- } \\
\text { ous year }\end{array}$ & Barrels & Value & $\begin{array}{c}\text { Ayerage } \\
\text { price per } \\
\text { barrel }\end{array}$ & $\begin{array}{l}\text { Per cent } \\
\text { change in } \\
\text { quantity } \\
\text { over previ- } \\
\text { ous year } \\
\end{array}$ & Barrels & $\begin{array}{c}\text { Per cent } \\
\text { change } \\
\text { over previ- } \\
\text { ous year }\end{array}$ & & \\
\hline $\begin{array}{l}1932 \\
1933 \\
1934 \\
1935 \\
1936 \\
1937 \\
1938\end{array}$ & $\begin{array}{l}4,270,739 \\
3,044,008 \\
3,180,546 \\
3,519,558 \\
4,099,121 \\
4,706,094 \\
4,726,517\end{array}$ & $\begin{array}{l}-26 \\
-29 \\
+4 \\
+11 \\
+16 \\
+15 \\
+0.4\end{array}$ & $\begin{array}{l}4,373,642 \\
2,770,656 \\
3,340,049 \\
3,203,301 \\
4,407,624 \\
4,598,453 \\
4,759,390\end{array}$ & $\begin{array}{r}\$ 3,907,427 \\
3,651,921 \\
5,094,922 \\
5,072,098 \\
6,908,225 \\
7,046,021 \\
7,327,048\end{array}$ & $\begin{array}{r}\$ 0.89 \\
1.32 \\
1.53 \\
1.58 \\
1.57 \\
1.53 \\
1.54\end{array}$ & $\begin{array}{l}-23 \\
-37 \\
+21 \\
-4 \\
+38 \\
+4 \\
+3\end{array}$ & $\begin{array}{l}1,311,583^{\mathrm{a}} \\
1,605,116^{\mathrm{a}} \\
1,445,613^{\mathrm{a}} \\
1,761,870 \\
1,462,146 \\
1,569,787 \\
1,536,914\end{array}$ & $\begin{array}{l}-7 \\
+22 \\
-10 \\
+22 \\
+17 \\
+7 \\
-2\end{array}$ & $\begin{array}{l}10,293,900 \\
10,006,710 \\
10,066,710\end{array}$ & $\begin{array}{l}5 \\
5 \\
5 \\
5 \\
5 \\
5 \\
5\end{array}$ \\
\hline
\end{tabular}

TABLE IV

Production of Cement in the United States from 1932 to 1938

\begin{tabular}{|c|c|c|c|c|c|c|c|c|}
\hline \multirow[b]{2}{*}{ Year } & \multicolumn{2}{|c|}{ Production } & \multicolumn{4}{|c|}{ Shipments } & \multicolumn{2}{|c|}{ Stock at Mills, December 31} \\
\hline & Barrels & $\begin{array}{c}\text { Per cent change } \\
\text { over previous } \\
\text { year }\end{array}$ & Barrels & Value & $\begin{array}{l}\text { Average factory } \\
\text { value per barrel }\end{array}$ & $\mid \begin{array}{l}\text { Per cent change } \\
\text { in quantity over } \\
\text { previous year }\end{array}$ & Barrels & $\begin{array}{l}\text { Per cent change } \\
\text { over previous } \\
\text { year }\end{array}$ \\
\hline $\begin{array}{l}1932 \\
1933 \\
1934 \\
1935 \\
1936 \\
1937 \\
1938\end{array}$ & $\begin{array}{r}76,740,945 \\
63,473,189 \\
77,747,765 \\
76,741,570 \\
112,649,782 \\
116,174,708 \\
105,357,000\end{array}$ & $\begin{array}{l}-39 \\
-17 \\
+22 \\
-1 \\
+47 \\
+3 \\
-9\end{array}$ & $\begin{array}{r}80,843,187 \\
64,282,756 \\
75,901,279 \\
75,232,917 \\
112,849,979 \\
113,804,782 \\
106,324,127\end{array}$ & $\begin{array}{r}\$ 82,021,723 \\
85,583,916 \\
116,921,084 \\
113,372,182 \\
170,415,302 \\
168,835,208 \\
153,977,226\end{array}$ & \begin{tabular}{|c|}
$\$ 1.01$ \\
1.33 \\
1.54 \\
1.51 \\
1.51 \\
1.48 \\
1.45
\end{tabular} & $\begin{array}{l}-36 \\
-20 \\
+18 \\
-1 \\
+50 \\
+1 \\
-7\end{array}$ & $\begin{array}{l}20,351,058^{\mathrm{a}} \\
19,605,323^{\mathrm{a}} \\
21,440,594 \mathrm{a} \\
22,949,247^{\mathrm{a}} \\
22,568,685 \\
24,938,612 \\
23,946,118\end{array}$ & $\begin{array}{l}-12 \\
-4 \\
+9 \\
+7 \\
-2 \\
+11 \\
-4\end{array}$ \\
\hline
\end{tabular}

a Revised. 
Iowa productions, shipments and prices were below average for 1933 and 1934. In the years following, however, Iowa cement had a more steady advancement than cement in the United States in general. It is notable that the price was from 5 to 9 cents per barrel above the United States figures and that there was no reduction in the quantity or value of Iowa shipments, production or price in 1938 when all of these decreased for the country as a whole.

Production of cement in the United States was highly variable during the period of this report. It was far below the general level of business in 1932 and 1933, and in 1933 shipments reached the lowest level in twenty-five years, although in that year there was a marked general increase in price.

The decline in United States cement production in 1933 was caused by the sharp drop in concrete paving contract awards and the continued slump in building construction. The increases in construction contract awards, due largely to Public Works Administration activity, arrived too late in the year to prevent the decline. They are reflected, however, in the advance in shipments in 1934 in spite of the fact that residential building remained at a low level. Production and shipments fell off in 1935 and in that year two new plants were put into operation, the first new plants since 1929.

In 1936 there were increases of 47 per cent in quantity and 50 per cent in value. These advances were brought about by extensive highway building, large Federal Public Works projects and renewed activity in private construction. There was a slight increase in production and quantity shipped in 1937, but the value of shipments declined because of the 2-per cent drop in price. The average price per barrel increased almost 32 per cent in 1933, 16 per cent in 1934 and decreased 2 per cent each year in 1935, 1937 and 1938. In the last named year, production and shipments also fell off although during the last quarter of 1938 figures were above those for the same period in 1937 and the outlook appeared bright.

\section{Manufacturers of Portland Cement in Iowa}

\section{Cerro Gordo County}

Lehigh Portland Cement Co., B. L. Swett, Vice President, Young Building, Allentown, Pa. Plant at Mason City, Iowa.

Northwestern States Portland Cement Co., Peter Andersen, Secre- 
tary, First National Bank Building, Mason City, Iowa. Plant at Mason City, Iowa.

Pocahontas County

Northwestern States Portland Cement Co., First National Bank

Building, Mason City, Iowa. Plant at Gilmore City, Iowa.

Polk County

Hawkeye Portland Cement Co., B. E. Manley, General Manager, Hubbell Building, Des Moines, Iowa. Plant at Des Moines, Iowa.

Pennsylvania-Dixie Cement Corp., R. A. Bechtold, Superintendent, 208 Old Colony Building, N. E. Corner 10th \& Grand Avenue, Des Moines, Iowa. Plant at West Des Moines, Iowa.

Scott County

Dewey Portland Cement Co., F. E. Tyler, President, 101 West 11th Street, Kansas City, Missouri. Plant at Davenport, Iowa.

\section{Clay}

Clay, which suffered more serious declines than any other Iowa mineral product during the early part of the depression, rallied more strongly than any other product except limestone during the period 1933-1938. Total value increased over the previous year in 1933 and in each of the succeeding years except 1938. The greatest advances as shown by Table $\mathrm{V}$ were attained in 1934 and 1935 when they amounted to 49.8 per cent and 48.4 per cent respectively, and were never less than the 13.8 per cent gain in 1933, while the decline in 1938 amounted to only 11.7 per cent. The value in 1937 was more than four times that in 1932 .

Statistics for most mineral products are collected by the Bureau of Mines cooperating with the state geological surveys. These agencies obtain data concerning the amount of clay sold either raw or prepared, but not made into wares. For clay wares the canyasses are made without the cooperation of the state surveys by the Bureau of Census, which reports clay products by class, quantity, and value. It is not known if the same producers are reached by both canvasses.

Sales of the various raw and prepared clays in Iowa followed a strong upward trend in total quantity and total value, but individually were extremely erratic during the period of this report. For example, fire clay in 1933 increased more than 730 per cent in quantity and more than 850 per cent in value, in 1934 decreased 82 per cent in 
TABLE V

Value of Clay Products and Raw Clay in Iowa from 1932 to 1938

\begin{tabular}{|c|c|c|c|c|c|c|c|}
\hline & 1932 & 1933 & 1934 & 1935 & 1936 & 1937 & 1938 \\
\hline $\begin{array}{l}\text { Clay products } \\
\text { Clay, raw }\end{array}$ & $\begin{array}{r}\$ 796,445 \\
9,354\end{array}$ & $\begin{array}{r}\$ 842,726 \\
74,822\end{array}$ & $\begin{array}{r}\$ 1,352,227 \\
22,242\end{array}$ & $\begin{array}{r}\$ 2,006,021 \\
33,547\end{array}$ & $\begin{array}{r}\$ 2,728,810 \\
46,023\end{array}$ & $\begin{array}{r}\$ 3,250,677 \\
50,871\end{array}$ & $\begin{array}{r}\$ 2,868,233 \\
45,759\end{array}$ \\
\hline Total & 805,799 & 917,548 & $1,374,469$ & $2,039,568$ & $2,774,833$ & $3,301,548$ & $2,913,992$ \\
\hline
\end{tabular}

TABLE VI

Sales of Clay in Iowa from 1932 to 1938

\begin{tabular}{|c|c|c|c|c|c|c|c|}
\hline & 1932 & 1933 & 1934 & 1935 & 1936 & 1937 & 1938 \\
\hline $\begin{array}{l}\text { Fire Clay } \\
\text { Short tons } \\
\text { Value } \\
\text { Active producers }\end{array}$ & $\begin{array}{r}858 \\
\$ 7,255 \\
5\end{array}$ & $\begin{array}{r}7,158 \\
\$ 69,169 \\
6\end{array}$ & $\begin{array}{r}1,255 \\
\$ 11,651 \\
6\end{array}$ & $\begin{array}{r}2,143 \\
\$ 20,726 \\
6\end{array}$ & $\begin{array}{r}1,094 \\
\$ 12,635 \\
3\end{array}$ & $\begin{array}{l}\text { (a) } \\
\text { (a) } \\
2\end{array}$ & $\begin{array}{r}773 \\
\$ 9,034 \\
3\end{array}$ \\
\hline $\begin{array}{l}\text { Miscellaneous Clay } \\
\text { Short tons } \\
\text { Value } \\
\text { Active producers }\end{array}$ & $\begin{array}{r}2,575 \\
\$ 2,099 \\
2\end{array}$ & $\begin{array}{r}2,221 \\
\$ 5,653 \\
5\end{array}$ & $\begin{array}{r}1,017 \\
\$ 10,591 \\
5\end{array}$ & $\begin{array}{r}1,132 \\
\$ 12,821 \\
3\end{array}$ & $\begin{array}{c}3,317 \mathrm{~b} \\
\$ 33,388 \\
7\end{array}$ & $\begin{array}{c}(a) \\
+(a) \\
7\end{array}$ & $\begin{array}{r}6,055 \\
\$ 36,725 \\
8\end{array}$ \\
\hline $\begin{array}{l}\text { Short tons } \\
\text { Value } \\
\text { Active producers }\end{array}$ & $\begin{array}{r}3,433 \\
\$ 9,354 \\
6\end{array}$ & $\begin{array}{r}9,379^{\circ} \\
\$ 74,822 \\
10\end{array}$ & $\begin{array}{r}2,272 \\
\$ 22,242 \\
10\end{array}$ & $\begin{array}{r}3,275 \\
\$ 33,547 \\
9\end{array}$ & $\begin{array}{r}4,411 \\
\$ 46,023 \\
10\end{array}$ & $\begin{array}{r}4,600 \\
\$ 50,871 \\
8\end{array}$ & $\begin{array}{r}6,828 \\
\$ 45,759 \\
10\end{array}$ \\
\hline
\end{tabular}

Witheld to avoid disclosing, exactly or approximately, data reported by individual establishments.

b Includes ground clay, modeling clay and shale. 
quantity and 83 per cent in value, in 1935 increased 71 per cent in quantity and 78 per cent in value and again decreased in 1936 and 1938. Clays listed as "miscellaneous" also varied, but not as greatly as did the fire clay. The apparent discrepancy between the annual quantities and values of miscellaneous clays is due to difference in price of the various clays included under this general heading. Table VI presents the salient features of this portion of the clay industry.

Clay products after disastrous reversals, particularly in 1932, began a recovery in 1933 which resulted in a material increase in total value each year until 1938. The decline in 1938 was not serious and the total value for that year was higher than for 1936.

The production of clay products by classes in 1935 and 1936 is shown by Table VII. Hollow partition building tile leads all other

TABLE VII

Production of Clay Products in Iowa by Class, Quantity and Value, 1935-1936

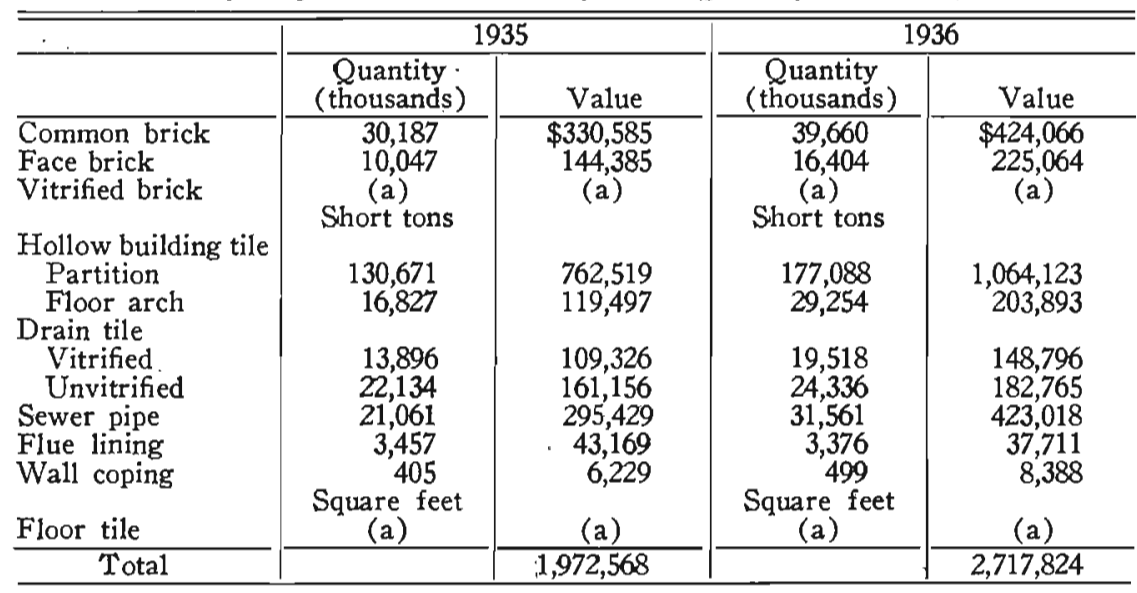

a Concealed to avoid revealing confidential information.

classes by a considerable margin and was followed in value by common brick, sewer pipe, face brick, and unvitrified drain tile in the order named. Detailed figures for clay production during 1933-1934 and 1937-1938 were not available when this report went to press.

The domestic potteries industries in the United States were relatively inactive in 1933. There was a sharp decline in sales of clay for stoneware, but recoveries in paper making and refractories manufacture reflected in clay sales for those uses. Fire clay and bentonite increased about 50 per cent. In 1934 shipments in virtually all kinds of clay began or continued to recover, and except for stoneware, con- 
tinued this trend in 1935. Advances were even more marked for 1936 when production and sales of domestic kaolin broke all previous records. In 1937 china clay production and sales and ball clay shipments broke all previous records and fire clay sales. were greater than for any earlier year, except 1929. Heavy clay products likewise improved further in 1937, but the recession made itself felt during the last quarter of 1937 and in early 1938 there were drastic reversals of the progress which the clay industry had made during the previous few years, although heavy clay products fell off only slightly. Buildingcontract awards increased substantially during the latter part of 1938 and there appeared to be every indication that clay production would again advance in 1939 .

\section{Clay Operators in Iowa}

\section{Cerro Gordo County}

Lehigh Portland Cement Co., Young Building, Allentown, Pa. Mine at Mason City, Iowa.

Mason City Brick and Tile Co., 19 West State Street, Mason City, Iowa.

- Dallas County

Adel Clay Products Co., Adel, Iowa. Mine at Redfield, Iowa. Floyd County

Rockford Brick \& Tile Co., Rockford, Iowa.

Keokuk County

What Cheer Clay Products Co., What Cheer, Iowa.

Mahaska County

Maria Hansel, Oskaloosa, Iowa.

Polk County

Goodwin Tile \& Brick Co., Box 283, S. E. 18th \& Hartford Avenue, Des Moines, Iowa.

Wapello County

Morey Clay Products Co., Ottumwa, Iowa.

Webster County

Johnston Clay Works, Inc., 214 First National Bank Building, Fort Dodge, Iowa. Clay works near Fort Dodge, Iowa.

Kalo Brick \& Tile Co., 603 Snell Building, Fort Dodge, Iowa. Mine at Coalville, Iowa.

George F. Drain, Lehigh, Iowa. 
TABLE VIII

Summary of the Bituminous-coal Industry in Iozea from 1932 to 1938

\begin{tabular}{|c|c|c|c|c|c|c|c|c|}
\hline \multirow[b]{2}{*}{ r } & \multirow[b]{2}{*}{$\begin{array}{l}\text { Production } \\
\text { in net tons }\end{array}$} & \multicolumn{2}{|c|}{ Value } & \multicolumn{3}{|c|}{ Number of Employees } & \multirow{2}{*}{$\begin{array}{l}\text { Average num- } \\
\text { ber of days } \\
\text { mine operated }\end{array}$} & \multirow{2}{*}{$\begin{array}{c}\text { Average } \\
\text { tons per man } \\
\text { per day }\end{array}$} \\
\hline & & Total & $\begin{array}{l}\text { Average } \\
\text { per ton }\end{array}$ & Underground & Surface & Total & & \\
\hline $\begin{array}{l}1932 \\
1933 \\
1934 \\
1935 \\
1936 \\
1937 \\
1938\end{array}$ & $\begin{array}{l}3,862,435 \\
3,194,983 \\
3,366,992 \\
3,650,163 \\
3,960,700^{\mathrm{c}} \\
3,637,054^{\mathrm{a}} \\
3,250,000^{\mathrm{ce}}\end{array}$ & $\begin{array}{c}\$ 9,254,000 \\
7,217,000 \\
7,862,000 \\
9,002,000 \\
-9,940,000^{\text {cd }} \\
9,529,000^{\mathrm{cd}} \\
8,340,000^{\mathrm{de}}\end{array}$ & $\begin{array}{l}\$ 2.40 \\
2.26 \\
2.34 \\
2.47 \\
2.51 \mathrm{~cd} \\
2.62^{\mathrm{cd}} \\
2.56^{\mathrm{de}}\end{array}$ & $\begin{array}{l}7,183 \\
6,591 \\
6,687 \\
6,998 \\
7,575^{\mathrm{c}} \\
\text { (f) } \\
\text { (f) }\end{array}$ & $\begin{array}{c}903 \\
1,104 \\
1,034 \\
1,040 \\
1,166^{c} \\
\text { (f) } \\
\text { (f) }\end{array}$ & $\begin{array}{l}8,086 \\
7,695 \\
7,721 \\
8,038 \\
8,741 \mathrm{c} \\
8,720^{\mathrm{bc}} \\
\text { (f) }\end{array}$ & \begin{tabular}{|c|}
151 \\
138 \\
156 \\
162 \\
$163^{\circ}$ \\
$146^{\circ}$ \\
(f)
\end{tabular} & $\begin{array}{c}3.17 \\
3.01 \\
2.80 \\
2.80 \\
2.78^{\mathrm{c}} \\
2.87^{\mathrm{c}} \\
\text { (f) }\end{array}$ \\
\hline
\end{tabular}

a Total production including inventory change and coal unaccounted for.

b Average number of employces.

d Estimated from various sources, includes selling expenses, and is not comparable with values of earlier years.

Not available. 
Lehigh Sewer Pipe \& Tile Co., Fort Dodge, Iowa. Mine at Lehigh, Iowa.

Vincent Clay Products Co., 617 State Bank Building, Fort Dodge, Iowa.

\section{Coal}

Coal is the most valuable mineral resource of Iowa on which statistics are available. Normally coal production represents over 40 per cent of the total annual mineral output of the State and because of the markets which it supplies, it is usually one of the most stable of mineral products. For the period of this report it did not react as favorably in general as did some of the other minerals in Iowa. It reached the bottom of the depression low in 1933, one year later than coal over the country as a whole, recovered in 1934, 1935 and 1936, and fell off in 1937 a year before the recession was felt in United States production. However, during the period of this report the price per ton was from $\$ 1.01$ to $\$ 0.69$ above the United States average.

Statistics on the general condition of the industry in Iowa may be found in Table VIII and for the United States in Table IX. The data

TABLE IX

Summary of Bituminous-coal Industry in the United States from 1932 to 1938

\begin{tabular}{|c|c|c|c|c|c|c|}
\hline & \multirow[b]{2}{*}{ Production } & \multirow{2}{*}{$\begin{array}{l}\text { Value at } \\
\text { mines }\end{array}$} & \multirow{2}{*}{$\begin{array}{l}\text { Average } \\
\text { value } \\
\text { per ton }\end{array}$} & \multicolumn{2}{|c|}{ Stocks ${ }^{a}$} & \multirow{2}{*}{$\begin{array}{l}\text { Consumption } \\
\text { (calculated) }\end{array}$} \\
\hline & & & & January 1 & December 31 & \\
\hline 1932 & $309,709,872$ & $\$ 406,677,000$ & $\$ 1.31$ & $35,500,000$ & $29,666,000$ & $306,917,000$ \\
\hline 1933 & $333,630,533$ & $445,788,000$ & 1.34 & $29,666,000$ & $32,840,000$ & $321,748,000$ \\
\hline 1934 & $359,368,022$ & $628,112,000$ & $1.75^{\mathrm{b}}$ & $32,840,000$ & $34,476,000$ & $347,043,000$ \\
\hline 1935 & $372,373,122$ & $658,063,000$ & 1.77 & $34,476,000$ & $37,017,000$ & $360,291,563$ \\
\hline 1936 & $439,087,903$ & $772,794,709 c$ & 1.76 & $37,017,000$ & 42926,000 & $422,795,741$ \\
\hline 1937 & $445,531,449$ & $868,786,325 \mathrm{c}$ & $1.95^{\mathrm{d}}$ & $42,926,000$ & $47,074,000$ & $428,496,767^{e}$ \\
\hline 1938 & $344,630,000^{t}$ & & & $47,074,000$ & $40,720,000^{t}$ & $340,735,036^{1}$, e \\
\hline
\end{tabular}

a Commercial consumers and retail yards.

b F. O. B. mine.

c Arrived at by multiplying figure for "production" by figure for "average value per ton."

d Average gross realization including selling price. Not comparable with years prior to 1937.

- Production plus imports minus exports plus or minus' changes in consumers stock.

P Preliminary.

used in coal reports for all years pertain only to commercial mines with an annual output of 1,000 tons or more. No figures comparable to earlier years are available after 1935 for total value and price per ton except as noted.

In 1933 the total quantity of Iowa coal declined 667,452 net tons or about 17 per cent from the 1932 figure, the price fell 14 cents per ton to a depression low of $\$ 2.26$, and the total value decreased over 
two millions of dollars while the number of men employed decreased 5 per cent.

Recovery began in 1934 when production, total value, price, number of men employed, and average number of days mines operated increased over the preceding year. In 1935. there were further increases in all phases of the industry which carried over through 1936 when production, value and the number of men employed were all greater than for 1932 and the ensuing years. The recession made itself felt in 1937 when there was a decrease in production, operating time, and men employed. Production and other detailed statistics by counties for 1938 were not available when this report went to press.

More detailed information on production by counties may be found in Tables X to XIV. By way of general summary of these tables, the leading counties in production and value are listed below in the order of rank.

\begin{tabular}{|c|c|}
\hline & \\
\hline 33 Total quantity: & Polk, I,ucas, Boone, Marion, \\
\hline Total value: & Polk, Boone, Irucas, Dallas, \\
\hline 1934 Total quantity: & Appanoose, Boone, \\
\hline Total value: & $\begin{array}{l}\text { Dallas, Monree } \\
\text { Polk, Lucas, Boone, Appanoose, } \\
\text { Dallas, Monroe }\end{array}$ \\
\hline 1935 Total quantity: & $\begin{array}{l}\text { Appanoose, Polk, Lucas, Boone, } \\
\text { Dallas, Marion }\end{array}$ \\
\hline Total value: & $\begin{array}{l}\text { Appanoose, Polk, Boone, Lucas, } \\
\text { Dallas, Marion }\end{array}$ \\
\hline 1936 Total quantity: & $\begin{array}{l}\text { Appanoose, Lucas, Polk, Boone, } \\
\text { Dallas, Marion }\end{array}$ \\
\hline & $\begin{array}{l}\text { Appanoose, Marion, Polk, Lucas } \\
\text { Dallas, Boone }\end{array}$ \\
\hline
\end{tabular}

The bituminous-coal industry in the United States, a summary of which appears in Table IX, started an upswing in 1933 which carried through 1937. Total production increased about 22 millions of tons in 1933, over 26 millions in 1934, about 13 millions in 1935 and almost 67 millions in 1936, but dropped almost 101 millions of tons in 1938 according to the preliminary data for that year. The average value per ton increased from $\$ 1.31$ in 1932 to $\$ 1.77$ in 1935 , which represents an advance of 35 per cent. The greatest annual increase in price came in 1934 when the average value reached $\$ 1.75$ per ton as compared to $\$ 1.34$ for 1933 . For a more complete review of coal production by states for the period 1933-1937 see Tables XV-XIX.

The advance in price and improvement in the employment situation was at least partly due to National Recovery Administration activities 
TABLE X

Production, Value, Men Employed, Days Operated, and Output PerMan Per Day at Bituminous-coal Mines in Iowa in 1933

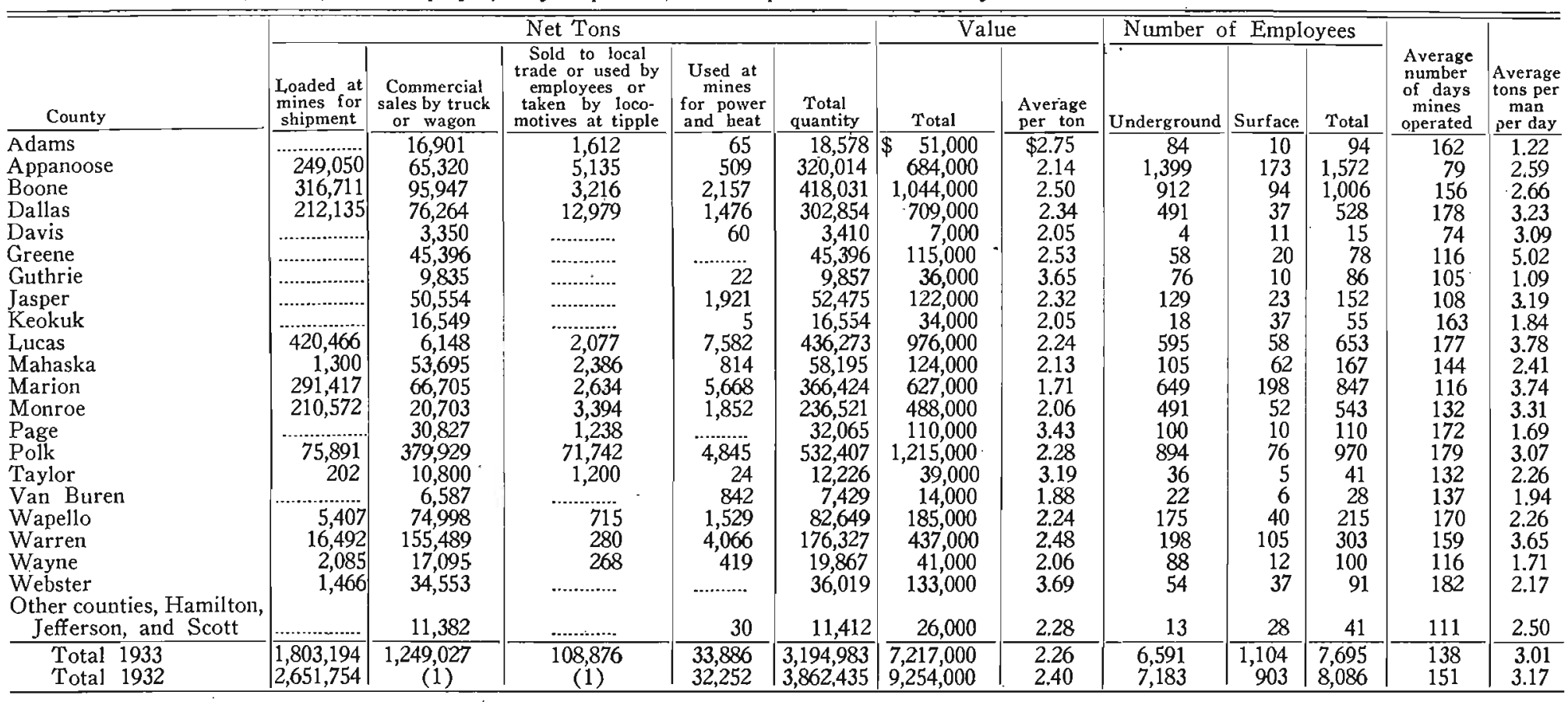

(1) In 1932 there were 248,225 tons reported by the operator as "trucked 10 miles or more from mine" (including 20,848 tons, a part of which went less than 10 miles,

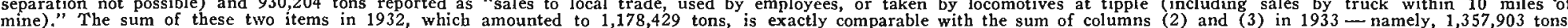


TABLE XI

Production, Value, Men Employed, Days Operated and Output Per Man Per Day at Bituminous-coal Mines in Iowa in 1934

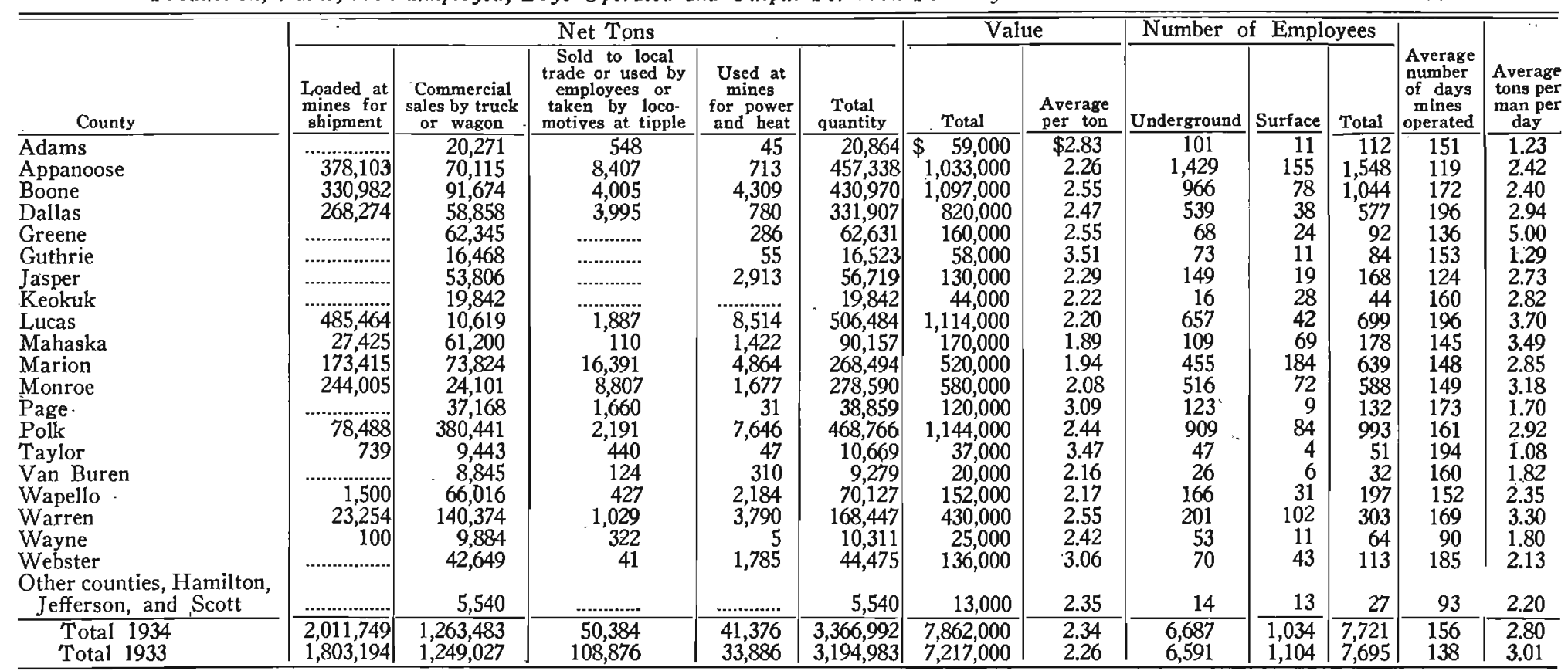


TABLE XII

Production, Value, Men Employed, Days Operated, and Output Per Man Per Day at Bituminous-coal Mines in Ioze in 1935

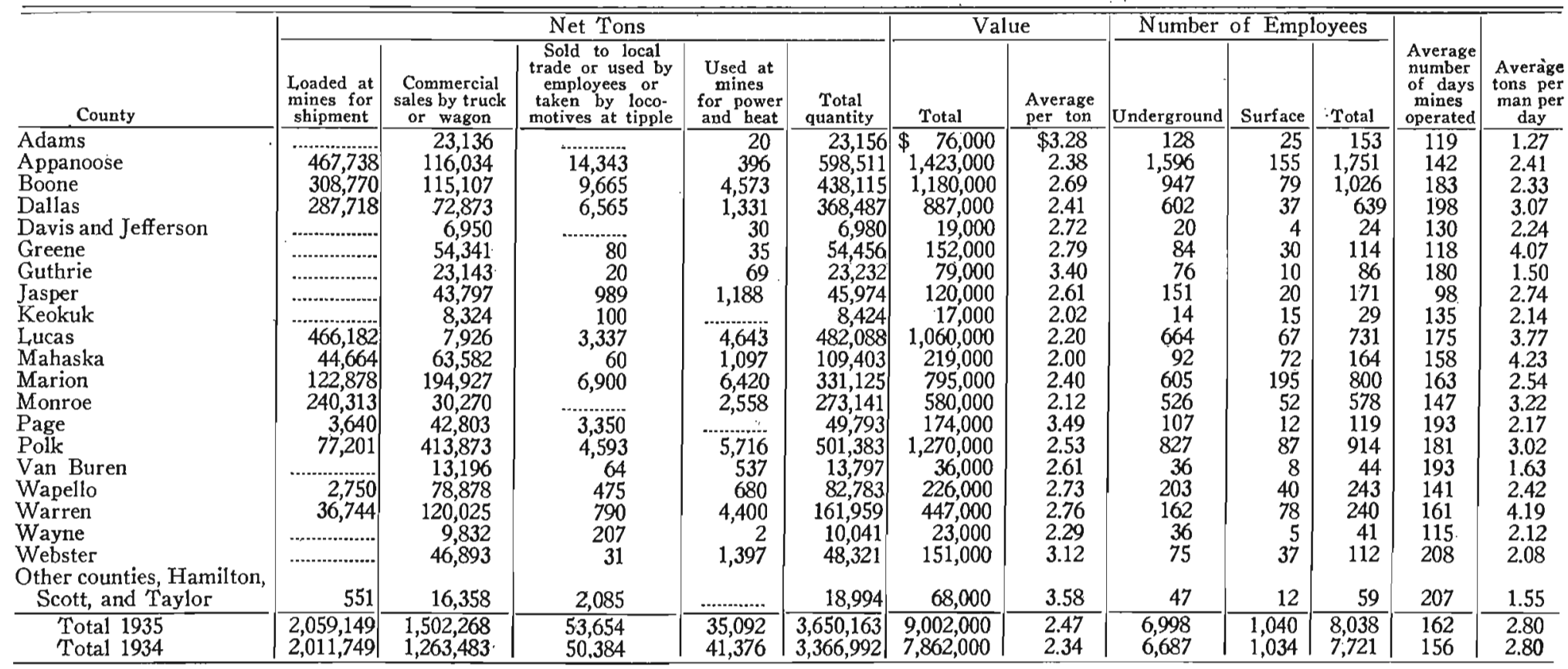


TABLE XIII

Production, Men Employed, Days Operated and Output Per Man Per Day at Bituminous-coal Mines in Iowa in 1936

\begin{tabular}{|c|c|c|c|c|c|c|c|c|c|c|}
\hline \multirow[b]{2}{*}{ County } & \multicolumn{5}{|c|}{ Net Tons } & \multicolumn{3}{|c|}{ Number of Employees } & \multirow[b]{2}{*}{$\begin{array}{c}\text { Average } \\
\text { number } \\
\text { of days } \\
\text { mines } \\
\text { operated }\end{array}$} & \multirow[b]{2}{*}{$\begin{array}{c}\text { Average } \\
\text { tons per } \\
\text { man per } \\
\text { day }\end{array}$} \\
\hline & $\begin{array}{l}\text { Loaded at } \\
\text { mines for } \\
\text { shipment }\end{array}$ & $\begin{array}{l}\text { Commercial } \\
\text { sales by truck } \\
\text { or wagon }\end{array}$ & $\begin{array}{l}\text { Sold to local } \\
\text { trade or used by } \\
\text { employees or } \\
\text { taken by loco- } \\
\text { motives at tipple }\end{array}$ & $\begin{array}{l}\text { Used at } \\
\text { mines } \\
\text { for power } \\
\text { and heat }\end{array}$ & $\begin{array}{c}\text { Total } \\
\text { quantity }\end{array}$ & Underground & Surface & Total & & \\
\hline Adams & & 30,299 & & 38 & 30,337 & 181 & 24 & 205 & 128 & 1.16 \\
\hline Appanoose & 483,876 & 158,059 & 14,689 & 3,312 & 659,936 & 1,762 & 196 & 1,958 & 138 & 2.44 \\
\hline Boone & 297,451 & 142,030 & 1,646 & 3,822 & 444,949 & 960 & 84 & 1,044 & 182 & 2.34 \\
\hline Dallas & 298,356 & 93,398 & 5,756 & 1,407 & 398,917 & 632 & 47 & 679 & 184 & 3.19 \\
\hline Davis and Jefferson & ………....... & 21,525 & 27 & 10 & 21,562 & 26 & 17 & 43 & 168 & 2.98 \\
\hline Greene & ................. & $.54,996$ & 62 & 220 & 55,278 & 71 & 26 & 97 & 137 & 4.17 \\
\hline Guthrie & ...................... & 29,887 & & 39 & 29,926 & 118 & 10 & 128 & 155 & 1.51 \\
\hline Jasper & ……............. & 57,789 & 200 & 2,547 & 60,536 & 175 & 26 & 201 & 112 & 2.70 \\
\hline Keokuk & & 8,244 & & 25 & 8,269 & 10 & 17 & 27 & 100 & 3.07 \\
\hline Lucas & 531,748 & 20,190 & 4,248 & 4,541 & 560,727 & 676 & 66 & 742 & 189 & 4.00 \\
\hline Mahaska & 60,163 & 91,209 & 76 & 1,026 & 152,474 & 167 & 122 & 289 & 138 & 3.83 \\
\hline Marion & 103,533 & 241,919 & 15,364 & 5,897 & 366,713 & 583 & 189 & 772 & 174 & 2.72 \\
\hline Monroe & 177,529 & $-62,577$ & 3,369 & 2,482 & 245,957 & 424 & 47 & 471 & 168 & 3.11 \\
\hline Page & & 40,990 & 5,112 & 37 & 46,139 & 108 & 9 & 117 & 193 & 2.04 \\
\hline Polk & 93,068 & 428,035 & 4,174 & 5,792 & 531,069 & 937 & 106 & 1,043 & 178 & 2.86 \\
\hline Taylor & 1,910 & 13,124 & 595 & 6 & 15,635 & 56 & 6 & 62 & 191 & 1.32 \\
\hline Van Buren & & 13,354 & 54 & 612 & 14,020 & 44 & 11 & 55 & 162 & 1.57 \\
\hline Wa pello & 2,400 & 97,445 & 512 & 2,239 & 102,596 & 267 & 48 & 315 & 146 & 2.24 \\
\hline Warren & 21,672 & 109,475 & 448 & 4,332 & 135,927 & 193 & 69 & 262 & 154 & 3.37 \\
\hline Wayne & . & 28,884 & 334 & 425 & 29,643 & 113 & 16 & 129 & 148 & 1.55 \\
\hline Webster & & 49,477 & 43 & 570 & 50,090 & 72 & 30 & 102 & 212 & 2.32 \\
\hline Total 1936 & $2,071,706$ & $1,792,906$ & 56,709 & 39,379 & $3,960,700$ & 7,575 & $1, \overline{166}$ & 8,741 & 163 & 2.78 \\
\hline Total 1935 & $2,059,149$ & $1,502,268$ & 53,654 & 35,092 & $3,650,163$ & 6,998 & 1,040 & 8,038 & 162 & 2.80 \\
\hline
\end{tabular}


TABLE XIV

Production, Men Employed, Days Operated, and Output Per Man Per Day at Bituminous-coal Mines in Iowa in 1937

\begin{tabular}{|c|c|c|c|c|c|c|c|c|}
\hline \multirow[b]{2}{*}{ County } & \multicolumn{5}{|c|}{ Net Tons } & \multirow[b]{2}{*}{$\begin{array}{l}\text { Average } \\
\text { number of } \\
\text { employees }\end{array}$} & \multirow[b]{2}{*}{$\begin{array}{c}\text { Average } \\
\text { number of } \\
\text { days mines } \\
\text { operated }\end{array}$} & \multirow[b]{2}{*}{$\begin{array}{l}\text { Average } \\
\text { tons per mas } \\
\text { per day }\end{array}$} \\
\hline & $\begin{array}{l}\text { Loaded at } \\
\text { mines for } \\
\text { shipment }\end{array}$ & $\begin{array}{l}\text { Truck deliv- } \\
\text { eries includ- } \\
\text { ing local sales }\end{array}$ & $\begin{array}{l}\text { Used by } \\
\text { mine } \\
\text { employees }^{2}\end{array}$ & Mine fuel & $\begin{array}{l}\text { Total produc- } \\
\text { tion including in- } \\
\text { ventory change } \\
\text { and coal unac- } \\
\text { counted for } 1 \\
\end{array}$ & & & \\
\hline Adams & & 28,250 & & 20 & 28,270 & 180 & 151 & 1.04 \\
\hline Appanoose & 442,498 & 156,388 & 3,871 & 175 & 602,846 & 1,925 & 131 & 2.39 \\
\hline Boone & 236,592 & 97,507 & 7,918 & 1,913 & 343,930 & 987 & 142 & 2.45 \\
\hline Dallas & 286,228 & 96,098 & 7,394 & 1,720 & 393,047 & 703 & 182 & 3.08 \\
\hline Greene & ………............ & 40,392 & ................. & 230 & 40,622 & 84 & 102 & 4.30 \\
\hline Guthrie & .................... & 22,552 & & 18 & 22,570 & 101 & 156 & 1.43 \\
\hline Jasper & ....................... & 42,271 & 100 & 1,698 & 44,069 & 158 & 91 & 3.06 \\
\hline Keokuk & & 10,181 & & 25 & 10,206 & 26 & 94 & 4.18 \\
\hline Lucas & 404,974 & 14,602 & 7,576 & 4,766 & 431,918 & 825 & 133 & 3.94 \\
\hline Mahaska & 91,009 & 136,007 & 1,435 & 1,731 & 230,297 & 282 & 174 & 4.71 \\
\hline Marion & 72,743 & 392,961 & 2,892 & 2,113 & 470,955 & 916 & 156 & 3.29 \\
\hline Monroe & 108,069 & 72,588 & 7,172 & 1,681 & 189,352 & 387 & 156 & 3.13 \\
\hline Page & & 39,563 & & 25 & 39,588 & 143 & 162 & $1: 71$ \\
\hline Polk & 65,162 & 360,988 & 4,175 & 5,232 & 435,557 & 1,039 & 153 & 2.74 \\
\hline Van Buren & 64 & 18,121 & & 40 & 18,225 & 52 & 169 & 2.08 \\
\hline Wapello & 12,275 & 120,397 & 100 & 1,627 & 134,414 & 353 & 140 & 2.73 \\
\hline Warren & 11,189 & 91,473 & 1,711 & 2,668 & 107,019 & 270 & 116 & 3.41 \\
\hline Wayne & & 22,530 & 400 & 30 & 22,960 & 104 & 150 & 1.47 \\
\hline Webster & ……............... & 36,468 & ............ & 190 & 36,658 & 91 & 164 & 2.45 \\
\hline Jefferson and Taylor) & 370 & 33,668 & 493 & 20 & 34,551 & 94 & 163 & 2.25 \\
\hline Total Iowa, 1937 & $1,731,17 \overline{3}$ & $1,833,005$ & 45,237 & $25,922^{(2)}$ & $3,637,054$ & 8,720 & 146 & 2.87 \\
\hline Total Iowa, 1936 & $2,071,706$ & $1,792,906$ & 56.709 & 39,379 & $3,960,700$ & 8,741 & 163 & 2.78 \\
\hline
\end{tabular}

1 The total production differs from the sum of the items shown by the amount of the changes in inventory and of tonnage not accounted for in the distribution

2 Other sales to local trade or used by employees or taken by locomotives at tipple.
analysis. 
which began in 1933, carried through 1934, but on May 22, 1935 was invalidated in the courts. The price structure of coal suffered for a time, but was aided by the Guffey Act passed on August 20, 1935 and the subsequent appointment of the National Bituminous Coal Commission.

After passing the Bituminous Coal Act of 1937, Congress discontinued the appropriation for collection of bituminous coal statistics by the Bureau of Mines, since such work would thereafter center in the Bituminous Coal Commission.

The Commission changed the method of reporting "value per ton" by including selling expense, an item which was not included in the old method used by the Bureau of Mines in calculating this value. A comparison of the two series is possible for 1936. In that year the average value per ton, on the basis used by the Bureau of Mines was $\$ 1.76$, while the average gross realization, as collected by the Coal Commission, was $\$ 1.83$. Comparable figures are not available for later years.

In Tables XV to XIX presenting the summaries of coal production, value, men employed, days operated, and output per man per day by states, the average tons per man per day is based upon ( 1 ) the "reported" number of man-shifts where the operator keeps a record thereof; otherwise upon (2) the "calculated" number of man-shifts obtained by multiplying the average number of men employed underground and on the surface at each mine by the number of days worked by the mine and tipple, respectively. 
TABLE XV

Summary of Coal Produced, Value, Men Employed, Days Operated, and Output Per Man Per Day, by Slates, in 1933. (Exclusive of Product of Truck and Wagon Mines Producing Less Than One Thousand Tons)

\begin{tabular}{|c|c|c|c|c|c|c|}
\hline State & $\begin{array}{c}\text { Total } \\
\text { quantity }\end{array}$ & $\begin{array}{l}\text { Total } \\
\text { value }\end{array}$ & $\begin{array}{l}\text { Average } \\
\text { per ton }\end{array}$ & $\begin{array}{l}\text { Number of } \\
\text { employees }\end{array}$ & $\begin{array}{l}\text { Average number } \\
\text { of . days mines } \\
\text { operated }\end{array}$ & $\begin{array}{l}\text { Average tons } \\
\text { per man per day }\end{array}$ \\
\hline Alabama & $8,759,989$ & $\$ 13,758,000$ & $\$ 1.57$ & 18,237 & 148 & 3.26 \\
\hline Alaska & 96,467 & 481,000 & 4.99 & 100 & 199 & 4.86 \\
\hline Arizona & 10,345 & 52,000 & 5.03 & 23 & 268 & 1.68 \\
\hline Arkansas & 882,924 & $2,348,000$ & 2.66 & 3,671 & 94 & 2.57 \\
\hline California, Idaho and Oregon & 7,492 & 27,000 & 3.60 & 58 & 79 & 1.65 \\
\hline Colorado & $5,229,767$ & $11,350,000$ & 2.17 & 7,908 & 148 & 4.46 \\
\hline Georgia & 41,382 & 77,000 & 1.86 & 93 & 234 & 1.90 \\
\hline Illinois & $37,413,145$ & $54,578,000$ & 1.46 & 44,145 & 141 & 6.00 \\
\hline Indiana & $13,761,052$ & $17,567,000$ & 1.28 & 11,199 & 163 & 7.52 \\
\hline Iowa. & $3,194,983$ & $7,217,000$ & 2.26 & 7,695 & 138 & 3.01 \\
\hline Kansas & $2,217,622$ & $3,881,000$ & 1.75 & 3,809 & 140 & 4.15 \\
\hline Kentucky & $36,099,729$ & $40,748,000$ & 1.13 & 43,717 & 170 & 4.87 \\
\hline Maryland & $1,530,748$ & $2,134,000$ & 1.39 & 2,880 & 172 & 3.09 \\
\hline Michigan & 406,584 & $1,171,000$ & 2.88 & 1,186 & 130 & 2.63 \\
\hline Missouri & $3,432,212$ & $6,175,000$ & 1.80 & 5,690 & 150 & 4.02 \\
\hline Montana & $2,152,207$ & $3,309,000$ & 1.54 & 1,324 & 166 & 9.80 \\
\hline New Mexico & $1,226,236$ & $3,071,000$ & 2.50 & 2,340 & 168 & 3.12 \\
\hline North Carolina & 2,014 & 7,000 & 3.48 & 10 & 175 & 1.15 \\
\hline North Dakota & $1,782,272$ & $2,248,000$ & 1.26 & 1,301 & 173 & 7.93 \\
\hline Ohio & $19,588,763$ & $23,549,000$ & 1.20 & 25,442 & 169 & 4.55 \\
\hline Oklahoma & $1,238,244$ & $2,616,000$ & 2.11 & 2,974 & 128 & 3.26 \\
\hline Pennsylvania, bituminous & $79,295,944$ & $108,418,000$ & 1.37 & 115,453 & 162 & 4.24 \\
\hline South Dakota & 59,375 & 104,000 & 1.75 & 147 & 100 & 4.04 \\
\hline Tennessee & $3,774,761$ & $5,255,000$ & 1.39 & 7,051 & 161 & 3.33 \\
\hline Texas & 821,878 & $\begin{array}{r}833,000 \\
5100000\end{array}$ & 1.01 & 803 & 162 & 6.32 \\
\hline Utah & $2,674,986$ & $5,109,000$ & 1.91 & 2,906 & 176 & 5.23 \\
\hline Virginia & $8,178,642$ & $10,029,000$ & 1.23 & 9,761 & 184 & 4.55 \\
\hline Washington & $1,394,068$ & $3,916,000$ & 2.81 & 2,555 & 168 & 3.25 \\
\hline West Virginia & $94,343,535$ & $107,124,000$ & 1.14 & 92,472 & 196 & 5.20 \\
\hline Wyoming & $4,013,167$ & $8,636,000$ & 2.15 & 3,753 & 170 & 6.29 \\
\hline Total, 1933 & $333,630,533$ & $445,788,000$ & 1.34 & 418,703 & 167 & 4.78 \\
\hline Total, 1932 & $309,709,872$ & $406,677,000$ & 1.31 & 406,380 & 146 & 5.22 \\
\hline
\end{tabular}


TABLE XVI

Summary of Coal Produced, Value, Men Employed, Days Operated, and Output Per Man Per Day, by States, in 1934. (Exclusive of Product of Truck and Wagon Mines Producing Less Than One Thousand Tons)

\begin{tabular}{|c|c|c|c|c|c|c|}
\hline State & $\begin{array}{c}\text { Total } \\
\text { quantity }\end{array}$ & $\begin{array}{l}\text { Total } \\
\text { value }\end{array}$ & $\begin{array}{l}\text { Average } \\
\text { per ton }\end{array}$ & $\begin{array}{l}\text { Number of } \\
\text { employees }\end{array}$ & $\begin{array}{c}\text { Average number } \\
\text { of days mines } \\
\text { operated }\end{array}$ & $\begin{array}{l}\text { Average tons } \\
\text { per man per day }\end{array}$ \\
\hline Alabama & $9,142,117$ & $\$ 18,838,000$ & $\$ 2.06$ & 18,851 & 185 & 2.62 \\
\hline Alaska & 107,508 & 451,000 & 4.20 & 93 & 217 & 5.33 \\
\hline Arkansas & 856,432 & $2,564,000$ & 2.99 & 3,415 & 102 & 2.46 \\
\hline Oklahoma & $1,208,289$ & $2,846,000$ & 2.36 & 3,225 & 124 & 3.02 \\
\hline Colorado & $5,210,933$ & $12,309,000$ & 2.36 & 8,094 & 158 & 4.08 \\
\hline Illinois & $41,272,384$ & $64,238,000$ & 1.56 & 46,067 & 160 & 5.62 \\
\hline Indiana & $14,793,643$ & $21,838,000$ & 1.48 & 11,173 & 171 & 7.75 \\
\hline Iowa & $3,366,992$ & $7,862,000$ & 2.34 & 7,721 & 156 & 2.80 \\
\hline Kansas & $2,508,254$ & $4,619,000$ & 1.84 & 3,744 & 151 & 4.45 \\
\hline Missouri & $3,352,283$ & $6,278,000$ & 1.87 & 5,540 & 141 & 4.29 \\
\hline Kentucky & $38,525,235$ & $60,548,000$ & 1.57 & 49,509 & 180 & 4.33 \\
\hline Maryland & $1,627,112$ & $3,089,000$ & 1.90 & 2,976 & 176 & 3.12 \\
\hline Michigan & 621,741 & $1,940,000$ & 3.12 & 1,556 & 157 & 2.54 \\
\hline Montana & $2,565,702$ & $3,997,000$ & 1.56 & 1,590 & 166 & 9.73 \\
\hline New Mexico & $1,259,323$ & $3,402,000$ & 2.70 & 2,342 & 164 & 3.29 \\
\hline North Dakota & $1,753,888$ & $2,363,000$ & 1.35 & 1,518 & 174 & 6.65 \\
\hline South Dakota & 42,407 & 76,000 & 1.79 & 91 & 152 & 3.07 \\
\hline Ohio & $20,690,564$ & $34,774,000$ & 1.68 & 29,247 & 167 & 4.23 \\
\hline Pennsylvania & $89,825,875$ & $165,371,000$ & 1.84 & 126,079 & 179 & 3.98 \\
\hline Tennessee & $4,135,790$ & $7,514,000$ & 1.82 & 7,308 & 185 & 3.05 \\
\hline Georgia & 32,716 & 80,000 & 2.45 & 113 & 185 & 1.56 \\
\hline North Carolina & 3,140 & 9,000 & 2.87 & 18 & 221 & .79 \\
\hline Texas & 759,289 & $1,145,000$ & 1.51 & 805 & 178 & 5.30 \\
\hline Utah & $2,406,183$ & $4,746,000$ & 1.97 & 2,807 & 171 & 5.00 \\
\hline Virginia & $9,376,681$ & $16,375,000$ & 1.75 & 12,207 & 200 & 3.84 \\
\hline Washington & $1,382,991$ & $4,002,000$ & 2.89 & 2,161 & 193 & 3.32 \\
\hline West Virginia & $98,134,393$ & $167,104,000$ & 1.70 & 105,906 & 196 & 4.73. \\
\hline Wyoming & $4,367,961$ & $9,591,000$ & 2.20 & 3,760 & 188 & 6.17 \\
\hline Other States & 38,196 & 143,000 & 3.74 & 95 & 189 & 2.13 \\
\hline Total, 1934 & $359,368,022$ & $628,112,000$ & 1.75 & 458,011 & 178 & 4.40 \\
\hline Total, 1933 & $333,630,533$ & $445,788,000$ & 1.34 & 418,703 & 167 & 4.78 \\
\hline
\end{tabular}


TABLE XVII

Summary of Coal Produced, Value, Men Employed, Days Operated, and Output Per Man Per Day, by States, in 1935. (Exclusive of Product of Truck and Wagon Mines Producing Less Than One Thousand Tons)

\begin{tabular}{|c|c|c|c|c|c|c|}
\hline State & $\begin{array}{c}\text { Total } \\
\text { quantity }\end{array}$ & $\begin{array}{l}\text { Total } \\
\text { value }\end{array}$ & $\begin{array}{l}\text { Average } \\
\text { per tonl }\end{array}$ & $\begin{array}{c}\text { Total } \\
\text { number of } \\
\text { employees }\end{array}$ & $\begin{array}{c}\text { Average number } \\
\text { of days mines } \\
\text { operated }\end{array}$ & $\begin{array}{l}\text { Average tons } \\
\text { per man per day }\end{array}$ \\
\hline Alabama & $8,504,510$ & $\$ 18,251,000$ & $\$ 2.15$ & 18,906 & 161 & 2.79 \\
\hline Alaska & 119,425 & 502,000 & 4.20 & 95 & 249 & 5.05 \\
\hline Arizona, California, Idaho, and Oregon & 24,844 & 95,000 & 3.82 & 103 & 140 & 1.72 \\
\hline Arkansas & $1,133,279$ & $3,448,000$ & 3.04 & 3,743 & 123 & 2.47 \\
\hline Colorado & $5,910,511$ & $13,675,000$ & 2.31 & 8,153 & 177 & 4.08 \\
\hline Georgia and North Carolina & 22,734 & 58,000 & 2.55 & 109 & 160 & 1.30 \\
\hline Illinois & $44,525,469$ & $69,516,000$ & 1.56 & 43,748 & 171 & 5.97 \\
\hline Indiana & $15,754,214$ & $23,722,000$ & 1.51 & 11,347 & 176 & 7.91 \\
\hline Iowa & $3,650,163$ & $9,002,000$ & 2.47 & 8,038 & 162 & 2.80 \\
\hline Kansas & $2,686,164$ & $4,943,000$ & 1.84 & 3,896 & 173 & 4.00 \\
\hline Kentucky & $40,760,939$ & $65,956,000$ & 1.62 & 52,339 & 182 & 4.28 \\
\hline Maryland & $1,678,059$ & $3,266,000$ & 1.95 & 2,962 & 179 & 3.17 \\
\hline Michigan & 628,384 & $2,017,000$ & 3.21 & 1,467 & 158 & 2.70 \\
\hline Missouri & $3,645,996$ & $6,924,000$ & 1.90 & 5,710 & 159 & 4.02 \\
\hline Montana - & $2,758,906$ & $4,146,000$ & 1.50 & 1,571 & 189 & 9.30 \\
\hline New Mexico & $1,388,877$ & $3,681,000$ & 2.65 & 2,355 & 185 & 3.19 \\
\hline North Dakota & $1,955,510$ & $2,395,000$ & 1.22 & 1,365 & 188 & 7.61 \\
\hline Ohio & $21,153,151$ & $35,111,000$ & 1.66 & 29,524 & 162 & 4.44 \\
\hline Oklahoma & $1,229,398$ & $2,879,000$ & 2.34 & 3,151 & 122 & 3.19 \\
\hline Pennsylvania & $91,404,670$ & $172,170,000$ & 1.88 & 124,109 & 180 & 4.10 \\
\hline South Dakota & 13,243 & 21,000 & 1.59 & 55 & 98 & 2.46 \\
\hline Tennessee & $4,137,802$ & $7,435,000$ & 1.80 & 7,531 & 181 & 3.04 \\
\hline Texas & 757,529 & 654,000 & .86 & 792 & 177 & 5.42 \\
\hline Utah & $2,946,918$ & $6,091,000$ & 2.07 & 2,752 & 188 & 5.70 \\
\hline Virginia & $9,667,018$ & $17,128,000$ & 1.77 & 13,043 & 189 & 3.92 \\
\hline Washington & $1,559,206$ & $4,686,000$ & 3.01 & 2,258 & 192 & 3.60 \\
\hline West Virginia & $99,179,061$ & $169,164,000$ & 1.71 & 109,315 & 192 & 4.74 \\
\hline Wyoming & $5,177,142$ & $11,127,000$ & 2.15 & 3,966 & 217 & 6.00 \\
\hline Total, $19 \overline{35}$ & $372,373,122$ & $658,063,000$ & 1.77 & 462,403 & 179 & 4.50 \\
\hline Total, 1934 & $359,368,022$ & $628,383,000$ & 1.75 & 458,011 & 178 & 4.40 \\
\hline
\end{tabular}


TABLE XVIII

Summary of Coal Produced. Men Employed, Days Operated; and Output Per Man Per Day, by States, in 1936. (Exclusive of Product of Truck and Wagon Mines Producing Less Than One Thousand Tons)

\begin{tabular}{|c|c|c|c|c|}
\hline State & $\begin{array}{c}\text { Total } \\
\text { quantity }\end{array}$ & $\begin{array}{c}\text { Total } \\
\text { number of } \\
\text { employees }\end{array}$ & $\begin{array}{l}\text { Average num- } \\
\text { ber of days } \\
\text { mines operated }\end{array}$ & $\begin{array}{c}\text { Average } \\
\text { tons per man } \\
\text { per day }\end{array}$ \\
\hline Alabama & $12,229,287$ & $2 \overline{0,491}$ & 206 & 2.90 \\
\hline Alaska & 136,593 & 111 & 245 & 5.02 \\
\hline Arizona, Idaho, and Oregon & 15,364 & 53 & 202 & 1.43 \\
\hline Arkansas & $1,622,787$ & 4,123 & 152 & 2.58 \\
\hline Colorado & $6,811,802$ & 8,802 & 191 & 4.06 \\
\hline Georgia & 24,288 & 94 & 207 & 1.25 \\
\hline Illinois & $50,926,599$ & 44,347 & 175 & 6.55 \\
\hline Indiana & $17,822,536$ & 11,801 & 178 & 8.48 \\
\hline Iowa & $3,960,700$ & 8,741 & 163 & 2.78 \\
\hline Kansas & $2,944,028$ & 3,755 & 163 & 4.80 \\
\hline Kentucky & $47,521,950$ & 54,089 & 202 & 4.34 \\
\hline Maryland & $1,703,589$ & 2,916 & 186 & 3.14 \\
\hline Michigan & 626,145 & 1,400 & 164 & 2.73 \\
\hline Missouri & $3,984,999$ & 5,654 & 171 & 4.13 \\
\hline Montana 1 & $2,988,524$ & 1,459 & 195 & 10.53 \\
\hline New Mexico & $1,596,775$ & 2,392 & 202 & 3.30 \\
\hline North Dakota ${ }^{1}$ & $2,215,335$ & 1,408 & 192 & 8.20 \\
\hline Ohio & $24,110,078$ & 29,853 & 183 & 4.42 \\
\hline Oklahoma & $1 ; 540,303$ & 3,153 & 155 & 3.15 \\
\hline Pennsylvania & $109,887,470$ & 127,211 & 205 & 4.22 \\
\hline South Dakota 1 & $\begin{array}{r}41,331 \\
\end{array}$ & 50 & 231 & 3.58 \\
\hline Tennessee & $5,108,195$ & 7,982 & 203 & 3.16 \\
\hline Texas ${ }^{1}$ & 842,624 & 810 & 192 & 5.42 \\
\hline Utah & $3,246,565$ & 3,057 & 186 & 5.70 \\
\hline Virginia & $11,661,636$ & 14,882 & 196 & 4.00 \\
\hline Washington & $1,812,104$ & 2,625 & 200 & 3.46 \\
\hline West Virginia & $117,925,706$ & 111,468 & 216 & 4.89 \\
\hline Wyoming & $5,780,590$ & 4,477 & 215 & 6.00 \\
\hline Total, 1936 & $439,087,903$ & 477,204 & 199 & 4.62 \\
\hline Total, 1935 & $372,373,122$ & 462,403 & 179 & 4.50 \\
\hline
\end{tabular}

1 Includes figures on lignite compiled by Bureau of Mines. 
TABLE, XIX

Summary of Coal Produced, Men Employed, Days Operated, and Output Per Man Per Day, by States, in 1937. (Exclusive of Product of. Truck and Wagon Mines Producing Less Than One Thousand Tons)

\begin{tabular}{|c|c|c|c|c|}
\hline State & $\begin{array}{c}\text { Total } \\
\text { quantity }\end{array}$ & $\begin{array}{l}\text { Average } \\
\text { number of } \\
\text { employees }\end{array}$ & $\begin{array}{l}\text { Average num- } \\
\text { ber of days } \\
\text { mines operated }\end{array}$ & $\begin{array}{c}\text { Average } \\
\text { tons per man } \\
\text { per day } \\
\end{array}$ \\
\hline Alabama & $11,760,221$ & 22,613 & 200 & 2.74 \\
\hline Abaska & 128,608 & 123 & 207 & 5.16 \\
\hline Arkansas & $1,465,468$ & 4,253 & 136 & 2.61 \\
\hline Colorado & $5,338,492$ & 9,432 & 181 & 4.21 \\
\hline Illinois & $42,824,674$ & 42,449 & 168 & 7.25 \\
\hline Indiana & $15,546,298$ & 11,238 & 174 & 9.10 \\
\hline Iowa & $1,731,173$ & 8,720 & 146 & 2.87 \\
\hline Kansas & $2,520,166$ & 3,574 & 173 & 4.68 \\
\hline Kentucky & $45,216,686$ & 55,596 & • 192 & 4.41 \\
\hline Maryland & $1,283,125$ & 2,525 & 189 & 3.25 \\
\hline Michigan & 180,969 & 1,343 & 145 & 2.88 \\
\hline Missouri & $2,950,424$ & 6,436 & 152 & 4.18 \\
\hline Montana ${ }^{1}$ & $2,767,758$ & 1,503 & 186 & 10.62 \\
\hline New Mexico & $1,552,612$ & 2,608 & 208 & 3.17 \\
\hline North Dakota 1 & $1,577,216$ & 1,475 & 181 & 8.41 \\
\hline Ohio & $20,792,181$ & 30,294 & 185 & 4.49 \\
\hline Oklahoma & $1,411,648$ & 3,147 & 142 & 3.58 \\
\hline Pennsylvania & $96,758,008$ & 133,897 & 199 & 4.16 \\
\hline South Dakota I & 26,444 & 47 & 165 & 6.05 \\
\hline Tennessee & $4,812,564$ & 8,465 & 195 & 3.17 \\
\hline Texas ${ }^{1}$ & 861,793 & 819 & 199 & 5.59 \\
\hline Utah & $3,500,403$ & 3,417 & 189 & 5.88 \\
\hline Virginia & $13,017,015$ & 16,494 & 200 & 4.18 \\
\hline Washington & $1,445,024$ & 2,882 & 204 & 3.40 \\
\hline West Virginia & $114,227,504$ & 113,643 & 209 & 5.00 \\
\hline Wyoming & $5,530,230$ & 4,743 & 204 & 6.11 \\
\hline Other States ${ }^{2}$ & 10,871 & 128 & 165 & 1.15 \\
\hline Total, 1937 & $399,237,575$ & 491,864 & 193 & 4.69 \\
\hline Total, 1936 & $439,087,903$ & 477,204 & 199 & 4.62 \\
\hline
\end{tabular}

1 Includes figures on lignite from Bureau of Mines; "Loaded at mines for shipment," as published by Bureau of Mines, included under "all-rail"; "commercial sales by truck or wagon" and "other sales to local trade, etc.," as published by Bureau of Mines, included under "truck deliveries including local sales."

2 Arizona, California, Georgia, Idaho, and Oregon. 


\section{Gypsum}

Gypsum clepends for markets almost entirely upon building construction and particularly residential building. It is natural, therefore, that when building construction activities dropped off after 1929 one result was a decline in production and value of gypsum.

Most of the calcined gypsum produced is used to make building materials such as plasters, wallboards, partition tile, and insulating materials, and smaller quantities are sold to manufacturing companies for special purposes. Uncalcined or raw gypsum is used chiefly by the cement industry as a retarder, although land plaster and other outlets are important at times.

Raw or crude gypsum is not and has never been a high-priced product and in recent years there has been a tendency among the producers of raw gypsum to manufacture and attempt to increase the markets for gypsum products rather than to increase sales of the crude material.

Nationally, Iowa ranks third in the value and production of gypsum and is outranked only by New York and Michigan in the order named. The entire industry in the State has been centered in the immediate vicinity of Fort Dodge, Webster county since 1934. Prior to that rime a minor percentage of the total production was obtained from Appanoose county.

Gypsum followed the general trend of mineral production in Iowa, but was somewhat above the average during the period of this report. The tonnage of crude gypsum mined dropped off in 1933 but increased each year since then except during 1938. The largest increase came in 1936 when it amounted to approximately 50 per cent over 1935 and was greater than any year since the beginning of the depression.

Sales of uncalcined gypsum decreased in tonnage and total value in 1933 and in 1935, recovered in 1934 and increased greatly in 1936, the last year on which data were collected. The value and tonnage of "calcined gypsum sold" fell off in 1933 but showed increases in 1934, 1935 and 1936.

Table XX shows production figures for Iowa for the period 1932 1938. A revised method of canvassing the gypsum industry was begun in 1937 by the Bureau of Mines at the request of the producing companies. By this revision no data were collected by states after 1936 on gypsum "sold calcined" and "sold uncalcined". This has a bearing 
TABLE XX

Production of Gypsum in Iowa from 1932 to 1938

\begin{tabular}{|c|c|c|c|c|c|c|c|c|}
\hline \multirow[b]{2}{*}{ Year } & \multirow[b]{2}{*}{$\begin{array}{l}\text { Active } \\
\text { mines }\end{array}$} & \multicolumn{2}{|c|}{ Crude gypsum mined } & \multicolumn{2}{|c|}{ Sold uncalcined } & \multicolumn{2}{|c|}{ Sold calcined } & \multirow[b]{2}{*}{$\begin{array}{l}\text { Total } \\
\text { value }\end{array}$} \\
\hline & & $\begin{array}{c}\text { Short } \\
\text { tons }\end{array}$ & Value & $\begin{array}{l}\text { Short } \\
\text { tons }\end{array}$ & Value & $\begin{array}{l}\text { Short } \\
\text { tons }\end{array}$ & Value & \\
\hline 1932 & 7 & 178,087 & & 63,931 & $\overline{\$ 91,267}$ & 105,788 & $\$ 1,377,147$ & $\$ 1,468,414$ \\
\hline 1933 & 8 & 172,555 & & 58.863 & 75,083 & 104.371 & $1,282.324$ & 1,357407 \\
\hline 1934 & 8 & 180,271 & & 63,510 & 97,626 & 115,282 & $1,572,730$ & $1,670,356$ \\
\hline 1935 & 7 & 230,203 & $\$ 233,926$ & 54,150 & 74,914 & 151,366 & $2,109,010$ & $2,215,770^{\mathrm{a}}$ \\
\hline 1936 & 8 & 344,221 & 352,834 & 97,511 & 146,233 & 217,088 & $3,115,155$ & $3,261,388$ \\
\hline 1937 & 8 & 387,255 & 533,162 & (b) & (b) & (b) & (b) & (b) \\
\hline 1938 & 8 & 364,920 & 495,856 & (b) & (b) & (b) & (b) & (b) \\
\hline
\end{tabular}

a Revised

b Data not collected.

on "total value" recorded in the tables including gypsum in Iowa, in that the value of crude gypsum is substituted for total value of the calcined and uncalcined products. The total value of crude is much less than the combined value of calcined and uncalcined gypsum, and in Table $I$ this makes it appear that total values in 1937 and 1938 were far below average, whereas these two years were actually well above the average of the period of this report. Other changes affect the figures for the United States "total value" and "calcined gypsum products sold".

The drastic decrease in production of gypsum in the United States which began in 1930 continued until 1933 and the amount produced in 1933 was less than in any year since 1905. The price dropped an additional 6 per cent in 1933, but late in that year the market started an upswing. In 1934 the total production increased 15 per cent, but was still below the general recovery level because of the lag of residential building. Increases in production of both raw and calcined gypsum continued through 1935, and in 1936 the crude product increased an additional 42 per cent and the sales of gypsum products increased 29 per cent over 1935. The advance was due principally to increase in privately financed building construction. Further increases continued through the early months of 1937 and the total value of uncalcined and calcined gypsum for 1937 exceeded that for 1936, but production fell off during the last half of the year. Activity continued to be slower through much of 1938 but in the last quarter improvement was definitely under way.

Table XXI shows production of gypsum in the United States for the period of this report.

During 1937 products derived from byproduct gypsum (first util- 
TABLE XXI

Production of Gypsum in the United States from 1932 to 1938

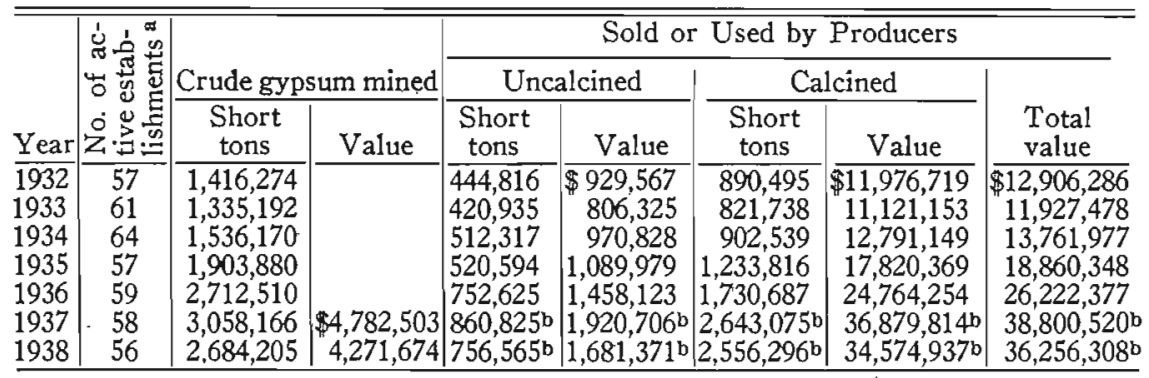

a Each mine, plant or combination mine plant is counted as one establishment; beginning in 1937 plants utilizing by-product gypsum are included.

b Includes gypsum products made from domestic, imported, and by-product crude gypsum sold or used in the United States.

ized about 1925) comprised an appreciable part of the total sales value, and the process for a new product, hydraulic gypsum was patented. In 1938 two new processing plants were opened, one in Georgia and one in Florida.

\section{Webster County}

\section{Gypsum Producers in Iowa}

Cardiff Gypsum Co., 903 Central Avenue, Fort Dodge, Iowa. Mine and mill at Gypsum, Iowa.

Certain-teed Products Corporation, Att: E. H. Laney, Assistant Auditor, 100 E. 42nd Street, New York, New York. Mill and mine at Fort Dodge.

A. R. Eno, Fort Dodge, Iowa.

Fort Dodge Plaster Co., J. M. Norton, Manager, 1420 Seventh Avenue, North, Fort Dodge, Iowa. Mine at Fort Dodge.

Hawkeye Gypsum Products Co., John E. Gustin, Manager, Fort Dodge, Iowa. Mine at Fort Dodge.

Johnston Clay Works, Inc., 214 First National Bank Building, Fort Dodge, Iowa. Mine at Fort Dodge.

National Gypsum Co., F. E. Davis, Treasurer, 192 Deleware Avenue, Buffalo, New York. Mill and mine at Fort Dodge.

United States Gypsum Co., 300 West Adams Street, Chicago, Illinois. Mill and mine at Fort Dodge.

Wasem Plaster Co., H. W. Wasem, President, Warden Building, Fort Dodge, Iowa. Mill and mine at Fort Dodge.

\section{Limestone}

Limestone is utilized chiefly for road metal and concrete and these 
uses generally govern the trends of production. Important secondary uses in Iowa are for agricultural purposes and riprap, and during the past few years an increasing amount has been consumed by sugar factories.

The limestone industry had a remarkable history in Iowa during the period 1933-1938. The low of 1933 was well above the average of total production from 1895 to 1925 . In 1934 all previous records were broken and although there was a decline in 1935, that year was sixth in rank for the years after 1895. All records were again broken in 1936 when total quantity and total value more than doubled the figures of the previous year. The peak was reached in 1937 when the total quantity produced was $4,272,670$ tons valued at $\$ 4,261,184$ which was more than four times greater than for 1933. Production declined in 1938, but exceeded that of any earlier year except 1937 and the average price per ton was higher than for any year during the period of this report.

Production of limestone for road metal and concrete after a drop in 1933, rallied strongly in 1934 and decreased slightly in 1935. In 1936 there was an increase of more than 100 per cent. Records in total tonnage and value were established in 1937, and in 1938 the advance in prices permitted only a slight decline in value in spite of a sharp drop in quantity produced.

Limestone used for agriculture followed approximately the same trends as that used for road metal and concrete. Riprap had a similar history although the variations in productions and values were more spectacular. Building stone, rubble and flux varied greatly; building stone showed exceptionally high production in 1937, while rubble production was considerably above average in 1933.

It is significant that the number of producers in Iowa increased steadily from 60 in 1933 to 174 in 1937 . and that the noncommercial operators and production became proportionally larger each year. The drop in the number of producers in 1938 was due to the increased activity of W. P. A., considered as one operator, which reported an output of $1,739,625$ tons valued at $\$ 2,392,875$, representing 52 per cent of total production and 64 per cent of total value for the year.

Statistics of limestone production in Iowa may be found in Table XXII and more detailed data by counties in Tables XXIV to XXIX. In the latter tables it was necessary to combine counties in order to avoid revealing confidential information. 
TABLE, XXII

Production of Limestone in Iowa from 1932 to 1938

\begin{tabular}{|c|c|c|c|c|c|c|c|c|c|}
\hline & $\begin{array}{l}\text { Building } \\
\text { stone }\end{array}$ & Rubble & Flux & Rip rap & $\begin{array}{c}\text { Road metal } \\
\text { and } \\
\text { concrete }\end{array}$ & $\begin{array}{c}\text { Railroad } \\
\text { ballast }\end{array}$ & $\begin{array}{l}\text { Agri- } \\
\text { culture }\end{array}$ & $\begin{array}{c}\text { Other } \\
\text { limestone }\end{array}$ & Total \\
\hline 1932 & & & & & & & & & \\
\hline $\begin{array}{l}\text { Short tons } \\
\text { Value }\end{array}$ & $\begin{array}{r}1,672 \\
\$ 1,929\end{array}$ & $\begin{array}{l}2,329 b \\
3,088^{b}\end{array}$ & & $\begin{array}{l}23,686 \\
19,069\end{array}$ & $\begin{array}{l}1,475,718^{c} \\
1,283,713^{c}\end{array}$ & & $\begin{array}{l}67,663 \\
50,983\end{array}$ & $\begin{array}{l}20,167 \\
30,683\end{array}$ & $\begin{array}{l}1,591,235 \\
1,389,465\end{array}$ \\
\hline $\begin{array}{l}\text { Producers } \\
1933\end{array}$ & 4 & 2 & 1 & & $47 \mathrm{c}$ & 1 & 24 & 4 & $53^{\mathrm{J}}$ \\
\hline $\begin{array}{l}\text { Short tons } \\
\text { Value }\end{array}$ & $\begin{array}{r}4,270 \\
\$ 4,402\end{array}$ & $\begin{array}{r}11,620 \\
9,484\end{array}$ & $\begin{array}{l}3,476 \\
3,889\end{array}$ & $\begin{array}{l}9,690 \\
8,830\end{array}$ & $\begin{array}{l}861,550 \\
781,750\end{array}$ & $\begin{array}{l}46,640 \\
21,485\end{array}$ & $\begin{array}{l}63,610 \\
44,649\end{array}$ & $\begin{array}{l}30,440^{d} \\
34,703^{d}\end{array}$ & $\begin{array}{c}1,031,290^{\mathrm{k}} \\
909,192\end{array}$ \\
\hline $\begin{array}{l}\text { Producers } \\
1934\end{array}$ & 3 & 3 & 2 & 9 & 51 & & 27 & & $60^{\mathrm{s}}$ \\
\hline $\begin{array}{l}\text { Short tons } \\
\text { Value }\end{array}$ & & $\begin{array}{r}1,870 \\
\$ 1,752\end{array}$ & & $\begin{array}{l}67,220 \\
48,285\end{array}$ & $\begin{array}{l}2,035,900^{c} \\
1,734,123^{c}\end{array}$ & & $\begin{array}{r}143,386 \\
96,164\end{array}$ & $\begin{array}{l}22,560^{\mathrm{e}} \\
49,086^{\mathrm{e}}\end{array}$ & $\begin{array}{l}2,276,440^{\mathrm{k}} \\
1,934,364\end{array}$ \\
\hline $\begin{array}{l}\text { Producers } \\
1935\end{array}$ & 2 & 3 & 2 & 14 & 95 & 2 & 24 & $7 e$ & $101^{\mathrm{J}}$ \\
\hline $\begin{array}{l}\text { Short tons } \\
\text { Value }\end{array}$ & $\begin{array}{r}4,070 \\
\$ 3,685\end{array}$ & $\begin{array}{l}1,790 \\
1,623\end{array}$ & & $\begin{array}{l}53,220 \\
44,267\end{array}$ & $\begin{array}{l}1,642,080 \mathrm{c} \\
1,447,993^{c}\end{array}$ & & $\begin{array}{r}104,080 \\
82,107\end{array}$ & $\begin{array}{l}33,840^{r} \\
67,763^{r}\end{array}$ & $\begin{array}{l}1,840,080^{\mathrm{k}} \\
1,645,937\end{array}$ \\
\hline $\begin{array}{l}\text { Producers } \\
1936\end{array}$ & & 4 & 2 & 12 & $103 \mathrm{c}$ & 1 & & & 105 \\
\hline $\begin{array}{l}\text { Short tons } \\
\text { Value }\end{array}$ & & $\begin{array}{r}1,916 \\
\$ 1,887\end{array}$ & $\begin{array}{l}11,457 \\
12,070\end{array}$ & $\begin{array}{l}120,230 \\
139,774\end{array}$ & $\begin{array}{l}3,521,170 \\
2,943,060\end{array}$ & $\begin{array}{r}107,122 \\
48,233\end{array}$ & $\begin{array}{l}222,700 \\
197,688\end{array}$ & $\begin{array}{l}18,950 \mathrm{~g} \\
54,543 \mathrm{~g}\end{array}$ & $\begin{array}{l}4,003,550^{k} \\
3,397,356\end{array}$ \\
\hline $\begin{array}{l}\text { Producers } \\
1937\end{array}$ & 4 & & 2 & 11 & 118 & 3 & 27 & $7 \mathrm{~g}$ & $130^{\jmath}$ \\
\hline $\begin{array}{l}\text { Short tons } \\
\text { Value }\end{array}$ & $\begin{array}{r}13,830 \\
\$ 12,570\end{array}$ & & $\begin{array}{l}16,790 \\
17,279\end{array}$ & $\begin{array}{l}260,290 \\
193,392\end{array}$ & $\begin{array}{l}3,621,540 \\
3,662,185\end{array}$ & $\begin{array}{l}39,690 \\
20,881\end{array}$ & $\begin{array}{l}304,690 \\
301,845\end{array}$ & $\begin{array}{r}8,870^{\mathbf{h}} \\
41,298^{\mathrm{h}}\end{array}$ & $\begin{array}{l}4,272,670 \\
4,261,184\end{array}$ \\
\hline Producers & 4 & 1 & 3 & 31 & 173 & 4 & 58 & $6^{h}$ & 174 \\
\hline $\begin{array}{l}1938 \\
\text { Short tons }\end{array}$ & 2,410 & & & 182,180 & $2,853,890^{c}$ & & 236,300 & $34,000^{I}$ & 3.323750 \\
\hline Value & $\$ 1,935$ & & & 113,089 & $3,313,323^{c}$ & & 207,883 & $78,013^{1}$ & $3,742,580$ \\
\hline Producers & 4 & 3 & 2 & 18 & 69 & 2 & 46 & 171 & $85^{j}$ \\
\hline \multicolumn{10}{|c|}{$\begin{array}{l}\text { a Chiefly rough construction. } \\
\text { b Includes fux. } \\
\text { c Includes railroad ballast. } \\
\text { d Includes that sold to sugar factories. } \\
\text { e Includes building stone, curbing and flux. } \\
\text { f Includes curbing, fux. }\end{array}$} \\
\hline
\end{tabular}


In the United States as a whole, as in lowa, the most important uses of limestone are for road metal and concrete. Following in the order of importance are uses for fluxing, argriculture, railroad ballast and riprap. Their relative importance and other details of production in the United States may be found in Table XXIII in which limestone utilized for cement manufacture is not included. The table shows that total production and total value of limestone decreased in 1933, increased in 1934, decreased slightly in 1935, increased materially in 1936 and 1937 and again decreased in 1938.

In 1933 the value of limestone for all uses increased over the nation except for concrete and road metal which fell off about 6.4 millions of dollars, but in 1934 the values for all uses increased; that for road metal and concrete advanced approximately 8.5 millions of dollars. All values again increased in 1935 except for road metal and concrete, riprap and railroad ballast. In 1936 decreases occured only in limestone used for building stone and rubble, and these clecreases were easily offset by increases in other fieldsi which brought about an advance of approximately 60 per cent in total value of all limestone produced. Further increase in 1937 in all items except rubble, riprap and railroad ballast resulted in the largest total production and total value for the period of this report. As in other mineral industries production of limestone in the United States fell off in 1938 except that used for road metal and concrete, rubble, and riprap. 
TABLE XXXIII

Production of Limestone in the United States from 1932 to 1938

\begin{tabular}{|c|c|c|c|c|c|c|c|c|}
\hline & $\begin{array}{l}\text { Building } \\
\text { stone } \mathbf{a}\end{array}$ & Rubble & Flux & Rip rap & $\begin{array}{c}\text { Concrete } \\
\text { and road } \\
\text { metal }\end{array}$ & $\begin{array}{c}\text { Railroad } \\
\text { ballast }\end{array}$ & $\begin{array}{c}\text { Agri- } \\
\text { culture }\end{array}$ & Total b \\
\hline $\begin{array}{l}1932 \\
\text { Short tons } \\
\text { Value } \\
1933\end{array}$ & $\begin{array}{r}50,580 \\
\$ 77,872\end{array}$ & $\begin{array}{l}84,570 \\
84,308\end{array}$ & $\begin{array}{l}3,945,170 \\
2,902,847\end{array}$ & $\begin{array}{l}1,448,040 \\
1,421,024\end{array}$ & $\begin{array}{l}32,612,550 \\
28,650,198\end{array}$ & $\begin{array}{l}2,450,970 \\
1,748,412\end{array}$ & $\begin{array}{r}909,470 \\
1,229,107\end{array}$ & $\begin{array}{l}46,913,520 \\
48,015,748\end{array}$ \\
\hline $\begin{array}{l}\text { Short tons } \\
\text { Value } \\
1934\end{array}$ & $\begin{array}{r}78,790 \\
\$ 108,100\end{array}$ & $\begin{array}{l}79,060 \\
94,046\end{array}$ & $\begin{array}{l}7,982,560 \\
5,510,445\end{array}$ & $\begin{array}{l}1,566,560 \\
1,767,541\end{array}$ & $\begin{array}{l}25,820,640 \\
22,239,698\end{array}$ & $\begin{array}{l}2,786,050 \\
1,896,308\end{array}$ & $\begin{array}{r}994,540 \\
1,239,724\end{array}$ & $\begin{array}{l}45,922,280 \\
44,499,311\end{array}$ \\
\hline $\begin{array}{l}\text { Short tons } \\
\text { Value } \\
1935\end{array}$ & $\begin{array}{r}156,000 \\
\$ 179,000\end{array}$ & $\begin{array}{l}190,080 \\
179,791\end{array}$ & $\begin{array}{l}9,230,880 \\
6,297,579\end{array}$ & $\begin{array}{l}2,490,760 \\
2,668,215\end{array}$ & $\begin{array}{l}33,209,910 \\
30,749,136\end{array}$ & $\begin{array}{l}3,614,430 \\
2,549,091\end{array}$ & $\begin{array}{l}1,612,380 \\
1,788,142\end{array}$ & $\begin{array}{l}57,501,510 \\
53,790,846\end{array}$ \\
\hline $\begin{array}{l}\text { Short tons } \\
\text { Value } \\
1936\end{array}$ & $\begin{array}{r}293,050 \\
\$ 310,878\end{array}$ & $\begin{array}{l}185,790 \\
276,569\end{array}$ & $\begin{array}{r}12,191,660 \\
7,902,717\end{array}$ & $\begin{array}{l}1,982,250 \\
1,890,625\end{array}$ & $\begin{array}{l}30,151,790 \\
26,354,559\end{array}$ & $\begin{array}{l}3,623,500 \\
2,525,949\end{array}$ & $\begin{array}{l}2,140,370 \\
2,656,728\end{array}$ & $\begin{array}{l}57,492,760 \\
50,668,765\end{array}$ \\
\hline $\begin{array}{l}\text { Short tons } \\
\text { Value } \\
1937\end{array}$ & $\begin{array}{r}156,970 \\
\$ 272,164\end{array}$ & $\begin{array}{l}204,700 \\
181,415\end{array}$ & $\begin{array}{l}17,724,880 \\
11,576,156\end{array}$ & $\begin{array}{l}2,205,700 \\
3,275,193\end{array}$ & $\begin{array}{l}49,751,570 \\
46,058,424\end{array}$ & $\begin{array}{l}5,101,580 \\
3,632,649\end{array}$ & $\begin{array}{l}3,907,710 \\
4,512,703\end{array}$ & $\begin{array}{l}87,735,740 \\
81,559,984\end{array}$ \\
\hline $\begin{array}{l}\text { Short tons } \\
\text { Value } \\
1938\end{array}$ & $\begin{array}{r}191,660 \\
\$ 380,324\end{array}$ & $\begin{array}{l}107,550 \\
136,028\end{array}$ & $\begin{array}{l}21,311,250 \\
14,685,215\end{array}$ & $\begin{array}{l}2,769,640 \\
2,891,936\end{array}$ & $\begin{array}{l}51,108,620 \\
49,547,350\end{array}$ & $\begin{array}{l}5,033,180 \\
3,588,974\end{array}$ & $\begin{array}{l}5,004,930 \\
6,454,695\end{array}$ & $\begin{array}{l}94,577,270 \\
90,901,877\end{array}$ \\
\hline $\begin{array}{l}\text { Short tons } \\
\text { Value }\end{array}$ & $\begin{array}{r}166,260 \\
\$ 316,772 \\
\end{array}$ & $\begin{array}{l}155,370 \\
194,621\end{array}$ & $\begin{array}{l}9,692,130 \\
6,933,621\end{array}$ & $\begin{array}{l}2,590,770 \\
3,107,511\end{array}$ & $\begin{array}{l}54,357,130 \\
52,387,376\end{array}$ & $\begin{array}{l}3,187,770 \\
2,210,881\end{array}$ & $\begin{array}{l}4,367,410 \\
5,637,485\end{array}$ & $\begin{array}{l}81,679,690 \\
82,286,555\end{array}$ \\
\hline
\end{tabular}

a Rough construction except as noted.
b Including rough architectural, finished building stone, curbing, flagging, paving, stone used in sugar factories, glass factories and paper mills and other uses. 
TABLE XXIV

Production of Limestone in Iozva in 1933

\begin{tabular}{|c|c|c|c|c|c|c|c|}
\hline \multirow[b]{2}{*}{ Counties } & \multirow[b]{2}{*}{$\begin{array}{l}\text { Number of } \\
\text { producers a }\end{array}$} & \multicolumn{2}{|c|}{$\begin{array}{l}\text { Road metal, concrete, } \\
\text { railroad ballast }\end{array}$} & \multicolumn{2}{|c|}{ Other uses b } & \multicolumn{2}{|c|}{ Total } \\
\hline & & $\begin{array}{l}\text { Short } \\
\text { tons }\end{array}$ & Value & $\begin{array}{l}\text { Short } \\
\text { tons }\end{array}$ & Value & $\begin{array}{l}\text { Short } \\
\text { tons }\end{array}$ & Value \\
\hline $\begin{array}{l}\text { Allamakee (1), Clayton (1), } \\
\text { Delaware (2) }\end{array}$ & 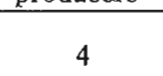 & 45,771 & $\$ 33,054$ & (c) & (c) & 45,771 & $\$ 33,054$ \\
\hline $\begin{array}{l}\text { Black Hawk (3), Bremer (1), } \\
\text { Cerro Gordo (1). Floyd (1) }\end{array}$ & 6 & 62787 & 84.462 & 22.965 & $\$ 32,708$ & 85.752 & 117170 \\
\hline Buchanan (1), Johnson (2), Linn (5) & 8 & $\begin{array}{r}02,7 \% \\
122,875\end{array}$ & 115,742 & $\begin{array}{r}22,905 \\
7,600\end{array}$ & 6,807 & 130,475 & 122,549 \\
\hline Dubuque (3), Jackson (2), Jones (5) & 10 & 164,815 & $\begin{array}{r}166,179 \\
64275\end{array}$ & 30,542 & $\begin{array}{l}22,900 \\
\text { (c) }\end{array}$ & $\begin{array}{r}195,357 \\
63,620\end{array}$ & $\begin{array}{r}189.079 \\
64.275\end{array}$ \\
\hline Scott (3), Louisa (1) & $\begin{array}{l}5 \\
4\end{array}$ & $\begin{array}{r}63,020 \\
135,020\end{array}$ & 94,275 & 17,514 & 12,696 & 152,534 & 109,248 \\
\hline $\begin{array}{l}\text { Davis }(2) \text {, Keokuk (1), Lee (3), } \\
\text { Mahaska (1), Van Buren (2), } \\
\text { Wapello (1), Wayne (1) }\end{array}$ & 11 & 128,277 & 106,425 & (c) & (c) & 128,277 & 106,425 \\
\hline $\begin{array}{l}\text { Grundy (1), Hardin (1), Madison (3), } \\
\text { Marshall (2), Tama (1) }\end{array}$ & 8 & 161,488 & 125,313 & 24,909 & 17,189 & 186,397 & 142,502 \\
\hline $\begin{array}{c}\text { Fremont (1), Montgomery (1), } \\
\text { Woodbury }(2) \\
\text { Total, } 1933 \\
\text { Total, } 1932\end{array}$ & $\begin{array}{r}4 \\
60 \\
54\end{array}$ & $\begin{array}{r}43,099 \\
927,752 \\
1,491,861\end{array}$ & $\begin{array}{r}24,890 \\
\$ 816,892 \\
1,295,039\end{array}$ & $\begin{array}{c}(c) \\
103,530 \\
89,005\end{array}$ & $\begin{array}{c}(c) \\
\$ 92,300 \\
83,583\end{array}$ & 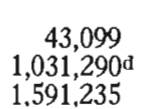 & $\begin{array}{r}24,890 \\
\$ 909,192 \\
1,389,465\end{array}$ \\
\hline
\end{tabular}

a Commercial and noncommercial.

b Other uses includes: Agricultural, sugar factories, building stone, rubble, riprap, flux, poultry grit.

c Included under road metal, concrete and railroad ballast for purposes of 
TABLE XXV

Production of Limestone in Iowa in 1934

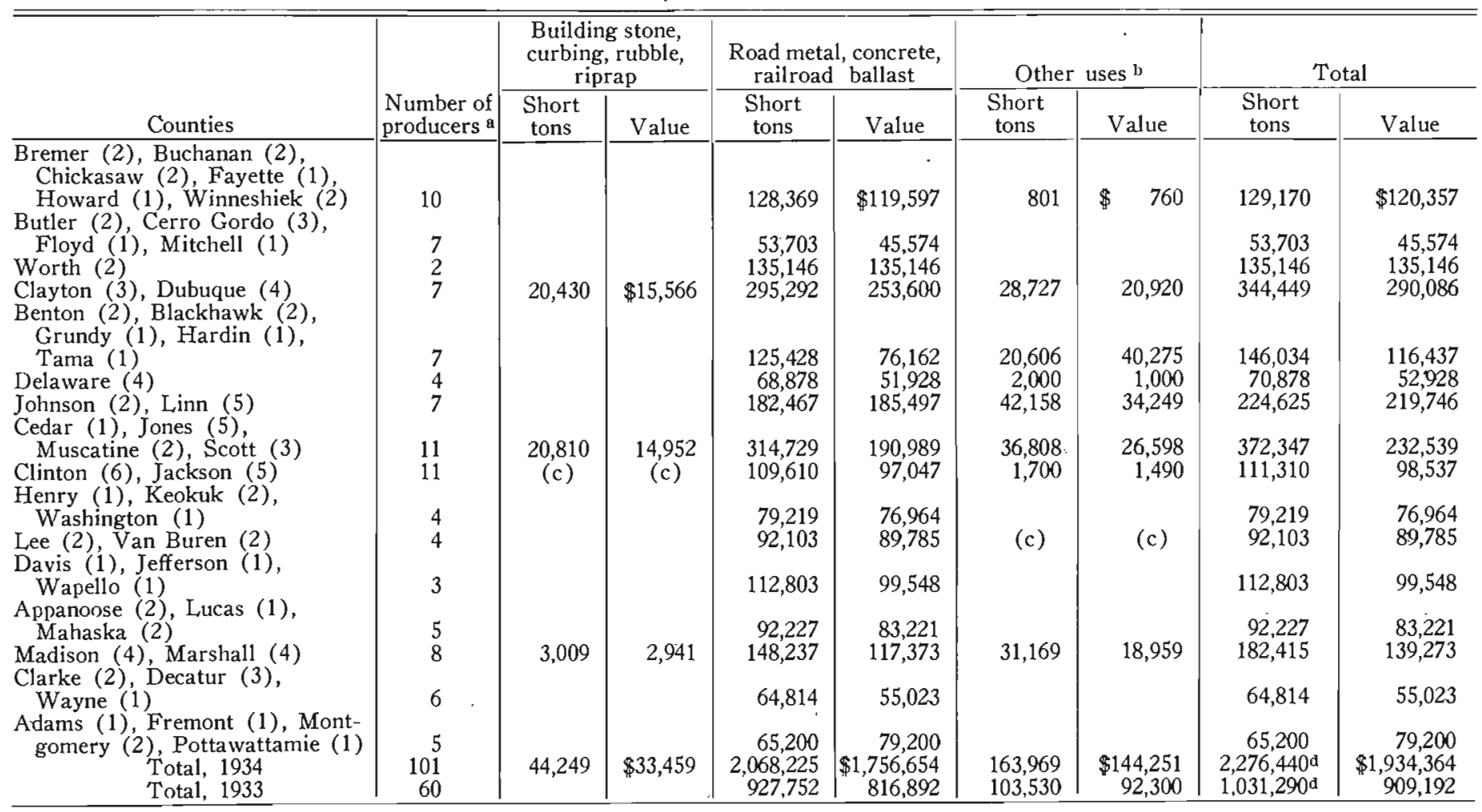

a Commercial and noncommercial

a Adjusted. 
TABLE XXVI

Production of Limestone in Iover in 1935

\begin{tabular}{|c|c|c|c|c|c|c|c|c|c|}
\hline \multirow[b]{2}{*}{ Counties } & \multirow[b]{2}{*}{$\begin{array}{l}\text { Number of } \\
\text { producers a }\end{array}$} & \multicolumn{2}{|c|}{$\begin{array}{l}\text { Building stone, } \\
\text { curbing, rubble, } \\
\text { riprap }\end{array}$} & \multicolumn{2}{|c|}{$\begin{array}{l}\text { Road metal, concrete, } \\
\text { railroad ballast }\end{array}$} & \multicolumn{2}{|c|}{ Other uses b } & \multicolumn{2}{|c|}{ Total } \\
\hline & & $\begin{array}{l}\text { Short } \\
\text { tons }\end{array}$ & Value & $\begin{array}{l}\text { Short } \\
\text { tons }\end{array}$ & Value & $\begin{array}{l}\text { Short } \\
\text { tons }\end{array}$ & Value & $\begin{array}{l}\text { Short } \\
\text { tons }\end{array}$ & Value \\
\hline $\begin{array}{l}\text { Allamakee (3), Clayton (6), } \\
\text { Dubuque (5) }\end{array}$ & 14 & 16,824 & $\$ 14,411$ & 353,371 & $\$ 284,765$ & 28,299 & $\$ 27,397$ & 398,494 & $\$ 326,573$ \\
\hline $\begin{array}{l}\text { Chickasaw (1), Fayette (3), Floyd (1), } \\
\text { Mitchell (1), Winneshiek (4) }\end{array}$ & 10 & & & 126,410 & 122,904 & & & 126,410 & 122,904 \\
\hline $\begin{array}{l}\text { Black Hawk (4), Bremer (2), } \\
\text { Buchanan (2), Delaware (3) }\end{array}$ & 11 & & & 148,827 & 134,136 & 3,846 & 3,541 & 152,673 & 137,677 \\
\hline $\begin{array}{l}\text { Cerro Gordo (1), Franklin (1), } \\
\text { Hardin (1), Marshall (3) }\end{array}$ & 6 & & & 63.557 & 56,196 & 29,835 & 57,905 & 93,392 & 114,101 \\
\hline $\begin{array}{l}\text { Benton (1), Butler (2) } \\
\text { Grundy (1), Tama (2) } \\
\text { Jackson (2), Johnson (3), }\end{array}$ & 6 & & & 54,316 & 45,297 & & & 54,316 & 45,297 \\
\hline $\begin{array}{l}\text { Jones (5), Linn (7) } \\
\text { Cedar (1), Clinton (1), Louisa (1), }\end{array}$ & 17 & 20,474 & 14,804 & 134,214 & 123,143 & 29,270 & 22,619 & 183,958 & 160,566 \\
\hline Muscatine (2), Scott (3) & 8 & 20,809 & 16,988 & 124,903 & 94,264 & 32,610 & 25,024 & 178,322 & 136,276 \\
\hline $\begin{array}{l}\text { Keokuk (2), Washington (1) } \\
\text { Jefferson (1), Mahaska (2) }\end{array}$ & 5 & & & 112,830 & 109,356 & & & 112,830 & 109,356 \\
\hline Wapello (1) Davis (i) & 4 & & & 102,786 & 100,764 & & & 102,786 & 100,764 \\
\hline $\begin{array}{l}\text { Appanoose }(1) \text {, Davis (1), } \\
\text { Lee (3), Van Buren (3) } \\
\text { Clarke (1), Decatur (2) }\end{array}$ & 8 & & & 131,870 & 121,888 & (c) & (c) & 131,870 & 121,888 \\
\hline $\begin{array}{l}\text { Madison (4), Wayne (2) } \\
\text { Adair (2), Cass (1). }\end{array}$ & 9 & (c) & (c) & 179,959 & 149,026 & 9,623 & 7,263 & 189,582 & 156,289 \\
\hline Pottawattamie (1) & 4 & & & 83,792 & 82,599 & & & 83,792 & 82,599 \\
\hline Fremont (2), Montgomery (1) & 3 & & & 31,647 & 31,647 & & & 31,647 & 31,647 \\
\hline $\begin{array}{l}\text { 1otal, } 1935 \\
\text { Total, } 1934\end{array}$ & $\begin{array}{l}105 \\
101\end{array}$ & $\begin{array}{l}58,107 \\
44,249\end{array}$ & $\begin{array}{r}\$ 46,203 \\
33,459\end{array}$ & $\begin{array}{l}1,048,482 \\
2,068,225\end{array}$ & $\begin{array}{r}\$ 1,455,985 \\
1,756,654\end{array}$ & $\begin{array}{l}133,483 \\
163,969\end{array}$ & $\begin{array}{r}\$ 143,749 \\
144,251\end{array}$ & $2,276,440^{a}$ & $\begin{array}{r}\$ 1,045,937 \\
1.934,364\end{array}$ \\
\hline
\end{tabular}

a Commercial and noncommercial.

limer factories, and other uses

c Included in road metal, concrete and railroad ballast column for purpose of concealment. 
TABLE XXVII

Production of Limestone in Iowa in 1936

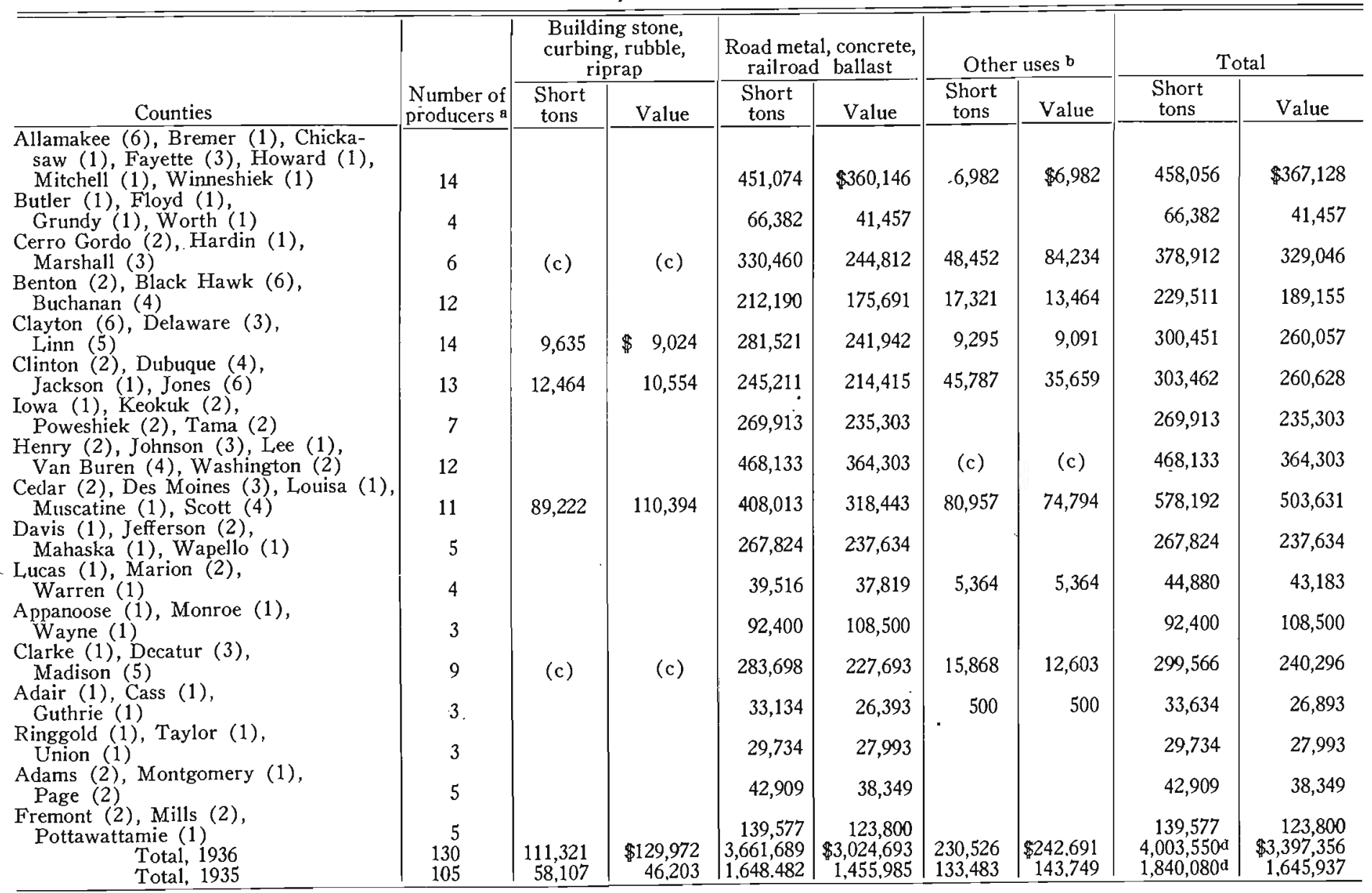


TABLE XXVIII

Production of Limestone in Iowa in 1937

\begin{tabular}{|c|c|c|c|c|c|c|c|c|c|}
\hline \multirow[b]{2}{*}{ Counties } & \multirow[b]{2}{*}{$\begin{array}{c}\text { Number of } \\
\text { producers }\end{array}$} & \multicolumn{2}{|c|}{$\begin{array}{l}\text { Building stone, } \\
\text { curbing, rubble, } \\
\text { riprap }\end{array}$} & \multicolumn{2}{|c|}{$\begin{array}{l}\text { Road metal, concrete, } \\
\text { railroad ballast }\end{array}$} & \multicolumn{2}{|c|}{ Other uses b } & \multicolumn{2}{|c|}{ Total } \\
\hline & & $\begin{array}{l}\text { Short } \\
\text { tons }\end{array}$ & Value & $\begin{array}{l}\text { Short } \\
\text { tons }\end{array}$ & Value & $\begin{array}{l}\text { Short } \\
\text { tons }\end{array}$ & Value & $\begin{array}{c}\text { Short } \\
\text { tons }\end{array}$ & Value \\
\hline $\begin{array}{l}\text { Allamakee (5), Clayton (6), } \\
\text { Dubuque (4) }\end{array}$ & & & & & & & & & \\
\hline Chickasaw (1), Howard (1), & 15 & 188,701 & $\$ 127,792$ & 295,702 & $\$ 295,906$ & 12,322 & $\$ 12,422$ & 496,725 & $\$ 436,120$ \\
\hline $\begin{array}{l}\text { Winneshiek (3) } \\
\text { Cerro Gordo (1), Hardin (3), }\end{array}$ & 5 & & & 88,206 & 102,175 & 5,072 & 3,297 & 93,278 & 105,472 \\
\hline $\begin{array}{l}\text { Mitchell (3) } \\
\text { Kossuth (1), Palo Alto (1) }\end{array}$ & $\begin{array}{l}7 \\
2\end{array}$ & $\begin{array}{r}130 \\
1,750\end{array}$ & $\begin{array}{r}15 \\
938\end{array}$ & 50,065 & 47,456 & 29,114 & 65,317 & $\begin{array}{r}79,309 \\
1,750\end{array}$ & $\begin{array}{r}112,788 \\
938\end{array}$ \\
\hline $\begin{array}{l}\text { Bremer (3), Butler (2) } \\
\text { Fayette (4). Floyd (4) }\end{array}$ & & & & & & & & & \\
\hline Black Hawk (5), Grundy (2), & 13 & & & 187,690 & 176,621 & 12,586 & 13,087 & 200,276 & 189,708 \\
\hline $\begin{array}{l}\text { Marshall (5) } \\
\text { Benton (3), Buchanan (2), }\end{array}$ & 12 & (c) & (c) & 321,667 & 325,776 & 18,159 & 14,532 & 339,826 & 340,308 \\
\hline Delaware (2), Linn (7) & 14 & 98 & 75 & 353,372 & 306,994 & 51,956 & 51,419 & 405,426 & 358,488 \\
\hline $\begin{array}{l}\text { Cedar (3), Johnson (4), Jones (6) } \\
\text { Clinton (2), Jackson (4), }\end{array}$ & 13 & 14,744 & 12,349 & 268,985 & 249,124 & 26,577 & 19,632 & 310,306 & 281,105 \\
\hline $\begin{array}{l}\text { Muscatine (5), Scott (4) } \\
\text { Des Moines (4), Henry (2), } \\
\text { Lee (2), Louisa (1), }\end{array}$ & 15 & 58,683 & 58,253 & 303,763 & 269,208 & 89,878 & 91,101 & 452,324 & 418,562 \\
\hline Van Buren (3), Washington (3) & 15 & 4,760 & 3,161 & 451,071 & 474,244 & 273 & 245 & 456,104 & 477,650 \\
\hline $\begin{array}{l}\text { Jefferson (3), Keokuk (3) } \\
\text { Mahaska (4), Powesliek (1), }\end{array}$ & 6 & 3,112 & 1,756 & 178,982 & 196,745 & 1,630 & 1,467 & 183,724 & 199,968 \\
\hline $\begin{array}{c}\text { Tama (1) } \\
\text { Appanoose (3), Davis (2) }\end{array}$ & 6 & 500 & 500 & 225,230 & 241,973 & 1,000 & 850 & 226,730 & 243,323 \\
\hline $\begin{array}{l}\text { Wapello (3) } \\
\text { Lucas (2), Monroe (2), Wayne (1) } \\
\text { Clarke (2), Decatur (4), Madison (7), }\end{array}$ & $\begin{array}{l}8 \\
5\end{array}$ & & & $\begin{array}{l}215,121 \\
184,155\end{array}$ & $\begin{array}{l}232,195 \\
188,950\end{array}$ & 991 & 754 & $\begin{array}{l}216,112 \\
184,155\end{array}$ & $\begin{array}{l}232,949 \\
188,950\end{array}$ \\
\hline $\begin{array}{l}\text { Marion (2), Warren (1) } \\
\text { Adair (2), Dallas (1), Guthrie (1) }\end{array}$ & $\begin{array}{r}16 \\
4\end{array}$ & (c) & (c) & $\begin{array}{r}283,942 \\
31,335\end{array}$ & $\begin{array}{r}254,751 \\
37,300\end{array}$ & $\begin{array}{r}86,175 \\
1,000\end{array}$ & $\begin{array}{r}96,345 \\
1,250\end{array}$ & $\begin{array}{r}370,117 \\
32,335\end{array}$ & $\begin{array}{r}351,096 \\
38,550\end{array}$ \\
\hline Adams (3), Ringgold (1), Union (2) & 6 & 420 & 225 & 104,670 & 133,183 & 360 & 300 & 105,450 & 133,708 \\
\hline $\begin{array}{l}\text { Montgomery (3), Page (1), Taylor (1) } \\
\text { Fremont (2), Mills (2) }\end{array}$ & 5 & 840 & 450 & 11,039 & 24,213 & & & 11,879 & 24,663 \\
\hline Pottawattamie (2) & 6 & & & 105,681 & 125,338 & & & 105,681 & 125,338 \\
\hline Undistributed (noncommercial) & 1 & 37340 & 0205514 & 1,150 & $\begin{array}{r}1,500 \\
\$ 2683,652\end{array}$ & & & $\begin{array}{c}1,150 \\
1,1501\end{array}$ & 1,500 \\
\hline Total, 1936 & 130 & 111,321 & $\mid \begin{array}{r}\$ 20,9072 \\
129,972\end{array}$ & $3,661,689$ & $\begin{array}{l}\$, 005,052 \\
3,024,693\end{array}$ & 230,526 & 242,691 & $4,003,550 \mathrm{~d}$ & $\begin{array}{r}\$ 4,391,354 \\
3,397,356\end{array}$ \\
\hline
\end{tabular}

a Commercial and noncommercial.

b Includes limestone for agriculture, flux, sugar factories, and other uses.
o Included in road metal, concrete and railroad ballast column for purpose of concealment.

d Adjusted. 
TABI.E XXIX

Production of Limestone in Iowa in 1938

\begin{tabular}{|c|c|c|c|c|c|c|c|c|c|}
\hline \multirow[b]{2}{*}{ Counties } & \multirow[b]{2}{*}{$\begin{array}{l}\text { Number of } \\
\text { producers a }\end{array}$} & \multicolumn{2}{|c|}{$\begin{array}{l}\text { Building stone, } \\
\text { curbing, rubble, } \\
\text { riprap }\end{array}$} & \multicolumn{2}{|c|}{$\begin{array}{l}\text { Road metal, concrete, } \\
\text { railroad ballast }\end{array}$} & \multicolumn{2}{|c|}{ Other uses b } & \multicolumn{2}{|c|}{ Total } \\
\hline & & $\begin{array}{l}\text { Short } \\
\text { tons }\end{array}$ & Value & $\begin{array}{l}\text { Short } \\
\text { tons }\end{array}$ & Value & $\begin{array}{l}\text { Short } \\
\text { tons }\end{array}$ & Value & $\begin{array}{l}\text { Short } \\
\text { tons }\end{array}$ & Value \\
\hline Allamakee (2), Clayton (4) & & & & & & & & & \\
\hline $\begin{array}{l}\text { Dubuque (2), Jackson (3) } \\
\text { Cerro Gordo (1) Floyd (2) }\end{array}$ & 11 & 75,026 & $\$ 39,516$ & 73,818 & $\$ 57,971$ & 14,328 & $\$ 13,612$ & 163,172 & $\$ 111,099$ \\
\hline Howard (2), Mitchell (1) & 6 & (c) & (c) & 31,834 & 22,152 & 18,165 & 31,537 & 49,999 & 53,689 \\
\hline $\begin{array}{l}\text { Butler (1), Fayette (2) } \\
\text { Buter }\end{array}$ & 8 & 750 & 1,000 & 91,893 & 67,812 & 13,075 & 11,465 & 105,718 & 80,277 \\
\hline Delaware (1), Jones (6) & 7 & 6,851 & 5,579 & 119,080 & 80,771 & 22,969 & 16,177 & 148,900 & 102,527 \\
\hline $\begin{array}{l}\text { Clinton (3), Muscatine (3), Scott (2) } \\
\text { Johnson (2) Linn (5) }\end{array}$ & 8 & 41,297 & 30,588 & 179,112 & 136,798 & 88,867 & 70,260 & 309,276 & 237,646 \\
\hline $\begin{array}{l}\text { Washington (1) } \\
\text { Hardin (2) Marion (1) }\end{array}$ & 8 & 4,785 & 3,742 & 151,844 & 145,842 & 13,312 & 13,012 & 169.941 & 162,596 \\
\hline Marshal1' (2), Story (1) & 6 & & & 92,920 & 82,288 & 36,396 & 86,616 & 129,316 & 168,904 \\
\hline $\begin{array}{l}\text { Des Moines (1), Lee (1), } \\
\text { Louisa (1) Van Buren (5) }\end{array}$ & 8 & (c) & (c) & 106,068 & 85,751 & 29,767 & 24,925 & 135,835 & 110,676 \\
\hline $\begin{array}{l}\text { Keokuk (1), Mahaska (1), } \\
\text { Wapello (1) }\end{array}$ & 3 & & & 77,284 & 69,044 & & & 77,284 & 69,044 \\
\hline Appanoose (1), Lucas (2), & & & & , & . & 700 & 7000 & & \\
\hline Decatur (1), Guthrie (2), Harrison (1), & 4 & 300 & 225 & 42,240 & 51,940 & , & , OWU & 47,040 & 59,105 \\
\hline $\begin{array}{l}\text { Madison (2), Ringgold (2) } \\
\text { Adanis (1) Fremont (1) }\end{array}$ & 8 & (c) & (c) & 140,255 & 94,976 & 11,891 & 10,320 & 152,146 & 105,146 \\
\hline Montgomery (1) & 3 & & & 23,250 & 20,000 & 1,715 & 2,373 & 24,965 & 22,373 \\
\hline Undistributed (noncommercial) & 5 & 54,370 & 32,750 & $1,726,343$ & $2,400,674$ & 26,945 & 25,864 & $1,807,652$ & $2,459,280$ \\
\hline Total, 1938 & 85 & 183,379 & 113,400 & $2,855,941$ & $3,316,019$ & 284,430 & $3: 13,161$ & $3,323,750$ & $3,742,580$ \\
\hline Total, 1937 & 174 & $273,734 \mathrm{~d}$ & 205,514 & $3,661.826$ & $3,683,652$ & $337,110^{d}$ & 372,018 & $4,272,670^{d}$ & $4,261,184$ \\
\hline
\end{tabular}

c Included in road metal, concrete and railroad ballast column for purpose of concealment.

d Adjusted. 


\section{Limestone Operators in Iowa}

Adair County

Noncommercial

Adair County Highway Department, County Engineer, Greenfield, Iowa.

Adams County

Adams County Highway Department, Corning, Iowa.

City of Corning, Highway Department, Corning, Iowa.

Allamakee County

Allamakee County Highway Department, Waukon, Iowa.

Appanoose County

Appanoose County Highway Department, County Engineer, Centerville, Iowa.

C. C. C. Camp 14, Drakesville, Iowa.

Benton County

Benton County Highway Department, Vinton, Iowa.

Blackhawk County

Blackhawk County Highway Department, Waterloo, Iowa.

City of Cedar Falls, Cedar Falls, Iowa.

City of Waterloo, Waterloo, Iowa.

Bremer County

Bremer County Highway Department, Waverly, Iowa.

Buchanan County

Buchanan County, County Engineer, Independence, Iowa.

Butler County

Butler County, County Engineer, Allison, Iowa.

Cass County

Cass County Highway Department, Atlantic, Iowa.

Cedar County

Cedar County Highway Department, Tipton, Iowa.

Cerro Gordo County

Cerro Gordo County Highway Department, Mason City, Iowa.

Chickasaw County

Chickasaw County, County Engineer, New Hampton, Iowa.

Clarke County

Clarke County, County Engineer, Osceola, Iowa.

Clayton County

Clayton County, County Engineer, Elkader, Iowa. 
Clinton County

Clinton County Highway Department, Clinton, Iowa.

Davis County

Davis County Highway Department, Bloomfield, Iowa.

Decatur. County

Decatur County Highway Department, Leon, Iowa.

Soil Conservation Committee, Grand River, Iowa.

Delaware County

Delaware County, County Engineer, Manchester, Iowa.

Des Moines County

City of Burlington, Burlington, Iowa.

Des Moines County, County Engineer, Burlington, Iowa.

Dubuque County

Dubuque County Highway Department, County Engineer, Dubuque, Iowa.

Fayette County

Fayette County Highway Department, West Union, Iowa.

Floyd County

Floyd County Highway Department, Charles City, Iowa.

Franklin County

Franklin County, County Engineer, Hamilton, Iowa.

Fremont County

Fremont County, County Engineer, Sidney, Iowa.

Fremont County Soil Association, Farragut, Iowa.

Grundy County

Grundy County Highway Department, Grundy Center, Iowa.

Guthrie County

County Agricultural Agent, Guthrie, Iowa.

Henry County

Henry County, County Engineer, Mt. Pleasant, Iowa.

Supervisor W. P. A., Mt. Pleasant, Iowa.

Howard County

Howard County, County Engineer, Cresco, Iowa.

Humboldt County

City of Humboldt Highway Department, Humboldt, Iowa.

Jackson County

Jackson County Highway Department; Maquoketa, Iowa. 
Jefferson County.

Jefferson County Highway Department, County Engineer, Fairfield, Iowa.

Johnson County

Johnson County Highway Department, County Engineer, Iowa City, Iowa.

Jones County

Jones County, County Engineer, Anamosa, Iowa.

Men's Reformatory, Anamosa, Iowa.

Keokuk County

Keokuk County, County Engineer, Sigourney, Iowa.

Lee County

Lee County Highway Department, Fort Madison, Iowa.

Linn County

City of Cedar Rapids Street Department, Cedar Rapids, Iowa.

Linn County, County Engineer, Cedar Rapids, Iowa.

Louisa County

Louisa County, County Engineer, Wapello, Iowa.

Lucas County

Lucas County, County Engineer, Chariton, Iowa.

I,ucas County Farm Bureau, County Board of Supervisors, Chariton, Iowa.

Madison County

Madison County Highway Department, Winterset, Iowa.

Mahaska County

Mahaska County, County Engineer, Oskaloosa, Iowa.

Marion County

Soil Conservation Service, Knoxville, Iowa.

Marshall County

Marshall County, County Engineer, Marshalltown, Iowa.

Mills County

Mills County, County Engineer, Glenwood, Iowa.

Mitchell County

Mitchell County, County Highway Engineer, Osage, Iowa.

Monroe County

Monroe County, County Engineer, Albia, Iowa.

Montgomery County

Montgomery County Highway Department; Red Oak, Iowa. 
U. S. Department of Agriculture, Soil Conservation Association, C. C. C., Red Oak, Iowa.

Muscatine County

Muscatine County Highway Department, Muscatine, Iowa.

Works Progress Administration, Supervisor, Conesville, Iowa. Page County

Soil Conservation Service, Experiment Station, Box 341, Clarinda, Iowa.

Pottaz'attamic County

Pottawattamie County Highway Department, Council Bluffs, Iowa.

Poweshiek County

Poweshiek County Highway Department, Montezuma, Iowa.

Ringgold County

Ringgold County, County Enginer, Mt. Ayr, Iowa.

Scott County

Scott County Highway Department, Davenport, Iowa.

Tama County

Tama County Highway Department, Toledo, Iowa.

Taylor County

Taylor County Highway Department, Bedford, Iowa.

Union County

Union County, Board of County Supervisors, Creston, Iowa.

Van Buren County

Farm Bureau Soils Committee, Keosauqua, Iowa.

Van Buren County Highway Department, Keosauqua, Iowa.

Wapello County

Wapello County Highway Department, Ottumwa, Iowa.

Warren County

Warren County Highway Department, Indianola, Iowa.

Washington County

Washington County Highway Department, Washington, Iowạ.

Wayne County

Wayne County Highway Department, Corydon, Iowa.

Winneshiek County

Winneshiek County Highway Department, Decorah, Iowa.

Worth County

Worth County Highway Department, Northwood, Iowa.

State of Iowa

Property \& Epuipment Division, Ames, Iowa. 
Administrator, Iowa Works Progress Administration, Royal Union - Life Building, Des Moines, Iowa.

\section{Limestone Operators in Iowa \\ Commercial}

Allamakee County

Halvorson Bros., Rochester, Minnesota. Quarry at Lansing.

Roverud Bros., Spring Grove, Minnesota. Pool Hill Quarry at

New Albin.

E. C. Schroeder, McGregor, Iowa. Martin Manton Quarry at Harpers Ferry.

Hess Bros., Lansing, Iowa. Johnson Quarry.

Edward Anderson, Lansing, Iowa.

Appanoose County

Centerville Limestone Co., Centerville, Iowa.

Dixon Construction Co., Centerville, Iowa.

Benton County

Say Raymond, Garrison, Iowa.

Black Hawk County

A. C. Newton, LaPorte, Iowa.

Concrete Materials Co., Box 790, Cedar Rapids, Iowa. Portable Plants.

Gill \& Mullen, LaPorte City, Iowa.

R. G. Holm, Waterloo, Iowa.

Harold E. Pint, Raymond, Iowa.

Frank Frost, Jesup, Iowa.

Bremer County

Joseph Alcock, New Hampton, Iowa. Fredrika Quarry, Fredrika, Iowa.

Schield Bros., Waverly, Iowa. Colburn Quarry.

Buchanan County

Lewis V. T. Francis, Fairbank, Iowa. Quarry at Jesup.

Butler County

R. W. Phillips, Rockwell, Iowa. Portables.

Carroll County

Emery Construction Co., Coon Rapids, Iowa.

Cedar County

Donald P. Thomson, Mt. Vernon, Íowa. Quarry at Mechanicsville. 
Cerro Gordo County

N. W. States Portland Cement Co., Mason City, Iowa.

Stoddard Stone Products Co., 500 14th Street, N. E., Mason City, Iowa.

Clarke County

W. C. Busick, Osceola, Iowa.

Willis Busick, Osceola, Iowa.

Clayton County

Eberhard Construction Co., Guttenberg, Iowa.

H. L. Leas, Monona, Iowa.

E. C. Schroeder, McGregor, Iowa.

Clinton County

Harry Belby, Charlotte, Iowa.

C. T. Hanrahan, Charlotte, Iowa.

Adolf Thiessen, Charlotte, Iowa.

George T. Smith, 715 Sixth Avenue, South, Clinton, Iowa. Nagel's

Quarry at Lyons.

Decatur County

Sargent Bros., Inc., 411 E. Grand Avenue, Des Moines, Iowa. Quarry at Decatur.

Des Moines County

Henry Roscum, Burlington, Iowa.

Dubuque County

Dubuque Stone Products Co., 2900 Rhomberg Avenue, Dubuque, Iowa.

John Rider Wallis, Dubuque, Iowa. Horseshoe Bluff Quarries.

M. F. Simon, Farley, Iowa.

Fayette County.

L. H. Stranahan, Fayette, Iowa.

Floyd County

Clyde Stevens, Floyd, Iowa. Knowlton Quarry.

E. J. Wilcox and Sons, Floyd, Iowa.

Fremont County

Hartsell \& Evans, Thurman, Iowa.

Guthrie County

Boyd Crandall, Guthrie Center, Iowa.

Fred B. Owen, Guthrie Center, Iowa. Quarry at Redfield.

Hardin County

Pearce Limestone Corp., Gifford, Iowa. 
Iowa Limestone Co., 907 Bankers Trust Building, Des Moines, Iowa. Quarry at Alden.

M. B. Musgrave, Woodbine, Iowa.

Henry County

Hannill Limestone Co., Lockridge, Iowa.

Paul Niemann, West Union, Iowa.

Iowa County

E. D. Wahl, Victor, Iowa.

Jackson County

C. C. Putnam, Hager City, Wisconsin. Riching \& Keeney Quarry.

H. C. Roberts, Maquoketa, Iowa.

Hurst Stone Co., R. R. No. 3, Maquoketa, Iowa.

Johnson County

River Products Co., 20-21 Schneider Building, Iowa City, Iowa. Quarry at Coralville.

Jones County

Myron Baker Contractor, Independence, Iowa. Portable crúshers all over Iowa.

Charles W. Zimmer, Anamosa, Iowa. Quarry in Cass township. Columbia Quarry, G. J. Albright, 612 C Avenue, N. W., Cedar Rapids, Iowa. Columbia Quarry, Stone City.

Merle Ballou, Olin, Iowa.

Willis Johnson, Stone City, Iowa.

Fall Brothers, Olin, Iowa.

H. Dearborn Sons, Stone City, Iowa. Stone City Quarry.

Malcolm Vernon, Olin, Iowa.

Theodore Patnode, Stone City, Iowa.

Lee County

McManus Quarries Co., Inc., 112 Masonic Building, Keokuk, Iowa.

Driscoll \& Hayes, Farmington, Iowa. Quarry at Belfast.

Fred Osborne, Denmàrk, Iowa.

Keokuk Quarry \& Construction Co., 1325 Main Street, Keokuk, Iowa.

Oral France, Martinsburg, Iowa. Quarry at Ollie, Iowa.

\section{Linn County}

Larimer \& Shafer, Inc., E. Moore, Recorder, First Avenue and Second Street, Cedar Rapids, Iowa.

Art Lanning, Alburnett, Iowa. Lafayette Quarry.

J. G. Vernon, Marion, Iowa. 
Lanning \& Fulkerson, Marion, Iowa.

G. W. Gaines, Lisbon, Iowa.

Dewees \& Whitney, Marion, Iowa.

Dewees \& Smith, R. F. D. No. 1, Springville, Iowa.

I. H. Whitman, Lisbon, Iowa.

John Vernon, Springville, Iowa.

Dan Thompson, Mt. Vernon, Iowa.

A. R. Gaines, Mt. Vernon, Iowa.

\section{Madison County}

$\therefore$ Hawkeye Portland Cement Co., 802 Hubbell Building, Des Moines, Iowa. Quarry at Earlham.

Sargent Bros., Inc., 411 E. Grand Avenue, Des Moines, Iowa. Quarry at Winterset.

Winterset Limestone Co, Winterset, Iowa.

Madison County Limestone Co., Winterset, Iowa.

Mahaska County

John P. Abramson Construction Co., Des Moines, Iowa.

Marion County

Pella Limestone Co., Knoxville, Iowa. Quarry at Pella.

A. K. Verrifs, Pella, Iowa.

E. Groenendyke, Tracy, Iowa.

\section{Marshall County}

Chicago \& N. W. Ry. Co., Quarry at Marshalltown, Iowa.

LeGrand Limestone Co., 105 W. Madison Street, Chicago, Illinois. Quarry at Lake View, Iowa.

\section{Mitchell County}

Falk \& Litzelman, Osage, Iowa. Quarry at Rudolph Nitardy at St. Ansgar. Quarry of Gaylord Snyder at Osage, Iowa.

Kollman-Bros., Osage, Iowa. Quarry at New Haven.

H. L. Wilson Estate, Osage, Iowa. Osage Stone Quarry.

\section{Montgomery County}

Albert Mulvenna, Red Oak, Iowa.

\section{Muscatine County}

C. C. Putnam, Hager City, Wisconsin. Schroder Quarry at Montpelier or Princeton.

Otto Wendling, 1549 Washington Street, Muscatine, Iowa. Quarry at Moscow. 


\section{Pocahontas County}

N. W. States Portland Cement Co., Gilmore Portland Cement Corp., Mason City, Iowa.

Pottawattamie County

Kelley Construction Co., 532 46th Street, Des Moines, Iowa. Quarry at Macedonia.

Ringgold County

Harco Construction Co., Mount Ayr, Iowa. Watterson Quarry. Scott County

Dewey Portland Cement Co., 409 Scarrett Building; Kansas City, Missouri.

Falk \& Litzelman, Osage, Iowa. Quarry at"Bettendorf.

Linwood Stone Products Co., 928 Davenport Bank Building., Davenport, Iowa. Quarry at Linwood.

Story County

Maudlin Construction Co., Box 134, Webster City, Iowa.

Nelson \& Malone, Nevada, Iowa.

Ray Cook, Nevada, Iowa. Quarry at Ames.

Tama County

B. L. Anderson, Toledo, Iowa.

Lake Park Holding Corp., Gladbrook, İowa. :

Van Buren County

Roberts Willits, Bonaparte, Iowa. Mud Creek Quarry.

Douds Quarries, Inc., Douds, Iowa.

Washington County

J. C. Smay, Nevada, Iowa. Grace Hill Quarry.

Webster County

Fort Dodge Lime Stone Co., Fort Dodge, Iowa.

Winneshiek County

Decorah Stone Products Co., Decorah, Iowa.

Cremer Construction Co., Decorah, Iowa.

T. D. Jeglum, Decorah, Iowa.

\section{Sand and Gravel}

Principal uses of sand and gravel in Iowa are for paving and roads, and for structural purposes. The chief secondary use is for railroad ballast. Normally the total value of gravel is approximately four times that of sand. Table XXX summarizes the outstanding features of this industry in Iowa from 1933 to 1938 , and reports by counties may 
TABLE XXX

Summary of Sand and Gravel Production in Iowa from 1932 to 1938

\begin{tabular}{|c|c|c|c|c|c|c|c|c|c|c|c|c|c|c|c|}
\hline & 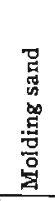 & 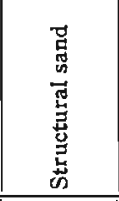 & 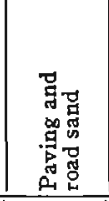 & 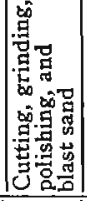 & 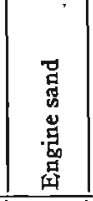 & 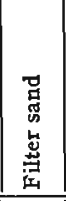 & 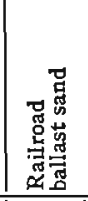 & 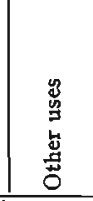 & 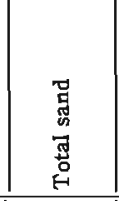 & 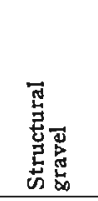 & 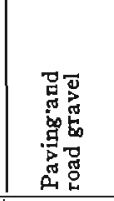 & 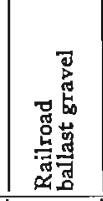 & 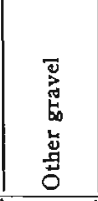 & 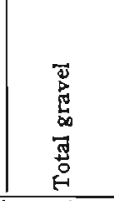 & 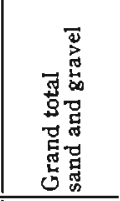 \\
\hline $\begin{array}{l}1932 \\
\text { Short tons } \\
\text { Value } \\
1933\end{array}$ & (a) & $\begin{array}{r}288,719 \\
\$ 118,866\end{array}$ & $\begin{array}{l}827,883 \\
204,192\end{array}$ & $\begin{array}{l}6,111 \\
8,315\end{array}$ & $\mid \begin{array}{l}22,277 \\
10,449\end{array}$ & (a) & $\left|\begin{array}{l}45,054 \\
14,933\end{array}\right|$ & $\begin{array}{l}14,124 \\
11,896\end{array}$ & $\begin{array}{r}1,204,168 \\
368,651\end{array}$ & $\begin{array}{l}289,349 \\
219,651\end{array}$ & $\begin{array}{l}3,422,195 \\
1,063,008\end{array}$ & $\begin{array}{r}308,059 \\
52,806\end{array}$ & $\begin{array}{l}6,791 \\
2,758\end{array}$ & $\begin{array}{l}14,026,394 \\
1,338,223\end{array}$ & $\begin{array}{l}15,230,562 \\
1,706,874\end{array}$ \\
\hline $\begin{array}{l}\text { Short tons } \\
\text { Value } \\
1934\end{array}$ & (a) & $\begin{array}{r}228,170 \mathrm{~b} \\
\$ 115,182 \mathrm{~b}\end{array}$ & $\begin{array}{l}420,607 \mathrm{~b} \\
117,451 \mathrm{~b}\end{array}$ & $\begin{array}{l}\text { (a) } \\
\text { (a) }\end{array}$ & \begin{tabular}{|}
26,896 \\
12,735
\end{tabular} \mid & $\begin{array}{l}1,336 \\
4,302\end{array}$ & (a) & $\begin{array}{l}59,703 \\
32,278\end{array}$ & $\begin{array}{l}136,712 \\
281,948\end{array}$ & $\begin{array}{l}156,520 \\
126,591\end{array}$ & $\begin{array}{r}3,2,33,422 \\
703,859\end{array}$ & $\begin{array}{r}208,842 \\
45,260\end{array}$ & $\begin{array}{l}8,285 \\
7,408\end{array}$ & $\begin{array}{r}3,607,069 \\
883,118\end{array}$ & $\begin{array}{l}4,343,781 \\
1,165,066\end{array}$ \\
\hline $\begin{array}{l}\text { Short tons } \\
\text { Value } \\
1935\end{array}$ & $\begin{array}{l}\text { (a) } \\
\text { (a) }\end{array}$ & $\begin{array}{r}369,720 \\
\$ 169,441\end{array}$ & $\begin{array}{l}459,031 \\
151,145\end{array}$ & (a) & $\begin{array}{r}25,143 \\
9,716\end{array}$ & $\begin{array}{l}1,951 \\
6,122\end{array}$ & $\begin{array}{r}17,318 \\
3,944\end{array}$ & $\begin{array}{l}48,795 \\
35,516\end{array}$ & $\begin{array}{l}921,958 \\
375,884\end{array}$ & $\begin{array}{l}266,272 \\
216,733\end{array}$ & $\begin{array}{r}3,011,978 \\
776,670\end{array}$ & $\begin{array}{l}\text { (c) } \\
\text { (c) }\end{array}$ & $\begin{array}{r}148,654 \\
25,513\end{array}$ & $\begin{array}{l}3,426,904 \\
1,017,916\end{array}$ & $\begin{array}{l}4,349,362 \\
1,394,000\end{array}$ \\
\hline $\begin{array}{l}\text { Short tons } \\
\text { Value } \\
1936\end{array}$ & (a) & $\begin{array}{r}368,416 \\
\$ 176,530\end{array}$ & $\begin{array}{l}463,189 \\
132,652\end{array}$ & $\begin{array}{l}\text { (a) } \\
\text { (a) }\end{array}$ & $\left|\begin{array}{|l|}27,234 \\
11,425\end{array}\right|$ & $\begin{array}{l}\text { (a) } \\
\text { (a) }\end{array}$ & $\left|\begin{array}{r}15,249 \\
4,538\end{array}\right|$ & $\begin{array}{r}116,375 \\
88,518\end{array}$ & $\begin{array}{l}990,463 \\
413,663\end{array}$ & $\begin{array}{l}278,041 \\
223,840\end{array}$ & $\begin{array}{l}4,238,618 \\
1,041,962\end{array}$ & $\begin{array}{l}\text { (c) } \\
\text { (c) }\end{array}$ & $\left(\begin{array}{l}c \\
c\end{array}\right)$ & $\begin{array}{l}4,742,270 \\
1,343,188\end{array}$ & $\begin{array}{l}5,732,742 \\
1,756,851\end{array}$ \\
\hline $\begin{array}{l}\text { Short tons } \\
\text { Value } \\
1937\end{array}$ & $\begin{array}{l}\text { (a) } \\
\text { (a) }\end{array}$ & $\begin{array}{r}545,410 \\
\$ 263,076\end{array}$ & \begin{tabular}{|l|}
511,852 \\
207,704
\end{tabular} & $\begin{array}{l}\text { (a) } \\
\text { (a) }\end{array}$ & \begin{tabular}{|}
25,956 \\
11,287
\end{tabular} & $\begin{array}{l}\text { (a) } \\
\text { (a) }\end{array}$ & $\begin{array}{l}4,705 \\
2,506\end{array}$ & $\begin{array}{l}82,428 \\
67,141\end{array}$ & $\left|\begin{array}{r}1,170,351 \\
.551,714\end{array}\right|$ & $\begin{array}{l}423,669 \\
337,561\end{array}$ & $\mid \begin{array}{l}4,258,146 \\
1,086,483\end{array}$ & $\begin{array}{r}436,639 \\
56,531\end{array}$ & $\begin{array}{r}5,179 \\
15,993\end{array}$ & $\begin{array}{l}5,123,633 \\
1,496,568\end{array}$ & $\begin{array}{l}6,293,984 \\
2,048,282\end{array}$ \\
\hline $\begin{array}{l}\text { Short tons } \\
\text { Value } \\
1938\end{array}$ & $\begin{array}{l}\text { (a) } \\
\text { (a) }\end{array}$ & $\begin{array}{r}562,244 \\
\$ 324,733\end{array}$ & $\begin{array}{l}629,155 \\
266,745\end{array}$ & (a) & \begin{tabular}{|}
40,232 \\
19,815
\end{tabular} & $\begin{array}{l}\text { (a) } \\
\text { (a) }\end{array}$ & $\begin{array}{l}\text { (a) } \\
\text { (a) }\end{array}$ & $\begin{array}{r}110,173 \\
91,502\end{array}$ & $\left|\begin{array}{r}1,341,804 \\
702,795\end{array}\right|$ & $\begin{array}{l}570,874 \\
414,162\end{array}$ & $\left|\begin{array}{l}4,347,826 \\
1,091,044\end{array}\right|$ & (d) & $\begin{array}{r}136,650 \\
27,102\end{array}$ & $\begin{array}{l}5,055,350 \\
1,532,308\end{array}$ & $\begin{array}{l}6,397,154 \\
2,235,103\end{array}$ \\
\hline $\begin{array}{l}\text { Short tons } \\
\text { Value }\end{array}$ & (a) & $\begin{array}{r}415,613 \\
\$ 236,557 \\
\end{array}$ & $\left|\begin{array}{l}623,569 \\
279,629\end{array}\right|$ & $\begin{array}{r}10,095 \\
9,759\end{array}$ & $\left|\begin{array}{l}37,885 \\
20,282\end{array}\right|$ & $\begin{array}{l}\text { (a) } \\
\text { (a) }\end{array}$ & $\left|\begin{array}{|l}18,594 \\
11,308\end{array}\right|$ & \begin{tabular}{|l|}
45,476 \\
41,452 \\
\end{tabular} & $\begin{array}{r}1,151,232 \\
598,987\end{array}$ & $\begin{array}{l}812,465 \\
356,426 \\
\end{array}$ & $\begin{array}{l}4,818,859 \\
1,275,485\end{array}$ & $\begin{array}{r}152,600 \\
31,062\end{array}$ & $\begin{array}{l}59,090 \\
37,722\end{array}$ & $\mid \begin{array}{l}5,373,761 \\
1,637,684\end{array}$ & $\begin{array}{l}6,994,286 \mathrm{e} \\
2,299,732\end{array}$ \\
\hline
\end{tabular}


be found in Tables XXXII to XLIFI. In the latter tables it was found necessary to combine counties to avoid revealing confidential data.

Total quantity and total value of all sand and gravel produced in the state, after a decrease in 1933, increased in each of the ensuing years including 1938. The greatest advance in value came in 1935 when the total was $\$ 363,051$ greater than in 1934 while the largest increase in quantity was attained in 1936 when the figures for 1935 were exceeded by $1,383,880$ tons.

The 1938 figures of $6,994,286$ ton valued at $\$ 2,299,682$ represent the greatest quantity and the highest total annual value of production in the history of the sand and gravel industry in Iowa.

Paving and road gravel after a decrease in 1933 advanced in total value each year including 1938 and sand for the same use had a similar history except for a decrease in value in 1935. Sand and gravel used for structural purposes decreased in 1933 in tonnage and total value, but increased each year thereafter until 1937, and in 1938 gravel production for this use broke all previous records.

In the United States the grand total of sand and gravel production and value dropped in.1933, increased each year from 1934 to 1937 inclusive and decreased slightly in 1938 as shown by Table XXXI.

The 1933 decreases of commercial sand and gravel amounted to 22 per cent in quantity and 17 per cent in value from 1932 . Commercial increases in 1934 amounted to 13.9 per cent in. quantity and 6.7 per cent in value while the increases in 1935 were of somewhat smaller magnitude. The greatest increase in production was attained in 1936 when there were apparent advances of 39 per cent in tonnage and 43 per cent in value. The factors responsible for this upward trend were greater activity in building and highway construction and the resulting demand for commercial sand and gravel. Increases of 6 per cent in quantity and 8 per cent in value were effective in 1937 while in 1938 commercial production dropped 16 per cent and total quantity of sand and gravel "sold or used" dropped 4 per cent.

An important feature of the sand gravel industry in 1938 was, the further tendency toward increasing use of noncommercial operations for supplies of aggregates. It is reported that the domestic output of plants operated by states, counties, municipalities and other Government agencies was 17 per cent higher in 1938 than in 1937 and represented 42 per cent of the total tonnage produced. 
TABLE XXXI

Sand and Gravel Industry in the United States from 1932 to 1938

\begin{tabular}{|c|c|c|c|c|c|c|c|c|c|c|c|c|c|c|c|c|c|}
\hline & 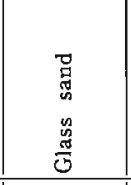 & 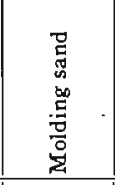 & 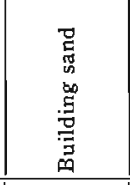 & 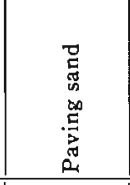 & 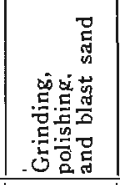 & 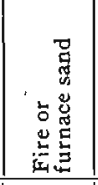 & 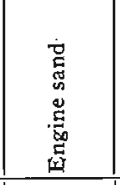 & 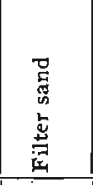 & 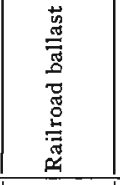 & 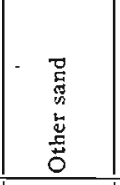 & 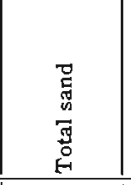 & 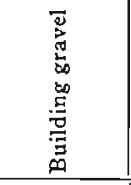 & 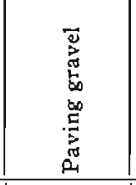 & 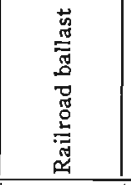 & 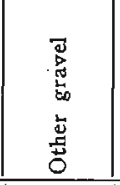 & 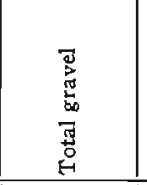 & 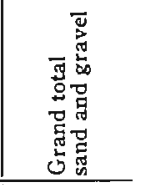 \\
\hline $\begin{array}{l}1932 \\
\text { Short tons } \\
\text { Value }\end{array}$ & $\begin{array}{l}1,370,255 \\
2,266,564\end{array}$ & $\mid \begin{array}{l}1,118,146 \\
1,051,702\end{array}$ & $\begin{array}{r}14,745,267 \\
7,604,983\end{array}$ & $\begin{array}{r}19,399,117 \\
8,635,934\end{array}$ & $\begin{array}{l}419,691 \\
638,556\end{array}$ & $\begin{array}{l}36,698 \\
54,371\end{array}$ & $\left|\begin{array}{r}1,151,011 \\
688,563\end{array}\right|$ & $\begin{array}{r}68,035 \\
92,751\end{array}$ & & & $\left|\begin{array}{l}42,794,875 \\
22,497,074\end{array}\right|$ & $\begin{array}{r}14,065,070 \\
9,803,629\end{array}$ & $\mid \begin{array}{l}56,533,460 \\
23,397,380\end{array}$ & & & $\begin{array}{l}77,243,022 \\
35,025,002\end{array}$ & \\
\hline $\begin{array}{l}\text { Short tons } \\
\text { Value } \\
1934\end{array}$ & $\begin{array}{r}1,781,423 \\
\$ 3,011,023\end{array} \mid$ & $\begin{array}{l}1,718,251 \\
1,558,738\end{array}$ & $\begin{array}{r}13,187,431 \\
6,580,311\end{array}$ & $\begin{array}{r}12,876,139 \\
6,295,569\end{array}$ & $\begin{array}{l}572,735 \\
739,222\end{array}$ & $\left|\begin{array}{l}106,133 \\
121,149\end{array}\right|$ & $\left|\begin{array}{r}1,051,695 \\
623,285\end{array}\right|$ & \begin{tabular}{|l|}
24,387 \\
52,186
\end{tabular} & & $\left|\begin{array}{r}1,842,652 \\
695,189\end{array}\right|$ & & $\begin{array}{r}12,584,953 \\
8,338,524\end{array}$ & $\begin{array}{l}56,581,914 \\
22,992,172\end{array}$ & & & & \\
\hline $\begin{array}{l}\text { Short tons } \\
\text { Value } \\
1935\end{array}$ & $\begin{array}{r}1,923,614 \\
\$ 3,326,538\end{array}$ & $\begin{array}{l}2,167,731 \\
2,169,254\end{array}$ & $\begin{array}{r}14,869,511 \\
8,342,007\end{array}$ & $\begin{array}{r}15,917,663 \\
8,165,589\end{array}$ & $\begin{array}{r}571,191 \\
1,039,614\end{array}$ & $\left|\begin{array}{l}137,000 \\
169,424\end{array}\right|$ & $\begin{array}{r}1,211,033 \\
795,648\end{array}$ & \begin{tabular}{|}
35,750 \\
85,567
\end{tabular} \mid & $\begin{array}{l}607,380 \\
166,918\end{array}$ & $\begin{array}{l}959,217 \\
620,512\end{array}$ & & & & & & & \\
\hline $\begin{array}{l}\text { Short tons } \\
\text { Value } \\
1936\end{array}$ & $\left|\begin{array}{r}2,125,761 \\
\$ 3,735,343\end{array}\right|$ & $\begin{array}{l}2,980,879 \\
2,915,173\end{array}$ & $\begin{array}{r}16,540,324 \\
8,819,712\end{array}$ & $\begin{array}{r}13,484,723 \\
6,900,996\end{array}$ & $\begin{array}{r}816,540 \\
1 ; 198,653\end{array}$ & $\left|\begin{array}{l}172,847 \\
204,477\end{array}\right|$ & $\begin{array}{r}1,389,877 \\
881,910\end{array}$ & $\begin{array}{l}49,301 \\
93,470\end{array}$ & $\begin{array}{l}997,499 \\
256,922\end{array}$ & $\left|\begin{array}{r}1,875,808 \\
860,557\end{array}\right|$ & & & & & & & \\
\hline $\begin{array}{l}\text { Short tons } \\
\text { Value } \\
1937\end{array}$ & $\begin{array}{r}2,394,710 \\
\$ 4,050,749\end{array}$ & $\begin{array}{l}4,210,017 \\
4,072,387\end{array}$ & $\begin{array}{l}28,533,156 \\
15,378,912\end{array}$ & $\begin{array}{r}20,025,606 \\
8,684,096\end{array}$ & $\begin{array}{r}934,059 \\
1,306,871\end{array}$ & $\left|\begin{array}{l}183,667 \\
201,099\end{array}\right|$ & $\begin{array}{r}1,576,432 \\
990,816\end{array}$ & $\begin{array}{r}126,248 \\
72,381\end{array}$ & $\begin{array}{r}300,102 \\
1,177,843\end{array}$ & $\left|\begin{array}{r}815,714 \\
1,195,523\end{array}\right|$ & & & & $\begin{array}{r}3,169,961 \\
11,723,535\end{array}$ & $\begin{array}{l}411,258 \\
738,423\end{array}$ & & $\begin{array}{r}90,307,752 \\
178,329,814\end{array}$ \\
\hline $\begin{array}{l}\text { Short tons } \\
\text { Value } \\
1938\end{array}$ & $\begin{array}{r}2,799,230 \\
\$ 4,746,629\end{array}$ & $\begin{array}{l}4,953,873 \\
5,239,435\end{array}$ & $\begin{array}{l}27,590,739 \\
15,405,031\end{array}$ & $\begin{array}{l}22,099,777 \\
10,644,979\end{array}$ & $\begin{array}{l}1,067,178 \\
1,440,736\end{array}$ & 258,287 & $\left|\begin{array}{l}1,802,869 \\
1,092,171\end{array}\right|$ & $\begin{array}{r}99.383 \\
182,414\end{array} \mid$ & $\left|\begin{array}{r}1,418,316 \\
334,585\end{array}\right|$ & $\left|\begin{array}{l}1,295,419 \\
1,058,162\end{array}\right|$ & $\left|\begin{array}{l}63,385,071 \\
40,412,497\end{array}\right|$ & & $\mid \begin{array}{l}85.267 .855 \\
33,201,326\end{array}$ & $\begin{array}{r}12,318,575 \\
3,757,068\end{array}$ & $\begin{array}{l}850,605 \\
575,893\end{array}$ & $\left|\begin{array}{r}126,275,352 \\
57,060,500\end{array}\right|$ & $\begin{array}{r}189,660,423 \\
97,472,997\end{array}$ \\
\hline $\begin{array}{l}\text { Short tons } \\
\text { Value }\end{array}$ & $\mid \begin{array}{r}2,109,462 \\
\$ 3,601,734\end{array}$ & $\begin{array}{r}2,319,902 \\
\mid 2,651,779 \\
\end{array}$ & $\mid \begin{array}{l}25,097,184 \\
13,779,047\end{array}$ & $\begin{array}{r}23,378,707 \\
\mid 10,762,421\end{array}$ & $\begin{array}{r}502,328 \\
754,805 \\
\end{array}$ & \begin{tabular}{|}
108,093 \\
$|124,343|$
\end{tabular} & \begin{tabular}{|}
$1,378,450$ \\
$\mid \begin{array}{r}\mid \\
\mid\end{array} 86,639$
\end{tabular} & $\begin{array}{r}93,711 \\
137,283 \\
\end{array}$ & $\begin{array}{l}786,435 \\
212,935 \\
\end{array}$ & $\begin{array}{l}1,399,556 \\
1,124,739\end{array} \mid$ & $\left|\begin{array}{l}57,1 \\
33,93,828 \\
33,93,725\end{array}\right|$ & $\begin{array}{l}26,314,759 \\
15,737,827 \\
\end{array}$ & \begin{tabular}{|}
$88,660,248$ \\
$33,579,665$
\end{tabular} & $\begin{array}{l}8,194,244 \\
2,255,355\end{array}$ & $\begin{array}{r}1,037,154 \\
414,275 \\
\end{array}$ & $\begin{array}{r}124,206,405 \\
51,987,122 \\
\end{array}$ & $\begin{array}{r}181,320,233 \\
85,922,847 \\
\end{array}$ \\
\hline
\end{tabular}


TABLE XXXXII

Total Production of Sand and Gravel in Iowa in 1933 - Sand

\begin{tabular}{|c|c|c|c|c|c|c|c|c|c|}
\hline \multirow[b]{2}{*}{ Counties } & \multirow[b]{2}{*}{ Producers } & \multicolumn{2}{|c|}{ Structural sand } & \multicolumn{2}{|c|}{ Paving sand } & \multicolumn{2}{|c|}{ Other sand $\bar{a}$} & \multicolumn{2}{|c|}{ Total sand } \\
\hline & & Tons & Value & Tons & Value & Tons & Value & Tons & Value \\
\hline $\begin{array}{l}\text { Appanoose (1), Mahaska (1), Wapello (1) } \\
\text { Black Hawk (4), Clayton (2) } \\
\text { Boone (1), Emmet (1), Humboldt (1), }\end{array}$ & $\begin{array}{l}3 \\
6\end{array}$ & $\begin{array}{r}24,756 \\
9,910\end{array}$ & $\begin{array}{r}13,310 \\
7,305\end{array}$ & $\begin{array}{l}22,849 \\
14,352\end{array}$ & $\begin{array}{l}\$, 085 \\
4,147\end{array}$ & $\begin{array}{l}1,886 \\
\text { (b) }\end{array}$ & $\$ \begin{array}{l}581 \\
(\mathrm{~b})\end{array}$ & $\begin{array}{l}49,491 \\
24,262\end{array}$ & $\begin{array}{r}\$ 21,976 \\
11,452\end{array}$ \\
\hline $\begin{array}{l}\text { Webster (3) } \\
\text { Harrison (1),Plymouth (1), Sioux (3) }\end{array}$ & $\frac{6}{5}$ & $\begin{array}{l}16,163 \\
42,013\end{array}$ & $\begin{array}{r}6,870 \\
16726\end{array}$ & $\begin{array}{l}60,910 \\
46,695\end{array}$ & 22,824 & (c) & (c) & $\begin{array}{l}77,073 \\
88708\end{array}$ & $\begin{array}{l}29,694 \\
27230\end{array}$ \\
\hline Butler (3), Cerro Gordo (2) & 5 & 23,223 & 11,271 & 66,560 & 20,92 & 3,500 & 1,725 & 93,283 & 33,92 \\
\hline Cherokee (2), Crawford (1), Sac (3) & 6 & (d) & (d) & 17,727 & 7,930 & 43,755 & 14,660 & 61,482 & 22,590 \\
\hline $\begin{array}{l}\text { lay (2), Lyon (1) } \\
\text { Clinton (2), Dubuque (2), Jackson (1) }\end{array}$ & $\begin{array}{l}3 \\
5\end{array}$ & $\begin{array}{c}\text { (b) } \\
14,578\end{array}$ & $\begin{array}{l}\text { (b) } \\
6,392\end{array}$ & $\begin{array}{l}17,623 \\
14,502\end{array}$ & $\begin{array}{l}8,585 \\
2,454\end{array}$ & (b) & (b) & $\begin{array}{l}17,623 \\
29,080\end{array}$ & $\begin{array}{l}8,585 \\
8,846\end{array}$ \\
\hline Johnson (1), Linn (2) & 3 & 13,108 & 10,652 & 4,572 & 2,051 & (b) & (b) & 17,680 & 12,703 \\
\hline $\begin{array}{l}\text { Lee (1), Des Moines (3) } \\
\text { Marion (1), Story (1), Tama (1) }\end{array}$ & 4 & 1,241 & $\begin{array}{l}1,130 \\
3045\end{array}$ & 5,932 & 1,793 & (c) & (c) & 7,173 & 2,9 \\
\hline $\begin{array}{l}\text { Marion (1), Story (1), Tama (1) } \\
\text { Muscatine (4), Scott (1) }\end{array}$ & $\begin{array}{l}3 \\
5\end{array}$ & $\begin{array}{l}5,124 \\
8,885\end{array}$ & $\begin{array}{l}3,045 \\
4,930\end{array}$ & $\begin{array}{l}\text { (c) } \\
28,703\end{array}$ & $\begin{array}{c}(c) \\
10,657\end{array}$ & $\begin{array}{l}\text { (c) } \\
28,757\end{array}$ & 29,050 & $\begin{array}{r}5,124 \\
66,345\end{array}$ & $\begin{array}{r}3,045 \\
44,637\end{array}$ \\
\hline Polk (6) & 6 & 59,401 & 32,080 & 39,644 & 12,218 & 2,640 & & 101,685 & 45,4 \\
\hline $\begin{array}{l}\text { Total commercial, } 1933 \\
\text { Total noncommercial } 1\end{array}$ & 60 & 218,402 & 113,711 & $\begin{array}{r}340,069 \\
96,550\end{array}$ & $\begin{array}{r}112,173 \\
6,985\end{array}$ & 80,538 & 47,172 & $\begin{array}{r}639,009 \\
97,704\end{array}$ & $\begin{array}{r}273,05 \\
7,89\end{array}$ \\
\hline lotal & & 219,556 & 114,618 & 436,619 & 119,158 & 80,538 & 47,172 & 736,713 & 280,948 \\
\hline & 78 & 233,734 & 114,525 & 805,467 & 198,891 & & & $1,039,201$ & 313,316 \\
\hline
\end{tabular}

a Includes molding, blast, engine, filter, and railroad ballast sand.

b Included with paving sand,

c Included with structural sand. 
TABLE XXXIII

Total Production of Sand and Gravel in Iover in 1933 - Gravel

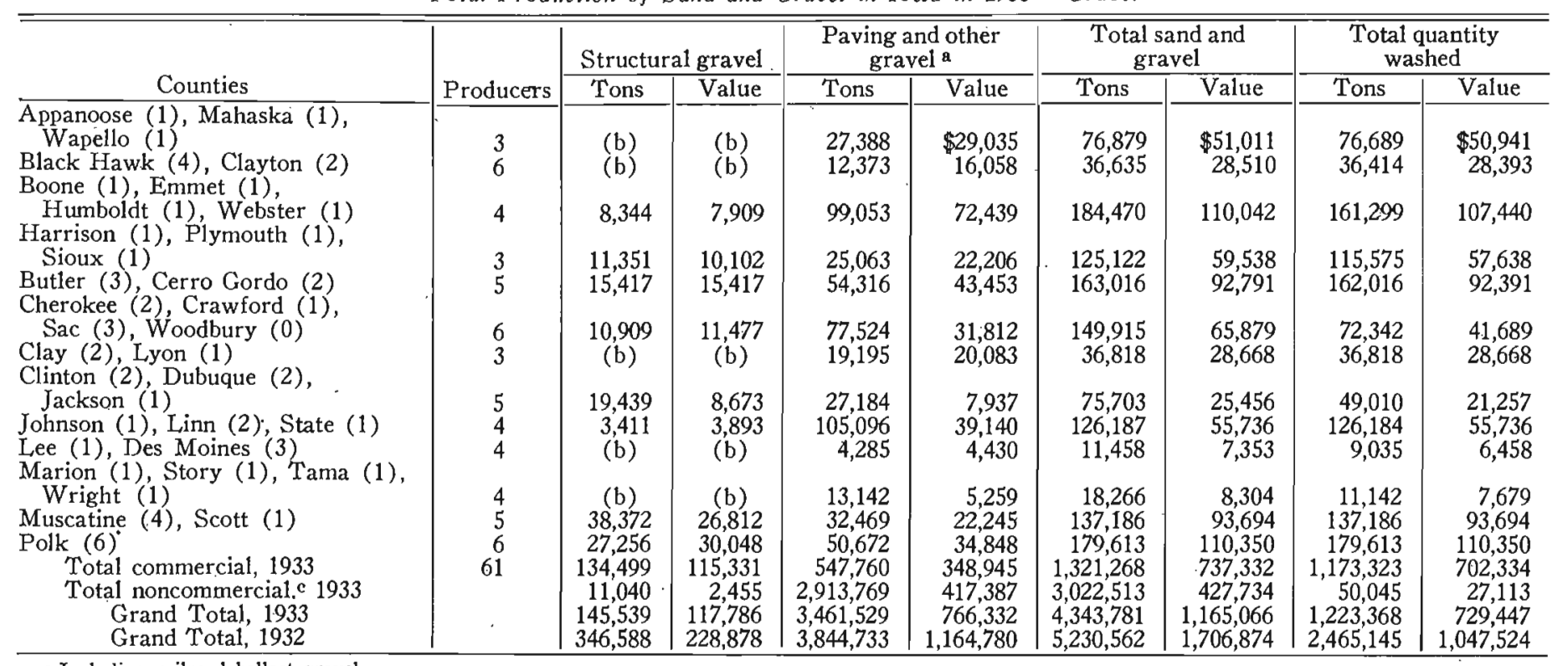

a Including railroad ballast gravel.

c Includes railroad ballast rravel. 
TABLE XXXIV

Total Production of Sand and Gravel in Iowa in 1934 - Sand

\begin{tabular}{|c|c|c|c|c|c|c|c|c|c|}
\hline \multirow[b]{2}{*}{ Counties } & \multirow[b]{2}{*}{ Producers } & \multicolumn{2}{|c|}{ Structural sand } & \multicolumn{2}{|c|}{ Paving sand } & \multicolumn{2}{|c|}{ Other sand a } & \multicolumn{2}{|c|}{ Total sand } \\
\hline & & Tons & Value & Tons & Value & Tons & Value & Tons & Value \\
\hline $\begin{array}{l}\text { Appanoose (1), Lee (1), } \\
\text { Des Moines (1), State (0) }\end{array}$ & $\begin{array}{l}3 \\
5\end{array}$ & $\begin{array}{l}5,908 \\
8758\end{array}$ & $\begin{array}{r}\$ 2,574 \\
3755\end{array}$ & $\begin{array}{r}5,356 \\
30701\end{array}$ & $\$ 2,302$ & (b) & (b) & $\begin{array}{l}11,264 \\
46790\end{array}$ & $\$ 4,876$ \\
\hline Boone (1), Emmet (1), & 5 & & & $30, / 01$ & 15,740 & 7,540 & $\$ 4,408$ & 40,799 & 23,909 \\
\hline $\begin{array}{l}\text { Humboldt (1), Webster (1) } \\
\text { Clayton (2), Dubuque (2), }\end{array}$ & 4 & 23,297 & 11,759 & 22,049 & 7,680 & (c) & (c) & 45,346 & 19,439 \\
\hline Jackson (1) & 5 & 69,655 & 24,728 & 34,773 & 8,446 & 18,873 & .10,497 & 123,301 & 43,671 \\
\hline Clinton (2), Scott (1) & 3 & 25,428 & 13,615 & 14,259 & 4,785 & (c) & (c) & 39,687 & 18,400 \\
\hline $\begin{array}{l}\text { Linn (1), Mahaska (1), Tama (1) } \\
\text { Lyon (2) Clay (1) Sioux (2) }\end{array}$ & $\begin{array}{l}3 \\
5\end{array}$ & $\begin{array}{l}26,241 \\
44,349\end{array}$ & $\begin{array}{l}17,510 \\
12,231\end{array}$ & $\begin{array}{l}10,960 \\
79,267\end{array}$ & $\begin{array}{r}4,675 \\
29,500\end{array}$ & $\begin{array}{l}\text { (c) } \\
\text { (b) }\end{array}$ & $\begin{array}{l}\text { (c) } \\
\text { (b) }\end{array}$ & $\begin{array}{r}37,201 \\
123,616\end{array}$ & $\begin{array}{l}22,185 \\
41,731\end{array}$ \\
\hline Muscatine (3) & 3 & 41,455 & 21,579 & 42,005 & 21,799 & 32,903 & 30,377 & 116,363 & 73,755 \\
\hline Polk (6) & 6 & 64,471 & 26,441 & 50,249 & 14,072 & 9,496 & 4,629 & 124,216 & 45,142 \\
\hline Sac (3), Cherokee (1), Plymouth (1) & $\begin{array}{l}5 \\
3\end{array}$ & $\begin{array}{l}20,480 \\
41,409\end{array}$ & $\begin{array}{r}7,367 \\
24,104\end{array}$ & $\begin{array}{l}45,085 \\
25,617\end{array}$ & $\begin{array}{l}20,352 \\
10300\end{array}$ & 2,060 & $\begin{array}{r}964 \\
1264\end{array}$ & $\begin{array}{l}67,625 \\
70287\end{array}$ & $\begin{array}{l}28,683 \\
35,668\end{array}$ \\
\hline $\begin{array}{l}\text { Wapello } \\
\text { Total commercial, } 1934\end{array}$ & 45 & 371,451 & 165,663 & 360,321 & 139,657 & 73,933 & 52,199 & 805,705 & $\begin{array}{r}55,000 \\
357,519\end{array}$ \\
\hline Total noncommercial, 1934 & & 11,565 & 5,742 & 102,579 & 12,698 & 2,109 & 125 & 116,253 & 18,565 \\
\hline Grand Total, 1934 & & 383,016 & 171,405 & 462,900 & 152,355 & 76,042 & 52,324 & 921,958 & 376,084 \\
\hline Grand Total, 1933 & & 219,556 & 114,618 & 436,619 & 119,158 & 80,538 & 47,172 & 736,713 & 280,948 \\
\hline
\end{tabular}

a Includes molding, blast, engine, filter, and railroad ballast sand.

b Included with structural sand.

cincluded with paving sand. 
TABLE XXXV

Total Production of Sand and Gravel in Iowa in 1934-Gravel

\begin{tabular}{|c|c|c|c|c|c|c|c|c|c|}
\hline \multirow[b]{2}{*}{ Counties } & \multirow[b]{2}{*}{ Producers } & \multicolumn{2}{|c|}{ Structural gravel } & \multicolumn{2}{|c|}{$\begin{array}{c}\text { Paving and other } \\
\text { gravel }\end{array}$} & \multicolumn{2}{|c|}{$\begin{array}{l}\text { Total sand and } \\
\text { gravel }\end{array}$} & \multicolumn{2}{|c|}{$\begin{array}{l}\text { Total quantity } \\
\text { washed }\end{array}$} \\
\hline & & Tons & Value & Tons & Value & Tons & Value & Tons & Value \\
\hline $\begin{array}{l}\text { Appanoose (0), Lee (1), Des Moines } \\
\text { (1), State (2) } \\
\text { Black Hawk (3), Butler (1) } \\
\text { Boone (1), Emmet (1), Humboldt (1), }\end{array}$ & $\begin{array}{l}4 \\
4\end{array}$ & $\begin{array}{l}8,841 \\
9,754\end{array}$ & $\begin{array}{r}\$ 6,221 \\
7,806\end{array}$ & $\begin{array}{r}133,150 \\
12,086\end{array}$ & $\begin{array}{r}\$ 28,600 \\
11,951\end{array}$ & $\begin{array}{r}153,255 \\
68,639\end{array}$ & $\begin{array}{r}\$ 39,697 \\
43,726\end{array}$ & $\begin{array}{l}98,695 \\
67,764\end{array}$ & $\begin{array}{r}\$ 33,597 \\
43,376\end{array}$ \\
\hline $\begin{array}{l}\text { Webster (2) } \\
\text { Clayton (2), Dubuque (2), Jackson (1) } \\
\text { Clinton (2), Scott (1) } \\
\text { Linn (0), Mahaska (1), Tama (1), }\end{array}$ & $\begin{array}{l}5 \\
5 \\
3\end{array}$ & $\begin{array}{l}11,764 \\
53,777 \\
47,178\end{array}$ & $\begin{array}{l}11,640 \\
43,119 \\
25,688\end{array}$ & $\begin{array}{c}31,786 \\
40,864 \\
\text { (a) }\end{array}$ & $\begin{array}{c}24,001 \\
26,843 \\
\text { (a) }\end{array}$ & $\begin{array}{r}88,896 \\
217,942 \\
86,865\end{array}$ & $\begin{array}{r}55,080 \\
113,633 \\
44,088\end{array}$ & $\begin{array}{r}83,284 \\
165,827 \\
82,093\end{array}$ & $\begin{array}{r}54,486 \\
104,037 \\
43,577\end{array}$ \\
\hline $\begin{array}{l}\text { Van Buren (1) } \\
\text { Lyon (2), Clay (1), Sioux (2) } \\
\text { Muscatine (4) } \\
\text { Polk (6). } \\
\text { Sac (3), Cherokee (1), Plymouth (1) } \\
\text { Wapello (1), Cerro Gordo (2) }\end{array}$ & $\begin{array}{l}3 \\
5 \\
4 \\
6 \\
5 \\
3\end{array}$ & $\begin{array}{c}(\mathrm{b}) \\
26,132 \\
16,965 \\
37,574 \\
33,532 \\
16,604\end{array}$ & $\begin{array}{l}(\mathrm{b}) \\
20,576 \\
13,894 \\
47,494 \\
23,969 \\
18,306\end{array}$ & $\begin{array}{l}29,164 \\
68,694 \\
41,740 \\
69,840 \\
82,120 \\
15,022\end{array}$ & $\begin{array}{l}20,356 \\
56,600 \\
34,545 \\
44,845 \\
46,463 \\
15,397\end{array}$ & $\begin{array}{r}65,365 \\
218,442 \\
175,068 \\
231,630 \\
183,277 \\
101,913\end{array}$ & $\begin{array}{r}42,541 \\
118,907 \\
122,194 \\
137,481 \\
99,115 \\
69,371\end{array}$ & $\begin{array}{r}57,465 \\
207,621 \\
175,068 \\
231,630 \\
179,899 \\
101,913\end{array}$ & $\begin{array}{r}41,341 \\
117,560 \\
122,194 \\
137,481 \\
98,115 \\
69,371\end{array}$ \\
\hline $\begin{array}{l}\text { son (2), Mitchell (1), O'Brien (1) } \\
\text { Total commercial, 1934 } \\
\text { Total noncommercialc, } 1934 \\
\text { Grand Total, 1934 } \\
\text { Grand Total, 1933 }\end{array}$ & $\begin{array}{r}6 \\
49\end{array}$ & $\begin{array}{r}4,178 \\
266,299 \\
15,918 \\
282,217 \\
145,539\end{array}$ & $\begin{array}{r}1,595 \\
220,308 \\
4,443 \\
224,751 \\
117,878\end{array}$ & $\begin{array}{r}39,260 \\
563,726 \\
2,581,461 \\
3,145,187 \\
3,461,529\end{array}$ & $\begin{array}{r}8,345 \\
317,946 \\
475,219 \\
793,165 \\
766,332\end{array}$ & $\begin{array}{r}43,438 \\
1,635,730 \\
2,713,632 \\
4,349,362 \\
4,343,781\end{array}$ & $\begin{array}{r}9,940 \\
895,773 \\
498,227 \\
1,394,000 \\
1,165,066\end{array}$ & $\begin{array}{r}2,380 \\
1,453,639 \\
141,520 \\
1,595,159 \\
1,223,368\end{array}$ & $\begin{array}{r}1,340 \\
866,475 \\
61,991 \\
928,466 \\
729,447\end{array}$ \\
\hline
\end{tabular}

a Included with structural gravel.

c Includes railroad ballast gravel. 
TABLE XXXVI

Total Production of Sand and Gravel in Iozera in 1935-Sand

\begin{tabular}{|c|c|c|c|c|c|c|c|c|c|}
\hline \multirow[b]{2}{*}{ Counties } & \multirow[b]{2}{*}{ Producers } & \multicolumn{2}{|c|}{ Structural sand } & \multicolumn{2}{|c|}{ Paving sand } & \multicolumn{2}{|c|}{ Other sand ${ }^{a}$} & \multicolumn{2}{|c|}{ Total sand } \\
\hline & & Tons & Value & Tons & Value & Tons & Value & Tons & Value \\
\hline Black Hawk (4), Linn (1) & $\begin{array}{l}5 \\
3\end{array}$ & $\begin{array}{l}58,779 \\
18,488\end{array}$ & $\begin{array}{r}\$ 38,215 \\
8,045\end{array}$ & $\begin{array}{l}\text { (b) } \\
40,131\end{array}$ & $\begin{array}{c}(\mathrm{b}) \\
\$ 14,734\end{array}$ & (b) & $\begin{array}{l}\text { (b) } \\
\text { (b) }\end{array}$ & $\begin{array}{l}58,779 \\
58,619\end{array}$ & $\begin{array}{r}\$ 38,215 \\
22,779\end{array}$ \\
\hline $\begin{array}{l}\text { Clayton (1), Dubuque (2), } \\
\text { Jackson (1), Clinton (1) } \\
\text { Des Moines (1). Mahaska }\end{array}$ & 5 & 48,412 & 21,211 & 74,766 & 42,685 & (c) & (c) & 123,178 & 63,896 \\
\hline $\begin{array}{l}\text { Wapello (1), Lee (2) } \\
\text { Humboldt (1), Webster (1), }\end{array}$ & 5 & 52,145 & 27,209 & 28,165 & 10,608 & (b) & (b) & 79,310 & 37,817 \\
\hline $\begin{array}{l}\text { Boone (1), Wright (0) } \\
\text { Lyon (2), Sioux }(2) \\
\text { Mitchell }(0) \text {, Butler }(1) \text {, }\end{array}$ & $\begin{array}{l}3 \\
4\end{array}$ & $\begin{array}{l}35,873 \\
45,200\end{array}$ & $\begin{array}{l}17,288 \\
13,100\end{array}$ & $\begin{array}{l}\text { (b) } \\
76,516\end{array}$ & $\begin{array}{c}\text { (b) } \\
26,455\end{array}$ & (b) & (b) & $\begin{array}{r}35,873 \\
121,716\end{array}$ & $\begin{array}{l}17,288 \\
39,555\end{array}$ \\
\hline Tama (1), Cerro Gordo (2) & 4 & 46,656 & 23,946 & $23,650^{-}$ & 11,200 & (c) & (c) & 70,306 & 35,146 \\
\hline Muscatine (3), Scott (1) & 4 & 33,151 & 16,659 & 29,392 & 12,688 & 61,724 & 50,052 & 124,267 & 79,399 \\
\hline Polk (5) & 5 & 66,584 & 31,544 & 49,886 & 18,374 & (c) & (c) & 116,470 & 49,918 \\
\hline $\begin{array}{l}\text { Sac (3), Harrison }(0) \text {, State }(0) \\
\text { Total commercial, } 1935\end{array}$ & $\begin{array}{r}3 \\
41\end{array}$ & $\begin{array}{r}6,625 \\
410,913\end{array}$ & $\begin{array}{r}1,746 \\
198,963\end{array}$ & $\begin{array}{r}16,625 \\
339,131\end{array}$ & $\begin{array}{r}4,723 \\
141,467\end{array}$ & $\begin{array}{c}\text { (c) } \\
61,724\end{array}$ & $\begin{array}{c}(c) \\
50,052\end{array}$ & $\begin{array}{r}23,250 \\
811,768\end{array}$ & $\begin{array}{r}6,469 \\
390,482\end{array}$ \\
\hline Total noncommercial, 1935 & & 11,400 & $\begin{array}{r}1,440 \\
200403\end{array}$ & 167,295 & 21,740 & 61724 & 50052 & 178,695 & 23,180 \\
\hline Grand Total, 1934 & & $\begin{array}{l}422,313 \\
383,016\end{array}$ & $\begin{array}{l}200,403 \\
171,405\end{array}$ & $\begin{array}{l}506,426 \\
462,900\end{array}$ & $\begin{array}{l}163,207 \\
152,355\end{array}$ & $\begin{array}{l}61,724 \\
76,042\end{array}$ & $\begin{array}{l}50,052 \\
52,324\end{array}$ & $\begin{array}{l}990,463 \\
921,958\end{array}$ & $\begin{array}{l}413,002 \\
376,084\end{array}$ \\
\hline
\end{tabular}

a Includes molding, cutting and grinding, engine, filter, and railroad ballast sand.

b Included under structural sand.
c Included under paving sand. 
TABLE XXXVII

Total Production of Sand and Gravel in Iowa in 1935 - Gravel

\begin{tabular}{|c|c|c|c|c|c|c|c|c|c|}
\hline \multirow[b]{2}{*}{ Counties } & \multirow[b]{2}{*}{ Producers } & \multicolumn{2}{|c|}{ Structural gravel a } & \multicolumn{2}{|c|}{$\begin{array}{c}\text { Paving and other } \\
\text { gravel }\end{array}$} & \multicolumn{2}{|c|}{$\begin{array}{c}\text { Total sand and } \\
\text { gravel }\end{array}$} & \multicolumn{2}{|c|}{$\begin{array}{l}\text { Total quantity } \\
\text { washed. }\end{array}$} \\
\hline & & Tons & Value & Tons & Value & Tons & Value & Tons & Value \\
\hline $\begin{array}{l}\text { Black Hawk (4), Linn }(0) \\
\text { Cherokee (1), Clay (1), Emmet (1) }\end{array}$ & $\begin{array}{l}4 \\
3\end{array}$ & $\begin{array}{c}10,277 \\
\text { (c) }\end{array}$ & $\begin{array}{l}\$ 10,945 \\
(\mathrm{c})\end{array}$ & $\begin{array}{c}\text { (b) } \\
66,745\end{array}$ & $\begin{array}{c}\text { (b) } \\
56,638\end{array}$ & $\begin{array}{r}60,056 \\
125,364\end{array}$ & $\begin{array}{r}\$ 49,160 \\
79,417\end{array}$ & $\begin{array}{r}51,556 \\
125,364\end{array}$ & $\begin{array}{l}\$ 34,436 \\
79,417\end{array}$ \\
\hline $\begin{array}{l}\text { Clayton (0), Dubuque }(2) \\
\text { Jackson (1) Clinton (1) }\end{array}$ & 4 & 110346 & 57540 & (b) & (b) & 233.524 & 121436 & 2335240 & 1214.360 \\
\hline Des Moines (1), Mahaska (1), & $\mathbf{T}$ & 110,340 & $37,5+0$ & (2000 & (0) & & & & $1,2+7,006$ \\
\hline $\begin{array}{l}\text { Wapello (1), Lee (0) } \\
\text { Humboldt (1), Webster (1), }\end{array}$ & 3 & (c) & (c), & 33,902 & 33,667 & 113,212 & 71,484 & 106,852 & 69,254 \\
\hline $\begin{array}{l}\text { Boone (1), Wright (1) } \\
\text { Lyon (2), Sioux (2) } \\
\text { Mitchell (2), Butler (1), }\end{array}$ & $\begin{array}{l}4 \\
4\end{array}$ & $\begin{array}{l}11,724 \\
25,800\end{array}$ & $\begin{array}{r}8,134 \\
20,720\end{array}$ & $\begin{array}{l}31,913 \\
70,595\end{array}$ & $\begin{array}{l}24,994 \\
55,105\end{array}$ & $\begin{array}{r}79,510 \\
218,111\end{array}$ & $\begin{array}{r}50,416 \\
115,380\end{array}$ & $\begin{array}{r}73,680 \\
205,111\end{array}$ & $\begin{array}{r}49,802 \\
112,240\end{array}$ \\
\hline Tama (1), Cerro Gordo (2) & 6 & $\cdot 24,793$ & 25,160 & 140,356 & 47,329 & 235,455 & 107,635 & 223,180 & 105,565 \\
\hline $\begin{array}{l}\text { Muscatine (3), Scott }(0) \\
\text { Polk (5) }\end{array}$ & 3 & $\begin{array}{c}(c) \\
50720\end{array}$ & $\begin{array}{l}\text { (c) } \\
50067\end{array}$ & $\begin{array}{l}133,430 \\
59120\end{array}$ & $\begin{array}{r}93,019 \\
45041\end{array}$ & $\begin{array}{l}257,697 \\
226310\end{array}$ & $\begin{array}{l}172,418 \\
154026\end{array}$ & $\begin{array}{l}215,358 \\
226,310\end{array}$ & 150,778 \\
\hline Sac (3), Harrison (1), State (3) & 7 & 19,197 & 6,569 & 455,635 & 149,699 & 498,082 & 162.737 & 339,182 & 136,237 \\
\hline Total commercial, 1935 & 43 & 252,857 & 189,035 & 991,696 & $\begin{array}{l}505,492 \\
648 ? 37\end{array}$ & $2,056,321$ & $1,085,009$ & $\begin{array}{l}3,901,833 \\
63,324\end{array}$ & $2,107,014$ \\
\hline $\begin{array}{l}\text { Total noncommercial,a } 1935 \\
\text { Grand Total, } 1935\end{array}$ & & 254,117 & 189,460 & $4,488,162$ & $1,153,729$ & $5,732,742$ & $1,756,851$ & $3,965,157$ & $2,131,906$ \\
\hline Grand Total, 1934 & & 282,217 & 224,751 & $3,145,187$ & 793,165 & $4,349,362$ & $1,394,000$ & $1.595,159$ & 928,466 \\
\hline
\end{tabular}


TABLE XXXVIII

Total Production of Sand and Gravel in Iowa in 1936-Sand

\begin{tabular}{|c|c|c|c|c|c|c|c|c|c|}
\hline \multirow[b]{2}{*}{ Counties } & \multirow[b]{2}{*}{ Producers } & \multicolumn{2}{|c|}{ Structural sand } & \multicolumn{2}{|c|}{ Paving sand } & \multicolumn{2}{|c|}{ Other sand ${ }^{a}$} & \multicolumn{2}{|c|}{ Total sand } \\
\hline & & Tons & Value & Tons & Value & Tons & Value & Tons & Value \\
\hline Black Hawk (4), Linn (1) & 5 & 31,296 & $\$ 19,821$ & 13,551 & $\$ 5,873$ & 16,269 & $\$ 8,962$ & 61,116 & $\$ 34,656$ \\
\hline Boone (1), Dallas (0), Polk (4) & 5 & 86,539 & 50,633 & 56,918 & 19,252 & (c) & (c) & 143,457 & 69,885 \\
\hline Humboldt (1), Webster (0) & 3 & 63,347 & 34,864 & 81,859 & 40,503 & (c) & (c) & 145,206 & 75,367 \\
\hline Clayton (1), Dubuque (2), & & & & & & & & & \\
\hline $\begin{array}{l}\text { Clinton (1), Jackson (2) } \\
\text { Emmet (1), O'Brien (0), Clay (1), }\end{array}$ & 6 & 30,568 & 12,734 & 66,210 & 38,753 & (c) & (c) & 96,778 & 51,487 \\
\hline Cherokee (1) & 3 & 114,325 & 44,340 & (b) & (b) & (b) & (b) & 114,325 & 44,340 \\
\hline $\begin{array}{l}\text { Lyon (2), Sioux (1), State (1) } \\
\text { Mahaska (1), Wapello (1), Lee (2) }\end{array}$ & $\begin{array}{l}4 \\
4\end{array}$ & $\begin{array}{c}(c) \\
48,090\end{array}$ & $\begin{array}{c}(c) \\
29,763\end{array}$ & $\begin{array}{r}118,312 \\
47,943\end{array}$ & $\begin{array}{l}56,100 \\
19,922\end{array}$ & (c) & (c) & $\begin{array}{r}118,312 \\
96,033\end{array}$ & $\begin{array}{l}56,100 \\
49,685\end{array}$ \\
\hline Mitchell (0), Butler (2), Tama (1) & 3 & 17,252 & 11,926 & (b) & (b) & & & 17,252 & 11,926 \\
\hline $\begin{array}{l}\text { Sac (2), Harrison (1) } \\
\text { Scott (1), Muscatine (3), }\end{array}$ & 3 & 63,615 & 20,286 & & (b) & (b) & (b) & 63,615 & 20,286 \\
\hline Des Moines (1) & 5 & 119,933 & 53,665 & 69,536 & 26,395 & 46,635 & 40,325 & 236,104 & 120,385 \\
\hline $\begin{array}{l}\text { Total commercial, } 1936 \\
\text { Total noncommercial } 1936\end{array}$ & 41 & $\begin{array}{r}574,965 \\
7988\end{array}$ & 278,032 & $\begin{array}{r}454,329 \\
70,165\end{array}$ & 206,798 & 62,904 & 49,287 & $\begin{array}{r}1,092,198 \\
78,153\end{array}$ & 534,117 \\
\hline Grand Total, 1936 & & 582,953 & 280,145 & 524,494 & 222,282 & 62,904 & 49,287 & $1,170,351$ & 551,714 \\
\hline Grand Total, 1935 & & 422,313 & 200,403 & 506,426 & 163,207 & 61,724 & 50,052 & 990,463 & 413,662 \\
\hline
\end{tabular}


TABLE XXXIX

Total Production of Sand and Gravel in Iowe in 1936-Gravel

\begin{tabular}{|c|c|c|c|c|c|c|c|c|c|}
\hline \multirow[b]{2}{*}{ Counties } & \multirow[b]{2}{*}{ Producers } & \multicolumn{2}{|c|}{ Structural gravel a } & \multicolumn{2}{|c|}{$\begin{array}{l}\text { Paving and other } \\
\text { gravel }\end{array}$} & \multicolumn{2}{|c|}{$\begin{array}{l}\text { Total sand and } \\
\text { gravel }\end{array}$} & \multicolumn{2}{|c|}{$\begin{array}{l}\text { Total quantity } \\
\text { washed }\end{array}$} \\
\hline & & Tons & Value & Tons & Value & Tons & Value & Tons & Value \\
\hline $\begin{array}{l}\text { Black Hawk (4), Linn (0) } \\
\text { Boone (1), Dallas (1), Polk (4) } \\
\text { Cerro Gordo (2), Wright (2) }\end{array}$ & $\begin{array}{l}4 \\
6\end{array}$ & $\begin{array}{l}16,175 \\
62,867\end{array}$ & $\begin{array}{r}\$ 18,710 \\
77,192\end{array}$ & $\begin{array}{c}\text { (b) } \\
189,313\end{array}$ & $\begin{array}{c}\text { (b) } \\
79,281\end{array}$ & $\begin{array}{r}77,291 \\
395,637\end{array}$ & & $\begin{array}{r}77,291 \\
395,637\end{array}$ & $\begin{array}{l}\$ 17,366 \\
226,358\end{array}$ \\
\hline $\begin{array}{l}\text { Humboldt (1), Webster (3)' } \\
\text { Clayton (0) Dubuque (2) }\end{array}$ & 8 & 37,952 & 53,611 & 114,356 & 97,702 & 297,514 & 226,680 & 261,820 & 222,893 \\
\hline $\begin{array}{l}\text { Jackson (1), Clinton (2) } \\
\text { Emmet (1), O'Brien (1), }\end{array}$ & 5 & 101,782 & 53,734 & (b) & (b) & 198,560 & 105,221 & 198,560 & 105,221 \\
\hline $\begin{array}{l}\text { Clay (1), Cherokee (1) } \\
\text { L,yon (1), Sioux (1), State (2) } \\
\text { Mahaska (1), Wapello (1), Lee (2) } \\
\text { Mitchell (1), Butler (1), Tama (1) } \\
\text { Sac (2), Harrison (2) } \\
\text { Scott (0), Muscatine (3), }\end{array}$ & $\begin{array}{l}4 \\
4 \\
4 \\
3 \\
4\end{array}$ & $\begin{array}{l}(\mathrm{c}) \\
(\mathrm{c}) \\
38,143 \\
(\mathrm{c}) \\
(\mathrm{c})\end{array}$ & $\begin{array}{l}(\mathrm{c}) \\
(\mathrm{c}) \\
41,195 \\
\text { (c) } \\
\text { (c) }\end{array}$ & $\begin{array}{l}169,867 \\
395,714 \\
\text { (b) } \\
16,798 \\
325,521\end{array}$ & $\begin{array}{l}136,068 \\
109,364 \\
\text { (b) } \\
14,128 \\
139,888\end{array}$ & $\begin{array}{r}284,192 \\
514,026 \\
134,176 \\
34,050 \\
389,136\end{array}$ & $\begin{array}{r}180,408 \\
165,464 \\
90,880 \\
26,054 \\
160,174\end{array}$ & $\begin{array}{r}256,303 \\
232,826 \\
134,176 \\
30,930 \\
386,896\end{array}$ & $\begin{array}{r}178,409 \\
144,114 \\
90,880 \\
24,512 \\
160,014\end{array}$ \\
\hline $\begin{array}{l}\text { Des Moines (1) } \\
\text { Total commercial, } 1936 \\
\text { Total noncommercial,d } 1936 \\
\text { Grand Total, } 1936 \\
\text { Grand Total, } 1935\end{array}$ & $\begin{array}{r}4 \\
46\end{array}$ & $\begin{array}{c}(c) \\
256,919 \\
32,906 \\
289,825 \\
254,117\end{array}$ & $\begin{array}{r}(c) \\
244,442 \\
5,460 \\
249,902 \\
189,460\end{array}$ & $\begin{array}{r}177,623 \\
1,389,192 \\
3,444,616 \\
4,833,808 \\
4,488,162\end{array}$ & $\begin{array}{r}134,817 \\
711,248 \\
535,418 \\
1,246,666 \\
1,153,729\end{array}$ & $\begin{array}{r}413,727 \\
2,738,309 \\
3,555,675 \\
6,293,984 \\
5,732,742\end{array}$ & $\begin{array}{r}255,202 \\
1,489,807 \\
558,475 \\
2,048,282 \\
1,756,851\end{array}$ & $\begin{array}{r}395,926 \\
2,370,365 \\
77,697 \\
2,448,062 \\
3,965,157\end{array}$ & $\begin{array}{r}322,322 \\
1,492,089 \\
29,372 \\
1,521,461 \\
2,131,906\end{array}$ \\
\hline
\end{tabular}

a Structural gravel includes some paving gravel and paving gravel includes some structural gravel.

b Included under structural gravel.

d Includes railroad ballast gravel. 
TABLE XL

Total Production of Sand and Gravel in Iowa in 1937 - Sand

\begin{tabular}{|c|c|c|c|c|c|c|c|c|c|}
\hline \multirow[b]{2}{*}{ Counties } & \multirow[b]{2}{*}{ Producers } & \multicolumn{2}{|c|}{ Structural sand } & \multicolumn{2}{|c|}{ Paving sand } & \multicolumn{2}{|c|}{ Other sanda } & \multicolumn{2}{|c|}{ Total sand } \\
\hline & & Tons & Value & Tons & Value & Tons & Value & Tons & Value \\
\hline Black Hawk (5), Winneshiek (1), & & & & & & & $\$ 51,400$ & ד070070 & \\
\hline Cerro Gordo (2), Mitchell (0), & 8 & 48,504 & $\$ 33,2 / 0$ & (D) & (D) & 74,403 & $\$ 51,409$ & 122,907 & $\$ 04,0 / 9$ \\
\hline Butler (2) & 4 & 50,392 & 36,871 & 49,309 & 34,601 & (c) & (c) & 99,701 & 71,472 \\
\hline $\begin{array}{l}\text { Crawford (0), Sac (2), Carroll (1), } \\
\text { Harrison (0) }\end{array}$ & 3 & 73,738 & 30,986 & (b) & (b) & & & 73,738 & 30,986 \\
\hline Dallas (0), Polk (3), State (1) & 4 & 102,862 & 46,830 & 65,377 & 30,774 & (c) & (c) & 168,239 & 77,604 \\
\hline $\begin{array}{l}\text { Dubuque (2), Jackson (1), Clinton (2) } \\
\text { Emmet (1), Cherokee (2), Buena }\end{array}$ & 5 & 33,405 & 22,332 & 55,910 & 17,427 & (c) & (c) & 89,315 & 39,759 \\
\hline Vista (1) Wricht (0) Wehcter & 4 & 32,393 & 13,976 & 97,578 & 34,663 & (c) & (c) & 129,971 & 48,639 \\
\hline $\begin{array}{l}\text { Humboldt } \\
(0) \text {, Boone (2) }\end{array}$ & 3 & 78,111 & 38,575 & (b) & (b) & (b) & (b) & 78,111 & 38,575 \\
\hline $\begin{array}{l}\text { Lee (2), Mahaska (1), Wapello (1) } \\
\text { Lyon (3), Sioux (1), O'Brien (0) }\end{array}$ & $\begin{array}{l}4 \\
4\end{array}$ & $\begin{array}{r}5,4,674 \\
191,727\end{array}$ & $\begin{array}{l}32,733 \\
71,254\end{array}$ & $\begin{array}{l}40,526 \\
\text { (b) }\end{array}$ & $\begin{array}{l}20,273 \\
\text { (b) }\end{array}$ & $\begin{array}{l}\text { (b) } \\
\text { (b) }\end{array}$ & (b) & $\begin{array}{r}95,200 \\
191,727\end{array}$ & $\begin{array}{l}53,006 \\
71,254\end{array}$ \\
\hline Muscatine (3), Des Moines (1), & & & & & & & & & \\
\hline Scott (1) & $:$ & 81,327 & 72,614 & 79,012 & 35,418 & (b) & (b) & $\begin{array}{r}160,339 \\
08,061\end{array}$ & $\begin{array}{r}108,032 \\
70,460\end{array}$ \\
\hline $\begin{array}{l}\text { Tama (2), Linn (1), Johnson (1) } \\
\text { Total commercial, } 1937\end{array}$ & $\begin{array}{r}4 \\
48\end{array}$ & $\begin{array}{r}02,900 \\
810,033\end{array}$ & 455,168 & 423,773 & $\begin{array}{r}14,142 \\
187,898\end{array}$ & 74,403 & 51,409 & $1,308,209$ & 694,475 \\
\hline Total noncommercial, 1937 & 7 & 20,204 & 2,419 & 13,391 & 5,901 & & & & 8,320 \\
\hline Grand Total, 1937 & 55 & 830,237 & 457,587 & 437,164 & 193,799 & 74,403 & 51,409 & $1,341,804$ & 702,795 \\
\hline Grand Total, 1936 & & & 280,1 & 524,494 & 222,282 & 62,904 & 49,287 & $1,170,351$ & 551,714 \\
\hline
\end{tabular}

a Includes molding, cutting and grinding, engine, filter, and railroad ballast sand.

b Included under structural sand. 
TABLE XII

Total Production of Sand and Gravel in Iovea in 1937 - Gravel

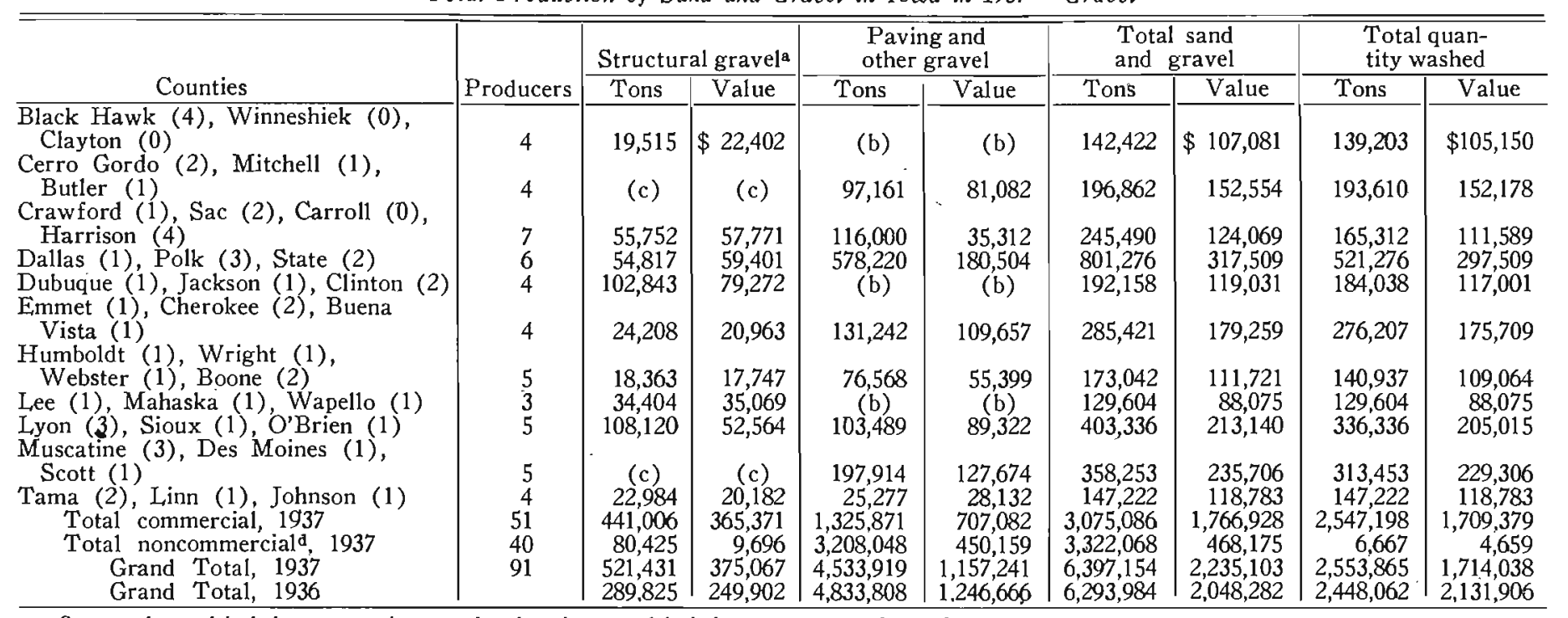

a Structural gravel includes some paving gravel and paving gravel includes some structural gravel.

b Included under. structural gravel.

d Includes railroad ballast gravel. 
TABLE XLII.

Total Production of Sand and Gravel in Iowa in 1938 - Sand

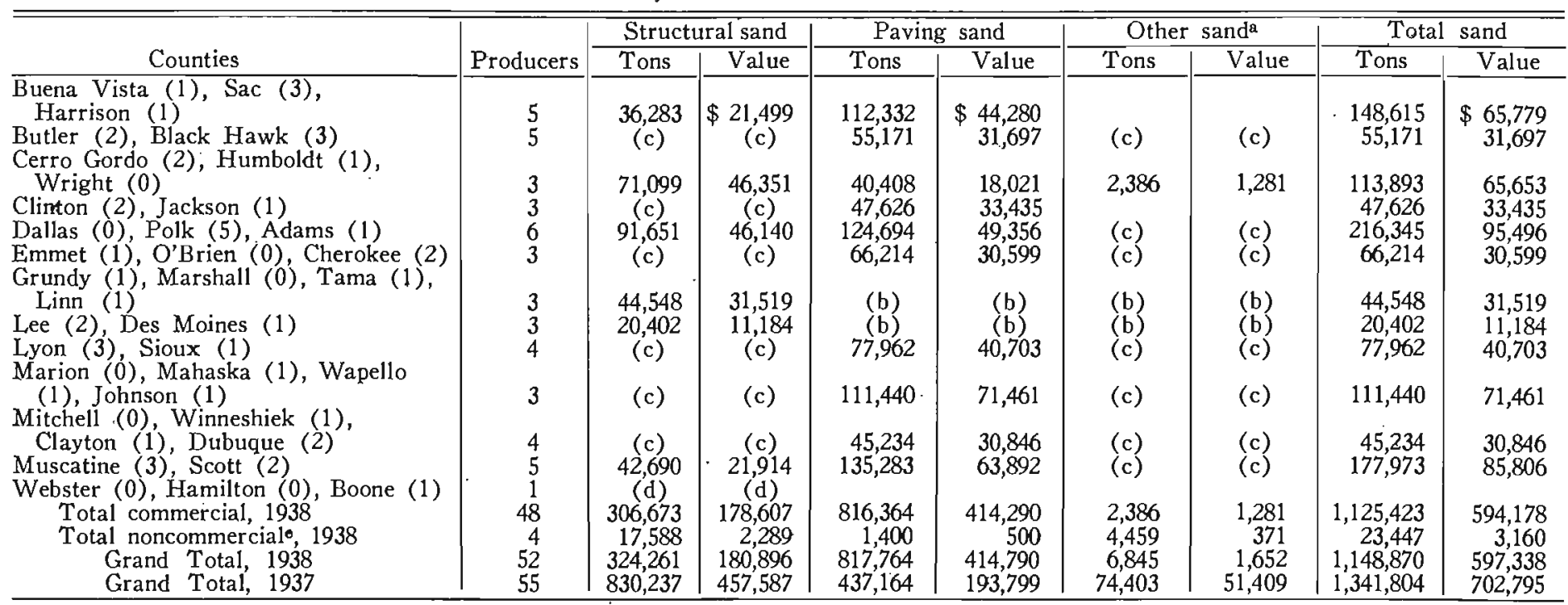

a Includes molding, cutting and grinding, engine, filter, and railroad ballast sand.

b Included under structural sanc.

Included under paving and other gravel.

e Includes railroad ballast sand. 
TABLE XLIII

Total Production of Sand and Gravel in Iowa in 1938-Gravel

\begin{tabular}{|c|c|c|c|c|c|c|c|c|c|}
\hline \multirow[b]{2}{*}{ Counties } & \multirow[b]{2}{*}{ Producers } & \multicolumn{2}{|c|}{ Structural gravel a } & \multicolumn{2}{|c|}{$\begin{array}{l}\text { Paving and other } \\
\text { gravel }\end{array}$} & \multicolumn{2}{|c|}{$\begin{array}{c}\text { Total sand and } \\
\text { gravel }\end{array}$} & \multicolumn{2}{|c|}{$\begin{array}{l}\text { Total quantity } \\
\text { washed }\end{array}$} \\
\hline & & Tons & Value & Tons & Value & Tons & Value & Tons & Value \\
\hline $\begin{array}{l}\text { Buena Vista (1), Sac (3), Harrison (2) } \\
\text { Butler (1), Black Hawk (2) }\end{array}$ & $\begin{array}{l}6 \\
3\end{array}$ & $\begin{array}{l}61,486 \\
\text { (c) }\end{array}$ & $\begin{array}{l}\$ 48,037 \\
\text { (c) }\end{array}$ & $\begin{array}{r}119,317 \\
15,515\end{array}$ & $\begin{array}{r}\$ 90,618 \\
18,927\end{array}$ & $\begin{array}{r}329,418 \\
70,686\end{array}$ & $\begin{array}{r}\$ 204,434 \\
50,624\end{array}$ & $\begin{array}{r}329,098 \\
63,903\end{array}$ & $\begin{array}{r}\$ 204,184 \\
47,954\end{array}$ \\
\hline Cerro Gordo (2), Humboldt (2), & 7 & 34.559 & 41.664 & 80 & 48,504 & 228,755 & 155.821 & 191.886 & 152010 \\
\hline Clinton (2), Jackson (1) & 3 & (c) & (c) & 84,9 & 44,173 & 132,608 & 77,608 & 132,608 & 77,608 \\
\hline Dallas (1), Polk (4), Adams (1) & 6 & 69,353 & 56,242 & 682,910 & 189,526 & 968,608 & 341,264 & 595,508 & 313,714 \\
\hline $\begin{array}{l}\text { (1), O'Brien (1), Cherokee (2) } \\
\text { (1), Marshall (1), }\end{array}$ & 4 & (c) & (c) & 107,379 & 64,280 & 173,593 & 94,879 & 149,793 & 93,179 \\
\hline Tama (1), Linn (1) & 4 & 62,252 & 22,082 & (b) & (b) & 106,800 & 53,601 & 54,042 & 43,731 \\
\hline Lee (2), Des Moines (1) & 3 & 9,740 & 9,065 & (b) & (b) & 30,142 & 20,249 & 30,142 & 20,249 \\
\hline Lyon (3), Sioux (1) & 4 & (c) & (c) & 104,648 & 78,986 & 182,610 & 119,689 & 182,610 & 119,689 \\
\hline $\begin{array}{l}\text { Marion (1), Mahaska (1), } \\
\text { Wapello (1), Johnson (1) }\end{array}$ & 4 & 74,359 & 62,828 & (b) & (b) & 185,799 & 134,289 & 185,799 & 134,289 \\
\hline $\begin{array}{l}\text { Mitchell (1), Winneshiek (1), } \\
\text { Clayton (0), Dubuque (1) }\end{array}$ & 3 & (c) & (c) & 25.277 & 7.687 & 70.511 & 385 & 103707 & 79452 \\
\hline ne (3), Scott (1) & 4 & (c) & (c) & 187,778 & 131,959 & 365,751 & 217,765 & 365,751 & 217,765 \\
\hline er (1), Hamilton (1), Boone (2) & 4 & (c) & (c) & 18,345 & 9,350 & 18,345 & 9,350 & 10,501 & 8,790 \\
\hline tal commercial, 1938 & 55 & 311,749 & 239,918 & $1,426,454$ & 684,010 & $2,863,626$ & 1,518 & $2,395,348$ & $1,512,614$ \\
\hline cial d, 1938 & 42 & $1,731,401$ & 520,195 & $2,375,812$ & 258,271 & $4,130,660$ & 781,626 & & 37,002 \\
\hline Gra & 97 & $2,043,150$ & 76 & $3,802,266$ & 942,281 & $6,994,2$ & $2,299,732$ & $2,465,864$ & $1,549,616$ \\
\hline Grand Total, 1 & 91 & 521,431 & & $4,533,919$ & $1,157,241$ & $6,397,154$ & $2,235,103$ & $2,553,865$ & $1,714,038$ \\
\hline
\end{tabular}

a Structural gravel includes some paving gravel and paving gravel includes some structural gravel.

b Included under structural gravel.

c Included under paving gravel. 


\section{Sand and Gravel Producers in Iowa}

Adams County

Maudlin Construction Co., 629 Ohio Street, Webster City, Iowa.

Robert E. Devereux, Route 3, Corning, Iowa.

Allamakee County

Northeastern Iowa Sand and Gravel Co., Harpers Ferry, Iowa.

Blackhawk County

Jay B. Bagenstos, LaPorte City, Iowa.

Concrete Materials Corp., 504 Lafayette Building, Waterloo, Iowa.

Martin Hanson \& Son, 1901 Commercial Street, Waterloo, Iowa. Waterloo Dredging Co., 85 W. Mullen, Waterloo, Iowa.

Waterloo Sand and Gravel Co., C. H. Werner, 335 Sheridan Road, Waterloo, Iowa.

Boone County

Munson \& Sons; Boone, Iowa.

Fraser Sand Company, c/o Otis Lumber Co., Boone, Iowa. Quarry at Fraser.

Markey River Sand Co., R. B. Markey, Boone, Iowa.

Buchanan County

Myron Baker, 1102 Fifth Avenue, N. E., Independence, Iowa.

Buena Vista County

LeGrand Limestone Co., 105 W. Madison Street, Chicago, Illinois, Quarry at Sioux Rapids.

L. L. Walton, Linn Grove, Iowa.

Butler County

Chas. Willeke \& Sons, Aplington, Iówa.

Waverly Gravel \& Tile Co., Shell Rock, Iowa.

Carroll County

Matt Lappe, Carroll, Iowa.

Cerro Gordo County

Clear Lake Sand \& Gravel Co., Clear Lake, Iowa.

Ideal Sand \& Gravel Co., Mason City, Iowa.

Cherokee County

Harris \& Loucks Gravel Co., Cherokee, Iowa.

Northwestern Gravel Co., Lake View, Iowa.

Shea Sand \& Gravel Co., Cherokee, Iowa. 


\section{Clayton County}

The Korite Corporation, 329 N. Milwaukee Street, Milwaukee, Wisconsin,

Langworthy Silica Co., 705 Federal Bank Building, Dubuque, Iowa.

Clinton County

Camanche Sand \& Gravel Co., Box 854, Davenport, Iowa.

Schneider Sand \& Gravel Co., Clinton, Iowa.

Crawford County

James Ballantine, Arion, Iowa.

Hannah Carlson, Kiron, Iowa.

Rogers Bros., Dunlap, Iowa.

Dallas County

Kaser Construction Co., Adel, Iowa.

Des Moines County

R. J. Dietlien, Burlington, Iowa.

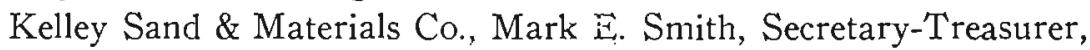
Burlington, Iowa.

Dubuque County

Lillie Coal Co., 510 Garfield Avenue, Dubuque, Iowa.

Molo Sand \& Gravel Co., 135 W. Fifth Street, Dubuque, Iowa.

Emmett County

Concrete Materials Corp., Lafayette Building, Waterloo, Iowa.

Fayette County

Clermont Brick \& Sand Co., Clermont, Iowa.

Grundy County

Ben Ankes, Wellsburg, Iowa.

Guthrie County

Ada Johnson, Guthrie Center, Iowa.

Hamilton County

James B. Weaver, National Bank \& Trust Co., Des Moines, Iowa.

Hardin County

Iowa Falls Sand \& Gravel Co., Iowa Falls, Iowa.

Harrison County

M. B. Musgrave, Woodbine, Iowa.

John Schumacher, Bancroft, Iowa.

Humboldt County

Concrete Materials Corporation, Waterloo, Iowa.

J. H. Tanck, Renwick, Iowa. 
Jackson County

Bellevue Sand \& Gravel Co., Att: A. C. Schneider, Bellevue, Iowa.

Johnson County

Central Sand \& Gravel Co., Iowa City, Iowa.

Hawkeye Material Co., Box 104, Iowa City, Iowa.

W. Stock, River Junction, Iowa.

Lee County

Joseph Jaeger, Fort Madison, Iowa.

Keokuk Sand Co., Foot of Bank Street, Keokuk, Iowa.

Linn County

Kings Crown Plaster Co., 98 First Avenue, W., Cedar Rapids, Iowa.

Lyon County

L. G. Everist, Inc., 2100 E. Fourth Street, Sioux City, Iowa. Pit at Klondike.

LeGrand Limestone Co., 105 W. Madison Street, Chicago, Illinois. Quarry at Rock Rapids.

Miller Sand \& Gravel Co., Box 101, Doon, Iowa.

Mahaska County

Concrete Materials Corp., Eddyville, Iowa.

Marion County

Harvey Sand \& Gravel Co., Harvey, Iowa.

Wilson Sand \& Gravel Co., Harvey, Iowa. Pit at Tracy.

Marshall County

Empire Sand \& Material Co., Lock Box 467, Marshalltown, Iowa. Pit at Keller.

LeGrand Limestone Co., Chicago, Illinois.

Sam Wright, New Providence, Iowa. Pit at Zearing.

Mitchell County

Falk \& Litzelman, St. Ansgar, Iowa. Pit at Osage.

Irvin C. Wheeler, McIntire, Iowa.

Muscatine County

Automatic Gravel Products Co., Box 34, Muscatine, Iowa.

Hahn Brothers Sand and Gravel Co., 207 W. Front Street, Muscatine, Iowa.

Northern Gravel Co., Muscatine, Iowa.

Plymouth County

Albert A. Wenzel, Kingsley, Iowa. 
Polk County

Builders Cooperative Sand Co., S. E. 3rd \& Jackson Avenue, Des Moines, Iowa.

Capital City Cooperative Sand \& Gravel Co., Box 864, Des Moines, Iowa.

The Des Moines Sand \& Fuel Coop. Ass'n., Box 1334, Des Moines, Iowa.

Flint Crushed Gravel Co., 907 Bankers Trust Building, Des Moines, lowa. Pit at West Des Moines.

N. Leon Harris, R. R. No. 4, Des Moines, Iowa.

Keefner Sand \& Gravel Co., 822 W. Ninth Street, Des Moines, Iowa.

Sac County

Wm. Brauer, R. F. D. No. 1, Lake View, Iowa.

Lake View Concrete Tile Co., Lake View, Iowa.

Northwestern Gravel Co., Lake View, Iowa.

LeGrand Limestone Co., 105 W. Madison Street, Chicago, Illinois. Quarry at Lake View.

W. H. Schnirring, Sac City, Iowa.

Mrs. W. H. Townsend, Sac City, Iowa.

Scott County

Builders Sand \& Gravel Co., 104 Western Avenue, Davenport, Iowa.

Sioux County

L. G. Everist, Inc., 2100 Fourth Street, Sioux City, Iowa.

Hawarden Gravel Co., Hawarden, Iowa.

Story County

R. E. Carr Sand \& Gravel Co., E. 16th Street, Ames, Iowa.

Roy Templeton, Ames, Iowa.

Tama County

Flint Crushed Gravel Co., 907 Bankers Trust Building, Des Moines, Iowa.

Van Buren County

J. C. Edvenson, Fort Dodge, Iowa. Pit at Stratford.

Wapello County

Ottumwa Sand Co., Ottumwa, Iowa.

Webster County

James Casey, Lehigh, Iowa.

Johnston Clay Works, Inc., Fort Dodge, Iowa. 
Earl Richardson, Stratford, Iowa.

James B. Weaver, National Bank \& Trust Co., Des Moines, Iowa.

Welch Bros., Fort Dodge, Iowa.

Winneshiek County

Decorah Concrete Products Co., 906 South Mill Street, Decorah, Iowa.

Wright County.

Luick Gravel Co., Belmond, Iowa.

A. A. McCurry, Renwick. Iowa.

Mrs. Etta Middleton, Eagle Grove, Iowa.

Miscellaneous Stone Producers in Iowa

\section{Commercial}

Harrison County

M. O. Weaver, Inc., 539 Fifth Street, Des Moines, Iowa.

Marshall County

Noncommercial

City of Des Moines Highway Department, Des Moines, Iowa.

\section{Peat Producers in Iowa}

Story County

Noncommercial

Iowa State Highway Commission, John M. Hall, Roadside Improvement Engineer, Ames, Iowa.

Worth County

Commercial

Colby Pioneer Peat Co., Inc., Hanlontown, Iowa.

Sandstone Producers in Iowa

Lucas County

Commercial

W. T. Kelly, Des Moines, Iowa.

Marion County

Noncommercial

Marion County Highway Department, Knoxville, Iowa.

\section{Lime Producers in Iowa}

Marion County

A. K. Verrifs, Pella, Iowa. 
